Supplementary Information

\title{
Diindenopyrenes: Extended 1,6- and 1,8-Pyrenoquinodimethanes with Singlet Diradical Characters
}

\author{
Daijiro Hibi, Kenichi Kitabayashi, Kazuya Fujita, Takashi Takeda, ${ }^{\dagger}$ Yoshito Tobe* \\ Division of Frontier Materials Science, Graduate School of Engineering Science, \\ Osaka University, 1-3 Machikaneyama, Toyonaka 560-8531, Japan \\ Tel.: +81-6-6850-6225; fax: +81-6-6850-6229 \\ ${ }^{\dagger}$ Present address: Institute of Multidisciplinary Research for Advanced Materials, Tohoku University, Sendai, \\ Miyagi, 980-8577, Japan.
}

page

1. X-Ray Crystallographic Analysis of 8b

Crystal Data (Table S1) and ORTEP Drawing for 8b (Figure S1)

2. Partial ${ }^{1} \mathrm{H}$ NMR Spectra for Aromatic Region with Theoretical and Experimental ${ }^{1} \mathrm{H}$ NMR Chemical Shifts of $3 b$ and $4 b$ (Figure S2), and $8 b$ and $9 b$ (Figure S3)

3. Theoretical and Experimental ${ }^{1} \mathrm{H}$ NMR Chemical Shifts and NOESY Spectrum of 11 (Figure S4)

4. Cyclic Voltammograms of $3 b$ and $4 b$ (Figure S5), and $8 b$ and $9 b$ (Figure S6)

5. Computational Results for 1a, 2a, 3a,b, 4a,b, 8a,b, 9a,b, 13a and 14a

S6

Calculated Energies and Spin-Squared Expectation Values $\left\langle\mathrm{S}^{2}>\right.$ calculated by RB3LYP/631(d) and UB3LYP/6-31G(d) methods (Table S2), HOMOs/LUMOs of 1a and 2a (Figure S7), 3a and 4a (Figure S8), 3b and 4b (Figure S9), 8a and 9a (Figure S10), 8b and 9b (Figure S11), 13a and 14a (Figure S12), and Spin Density Distributions for 1a, 2a, 3a, and 4a (Figure S13)

6. TD-DFT Calculations for 3b (Table S3), 4b (Table S4), 8b (Table S5), and 9b (Table S6)

S11

7. NMR Spectra of 1,4-Diiodo-5,8-bis(mesitylethynyl)naphthalene, 3b, 4b, 5b, 6c, 7, 8b, 9b, 11, and 13 (Figures S14-S23)

8. Cartesian Coordinates for Theoretically Optimized Geometries for 1a, 2a, 3a, 3b, 4a, 4b, 8a, 8b, 9a, 9b, 13a, 14a, 11, 16a, 16b, 16c, 16a', 16b', 17a, 17b, 18, 18', 19, 19', and 20' 


\section{X-Ray Crystallographic Analysis of $8 \mathrm{~b}$}

A single crystal of $\mathbf{8 b}$ suitable for X-ray diffraction study was obtained by recrystallization from $\mathrm{CH}_{2} \mathrm{Cl}_{2} / \mathrm{CH}_{3} \mathrm{CN}$. The data collection was performed with graphite monochromated MoK $\dot{\sim}$ radiation. The positional and thermal parameters of non-hydrogen atoms were refined anisotropically on $F 2$ by the full-matrix least-squares method using SHELXL-97. ${ }^{1}$ Hydrogen atoms were placed at calculated positions and refined "riding" on their corresponding carbon atoms. In the subsequent refinement, the function $\widetilde{\mathrm{w}}\left(F_{\mathrm{o}}{ }^{2}-F_{\mathrm{c}}{ }^{2}\right)^{2}$ was minimized, where $\left|F_{\mathrm{o}}\right|$ and $|F \mathrm{c}|$ are the observed and calculated structure factor amplitudes, respectively. The agreement indices are defined as $R 1=\tilde{\mathbf{\jmath}}(|| F \mathrm{o}|-| F \mathrm{c} \|) / \widetilde{\mathbf{\jmath}}|F \mathrm{o}|$ and $\mathrm{w} R 2=\left[\tilde{\mathrm{\jmath}} \mathrm{w}\left(F_{\mathrm{o}^{2}}-F_{\mathrm{c}}\right)^{2} / \widetilde{\jmath}\left(\mathrm{w} F \mathrm{o}^{2}\right)^{2}\right]^{1 / 2}$. The cystal data are summarized in Table S1.

(1) Programs for Crystal Structure Analysis (Release 97-2). Sheldrick, G. M.; Institut für Anorganische Chemie der Universität, Tammanstrasse 4, D-3400 Götingen, Germany, 1998.

Table S1. Crystal Data of $\mathbf{8 b}$.

\begin{tabular}{llll}
\hline formula & $\mathrm{C}_{54} \mathrm{H}_{47} \mathrm{I} \cdot \mathrm{CH}_{2} \mathrm{Cl}_{2}$ & $\alpha /$ deg. & $78.849(3)$ \\
$M$ & 1815.48 & $\therefore /$ deg. & $77.882(2)$ \\
$T / \mathrm{K}$ & 113 & $\circ / \mathrm{deg}$. & $64.901(2)$ \\
color & black & $V / \AA^{3}$ & $4235.3(6)$ \\
crystal system & triclinic & $Z$ & 2 \\
space group & $P-1$ bar & $D_{\mathrm{x}} / \mathrm{g} \mathrm{cm}^{-3}$ & 1.424 \\
$a / \AA$ & $16.0024(12)$ & No. of reflections & 18981 \\
$b / \AA$ & $16.2752(14)$ & $\mathrm{GOF}$ on $F^{2}$ & 0.983 \\
$c / \AA$ & $18.4727(16)$ & $R_{1}[I>2 \sigma(I)]$ & 0.0876 \\
& & $\mathrm{w} R_{2}$ (all data) & 0.2438 \\
\hline
\end{tabular}

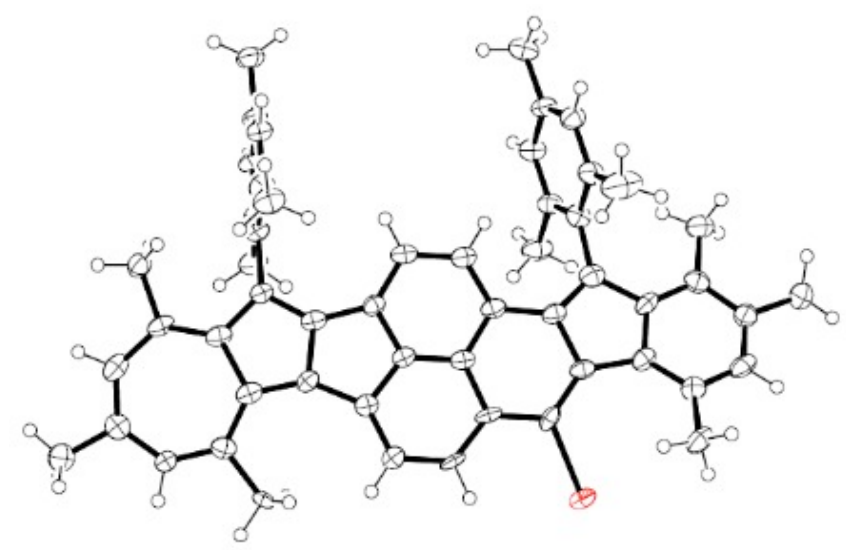

Figure S1. ORTEP drawing of $\mathbf{8 b}$. Displacement ellipsoids are drawn at 50\% probability level. 


\section{Theoretical and Experimental ${ }^{1} \mathrm{H}$ NMR Chemical Shifts of $3 \mathrm{~b}, 4 \mathrm{~b}, 8 \mathrm{~b}$, and $9 \mathrm{~b}$}
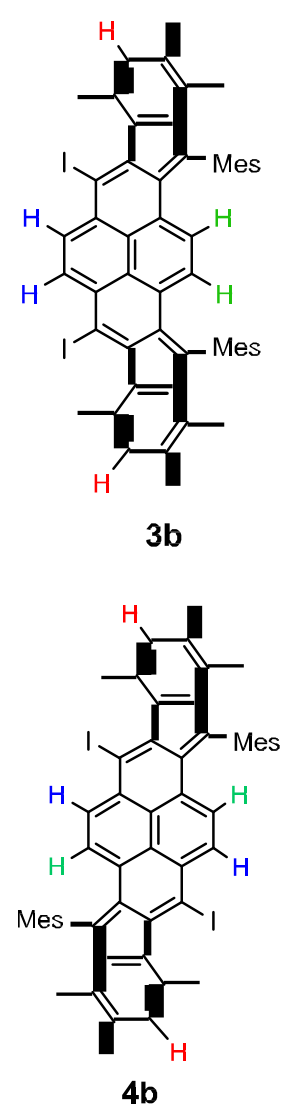
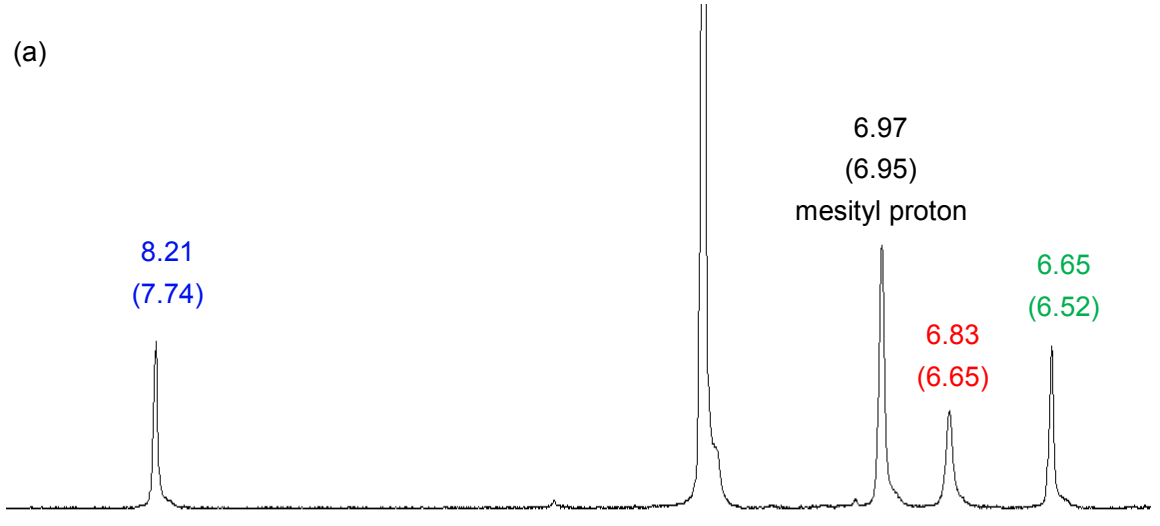

(b)
7.04

(7.08)

mesityl proton

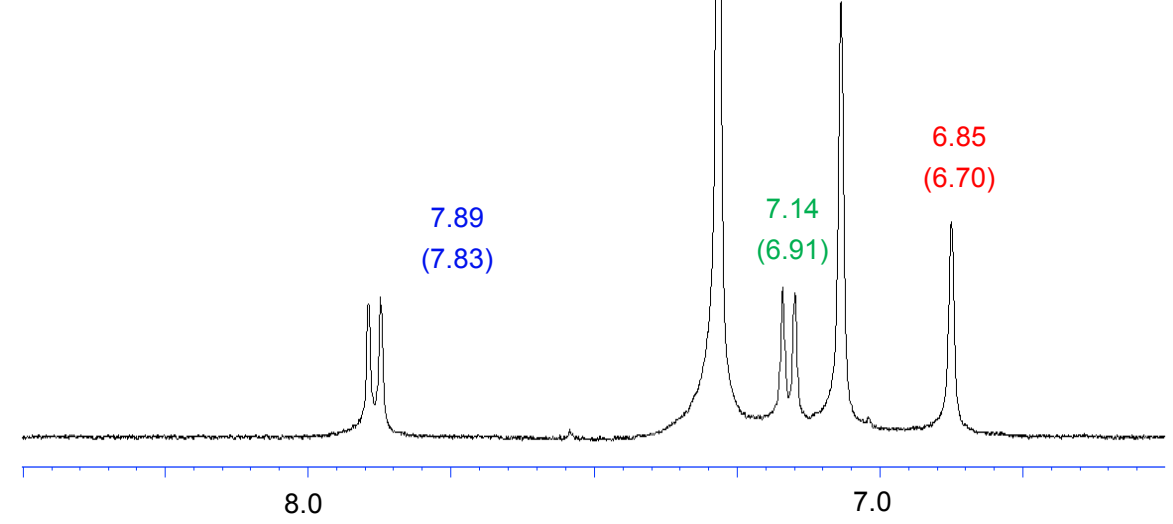

Figure S2. Partial ${ }^{1} \mathrm{H}$ NMR Spectra of (a) $\mathbf{3 b}$ and (b) $\mathbf{4 b}$ for Aromatic Region. The values indicate the experimental and theoretical chemical shifts (in parentheses). The partial assignments for the signals of naphthalene (blue and green) and benzene (red) protons are indicated by color codes. 


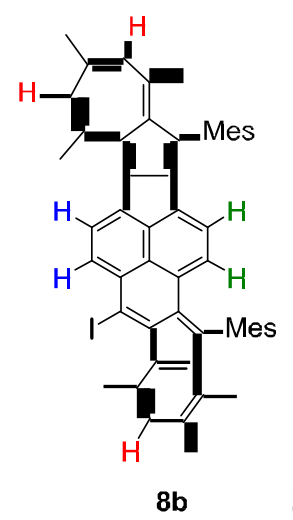

(a)
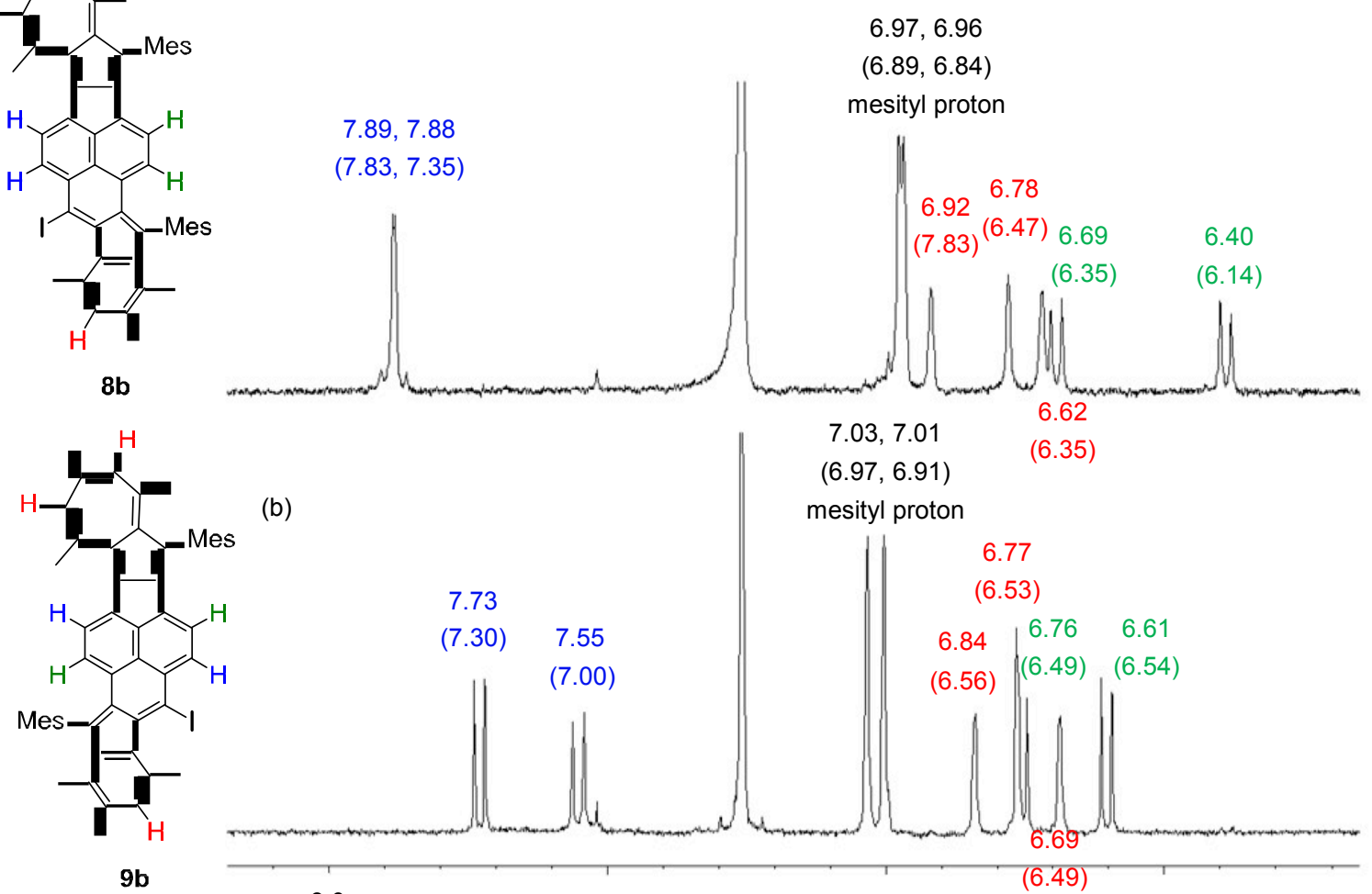

(b)

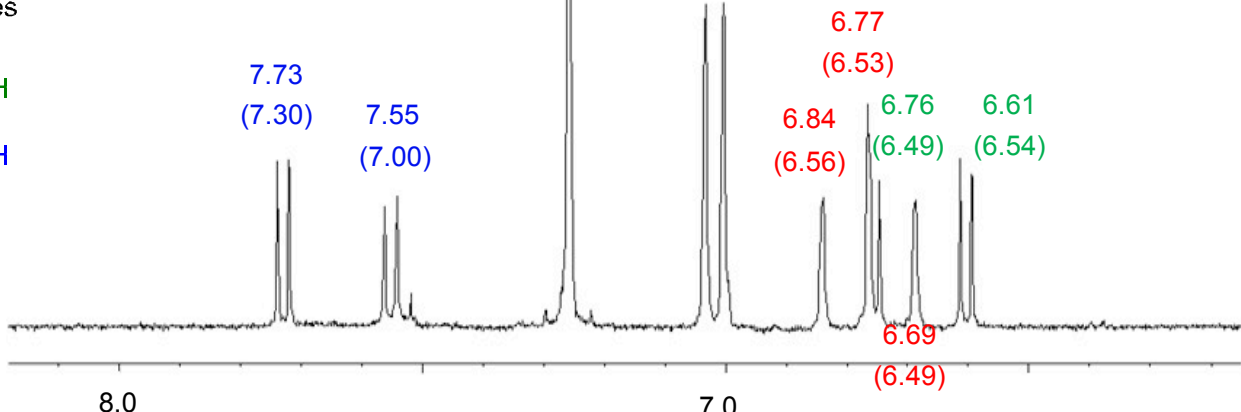

Figure S3. Partial ${ }^{1} \mathrm{H}$ NMR Spectra of (a) 8b and (b) 9b for Aromatic Region. The values indicate the experimental and theoretical chemical shifts (in parentheses). The partial assignments for the signals of naphthalene (blue and green), cycloheptatriene (red), and benzene (red) protons are indicated by color codes. 


\section{Theoretical and Experimental ${ }^{1} \mathrm{H}$ NMR Chemical Shifts and NOESY Spectrum of 11}
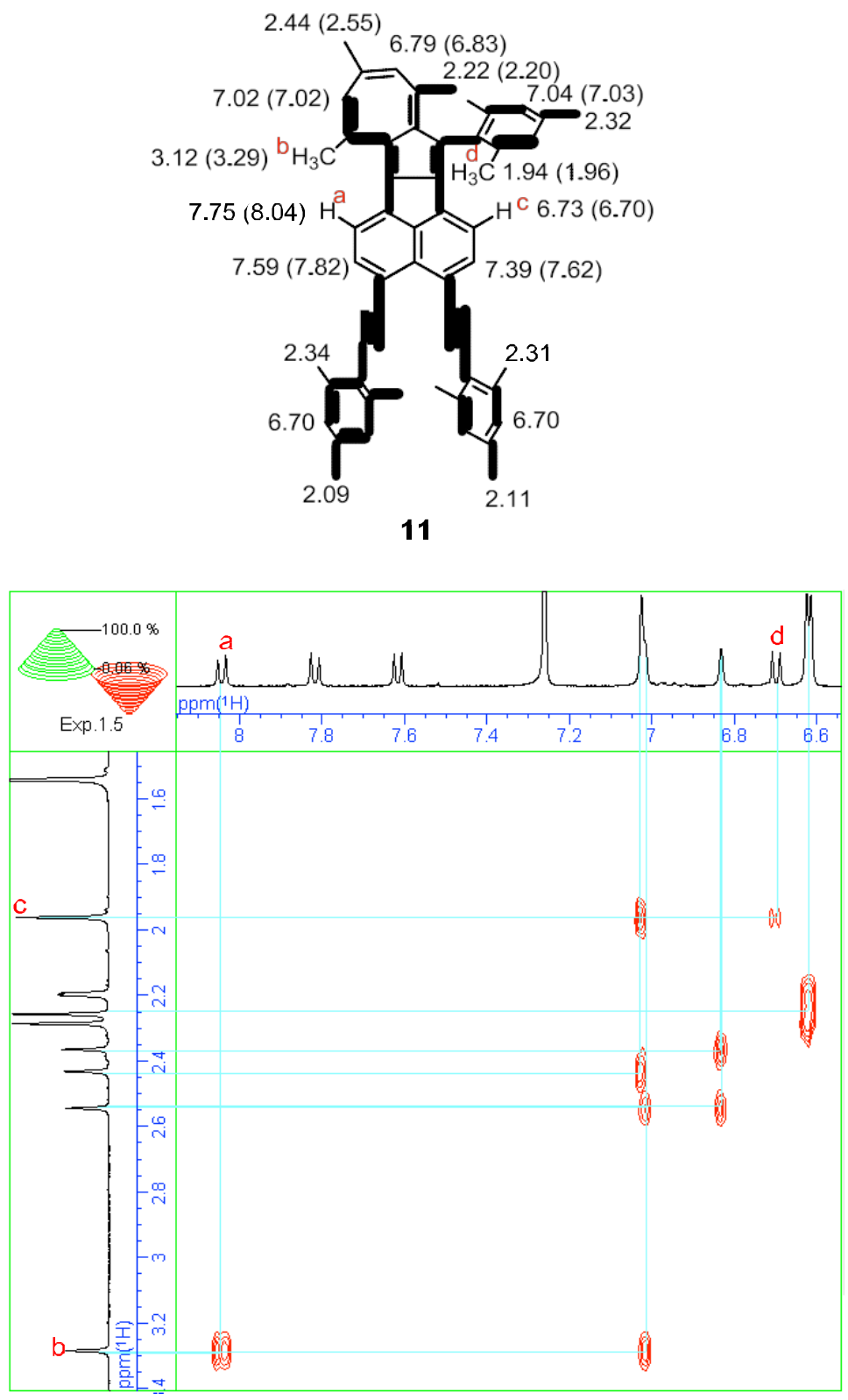

Figure S4. Partial NOESY Spectrum and Observed and Theoretical ${ }^{1} \mathrm{H}$ NMR Chemical Shifts of 11. Assignable observed chemical shifts are shown in parentheses. 


\section{Cyclic Voltammograms of $3 \mathrm{~b}, 4 \mathrm{~b}, 8 \mathrm{~b}$, and $9 \mathrm{~b}$}

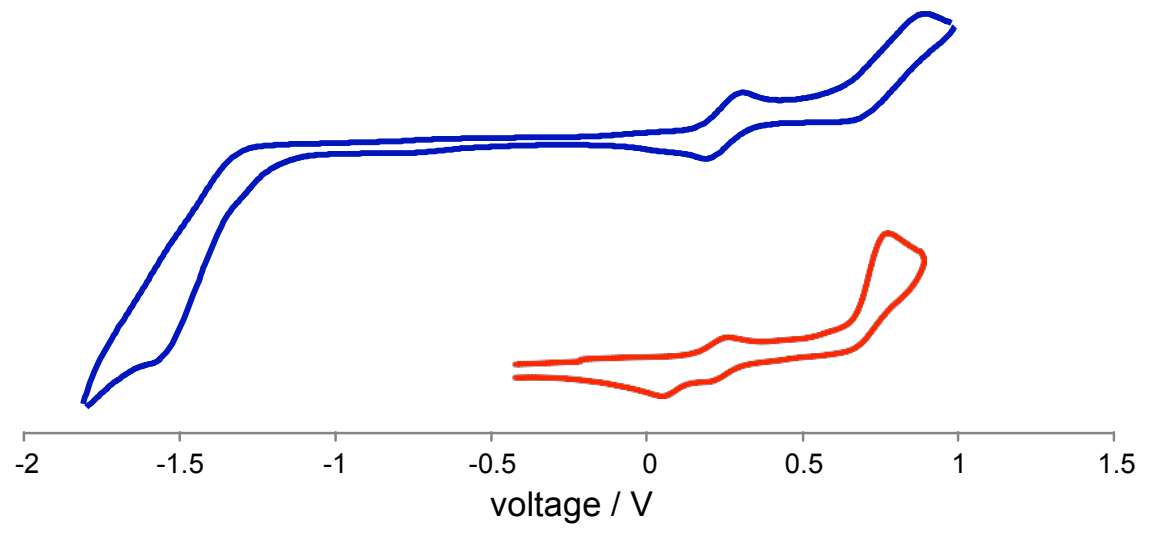

Figure S5. Cyclic Voltammograms of $\mathbf{3 b}$ (blue) and $\mathbf{4 b}$ (red) $(1 \mathrm{mM}$ and saturated $(<1 \mathrm{mM}$ because of low solubility) solutions of $\mathbf{3 b}$ and $\mathbf{4 b}$, respectively, in $\mathrm{CH}_{2} \mathrm{Cl}_{2}, \mathrm{~V}$ vs. $\mathrm{Fc} / \mathrm{Fc}^{+}$, containing $0.1 \mathrm{M}$ of $n \mathrm{Bu}_{4} \mathrm{NClO}_{4}$ as a supporting electrolyte, scan rate: $100 \mathrm{mV} / \mathrm{s}$ ). The red dotted voltammogram for $\mathbf{4 b}$ is for a scan at the first oxidation level, indicating the reduction wave at ca. $0.1 \mathrm{~V}$ is due to (an) oxidation product(s) generated by the second oxidation.

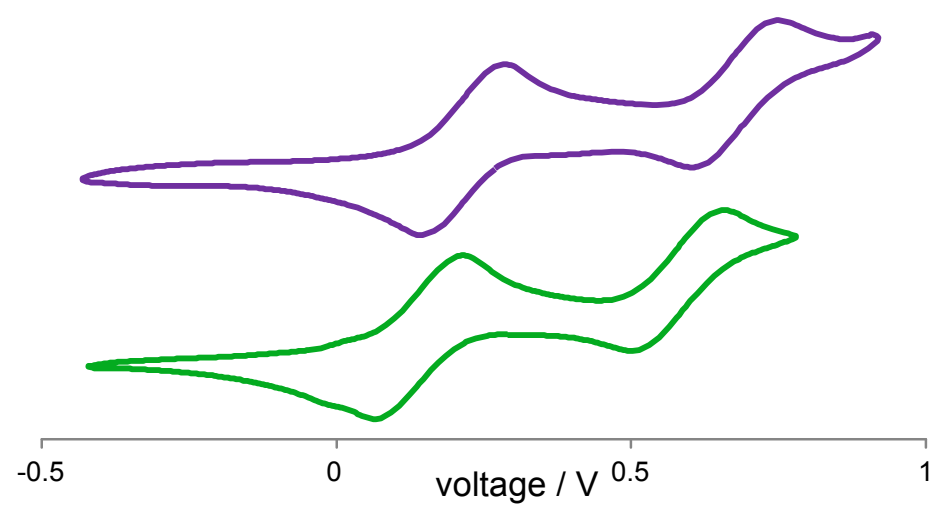

Figure S6. Cyclic Voltammograms of $\mathbf{8 b}$ (purple) and $\mathbf{9 b}$ (green) $\left(1 \mathrm{mM}\right.$ solution in $\mathrm{CH}_{2} \mathrm{Cl}_{2}, \mathrm{~V}$ vs. $\mathrm{Fc} / \mathrm{Fc}^{+}$, containing $0.1 \mathrm{M}$ of $n \mathrm{Bu}_{4} \mathrm{NClO}_{4}$ as a supporting electrolyte, scan rate: $100 \mathrm{mV} / \mathrm{s}$ ). 


\section{Computational Results for $1 \mathrm{a}, 2 \mathrm{a}, 3 \mathrm{3}, \mathrm{b}, 4 \mathrm{a}, \mathrm{b}, 8 \mathrm{a}, \mathrm{b}, 9 \mathrm{a}, \mathrm{b}, 13 \mathrm{a}$ and $14 \mathrm{a}$}

Table S2. Calculated Energies and Spin-Squared Expectation Values $<\mathrm{S}^{2}>$ for $\mathbf{1 a}, \mathbf{2 a}, \mathbf{3 a}, \mathbf{b}, \mathbf{4 a}, \mathbf{b}, \mathbf{8 a}, \mathbf{b}, 9 \mathbf{a}, \mathbf{b}$, 13a and 14a. ${ }^{a}$

\begin{tabular}{|c|c|c|c|c|c|}
\hline \multirow[b]{2}{*}{ Compd } & \multicolumn{2}{|c|}{ Singlet/RB3LYP/6-31G(d) } & \multicolumn{3}{|c|}{ Triplet/UB3LYP/6-31G(d) } \\
\hline & $\begin{array}{c}\text { Energy } \\
{[\text { hartree }]}\end{array}$ & $<\mathrm{S}^{2}>$ & $\begin{array}{c}\text { Energy } \\
{[\text { hartree ] }}\end{array}$ & Relative Energy & $<\mathrm{S}^{2}>$ \\
\hline $1 \mathbf{a}$ & -693.131932209 & 0 & -693.103423764 & $74.8 \mathrm{~kJ} / \mathrm{mol}$ & 2.0373 \\
\hline $2 a$ & -693.131182653 & 0 & -693.103210708 & $73.4 \mathrm{~kJ} / \mathrm{mol}$ & 2.0350 \\
\hline $3 \mathbf{a}$ & -1152.87229709 & 0 & -1152.85392463 & $48.2 \mathrm{~kJ} / \mathrm{mol}$ & 2.0355 \\
\hline $\mathbf{3 b}$ & -2108.24412708 & 0 & - & - & - \\
\hline $4 a$ & -1152.87244244 & 0 & -1152.85335772 & $50.1 \mathrm{~kJ} / \mathrm{mol}$ & 2.0356 \\
\hline $4 b$ & -2108.24412708 & 0 & - & - & - \\
\hline $\mathbf{8 a}$ & -1152.82541944 & 0 & - & - & - \\
\hline $8 b$ & -2097.43470388 & 0 & - & - & - \\
\hline $9 a$ & -1152.82452921 & 0 & - & - & - \\
\hline $9 \mathbf{b}$ & -2097.43392424 & 0 & - & - & - \\
\hline $13 a$ & -1152.76697336 & 0 & - & - & - \\
\hline $14 a$ & -1152.76800921 & 0 & - & - & - \\
\hline
\end{tabular}

${ }^{a}$ Calculated by the RB3LYP/6-31G(d) method for 1a, 2a, 3a, 4a, 8a, 9a, 13a, and 14a and the RB3LYP/6-31G(d) $(\mathrm{C}, \mathrm{H})+\mathrm{LanL} 2 \mathrm{DZ}$ (I) for $\mathbf{3 b}, \mathbf{4 b}, \mathbf{8 b}$, and $\mathbf{9 b}$. For $\mathbf{1 a}, \mathbf{2 a}, \mathbf{3 a}$, and $\mathbf{4 a}$, triplet energies were calculated by the UB3LYP/6-31G(d) method.

(a)

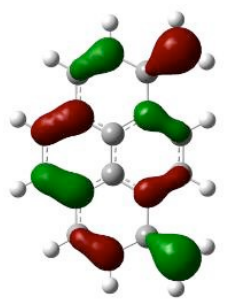

(b)

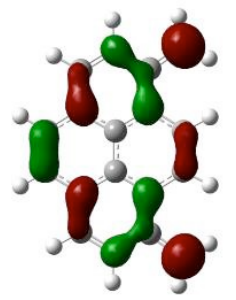

(c)

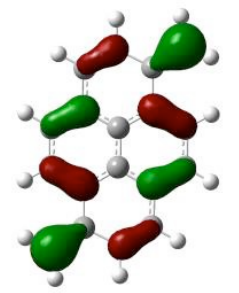

(d)

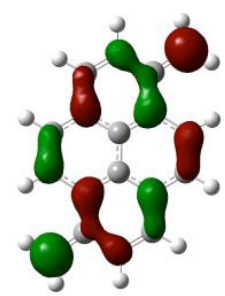

Figure S7. (a) HOMO and (b) LUMO of 1a and (c) HOMO and (d) LUMO of 2a 
(a)

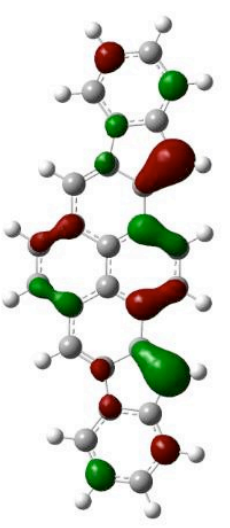

(b)

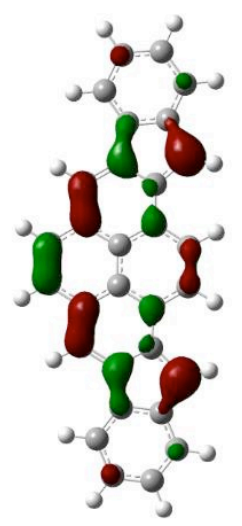

(c)

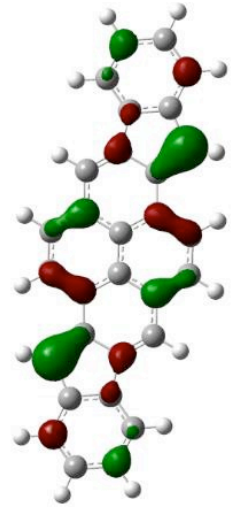

(d)

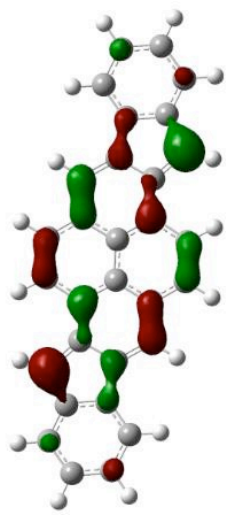

Figure S8. (a) HOMO and (b) LUMO of 3a and (c) HOMO and (d) LUMO of 4a

(a)

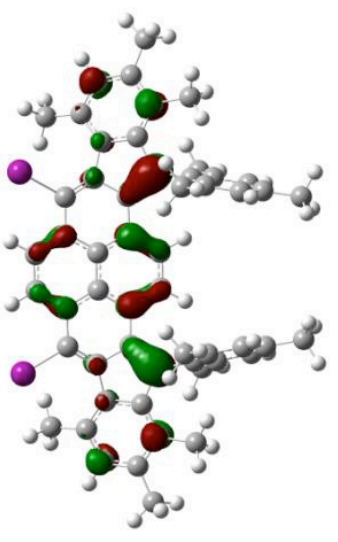

(b)

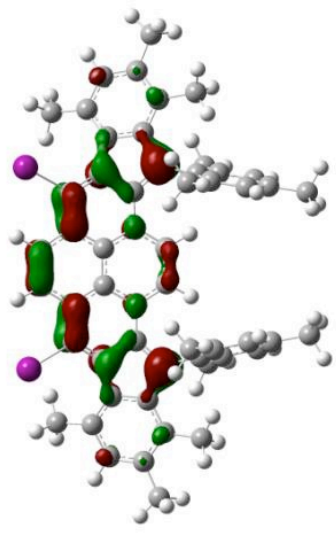

(c)

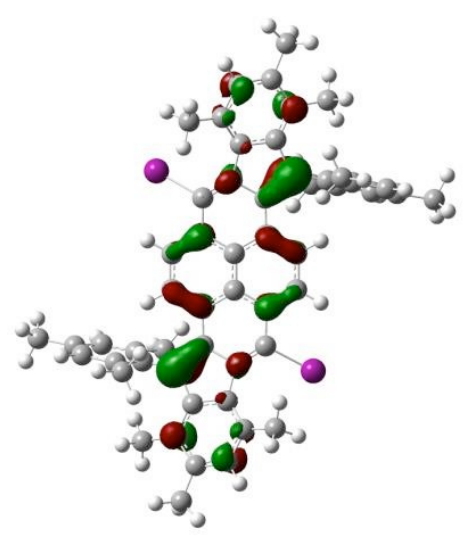

(d)

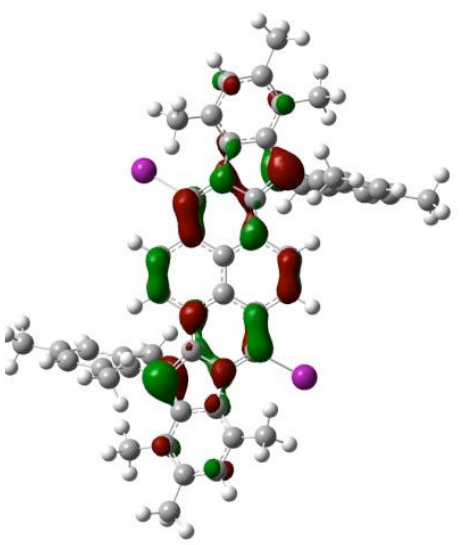

Figure S9. (a) HOMO and (b) LUMO of $\mathbf{3 b}$ and (c) HOMO and (d) LUMO of $\mathbf{4 b}$ 
(a)

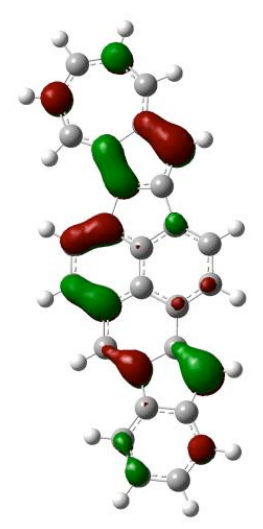

(b)

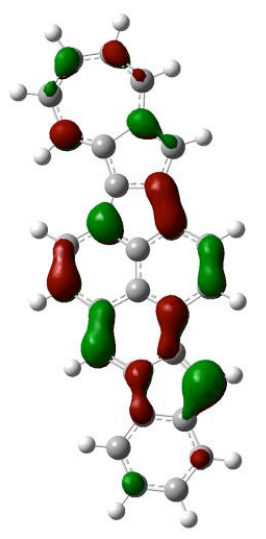

(c)

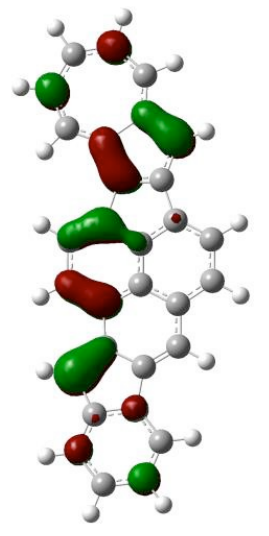

(d)

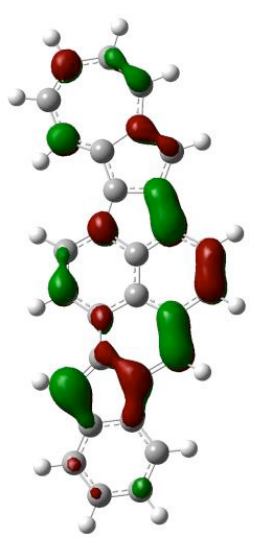

Figure S10. (a) HOMO and (b) LUMO of 8a and (c) HOMO and (d) LUMO of 9a

(a)

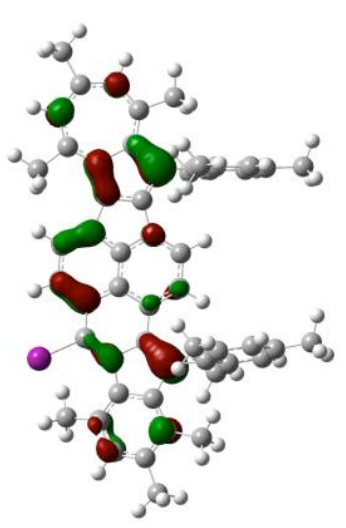

(b)
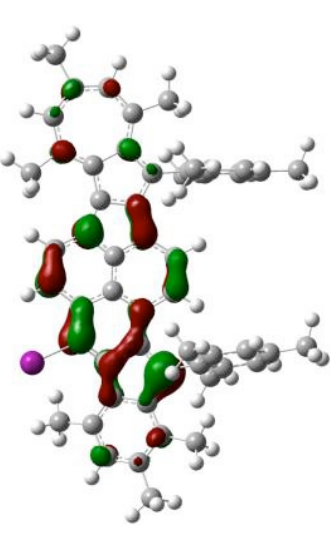

(c)

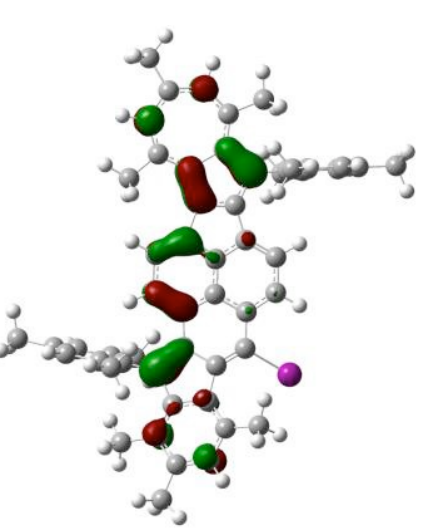

(d)

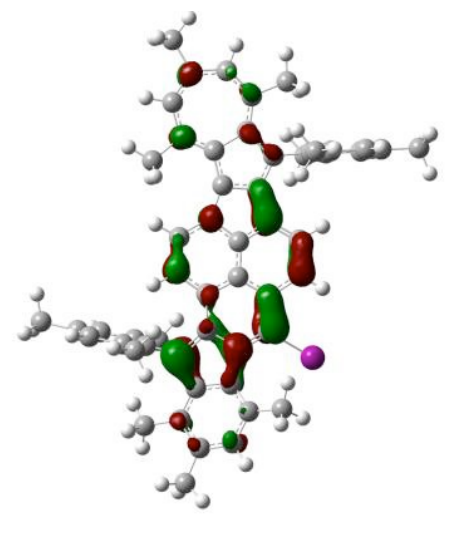

Figure S11. (a) HOMO and (b) LUMO of $\mathbf{8 b}$ and (c) HOMO and (d) LUMO of $\mathbf{9 b}$ 
(a)

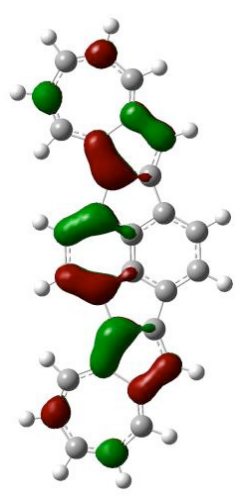

(b)

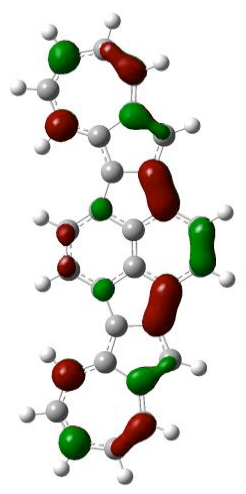

(c)

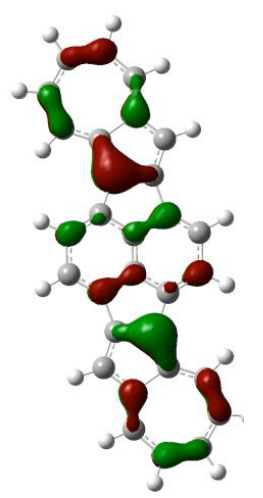

(d)

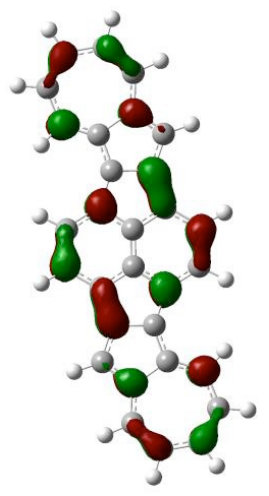

Figure S12. (a) HOMO and (b) LUMO of 13a and (c) HOMO and (d) LUMO of 14a

(a)

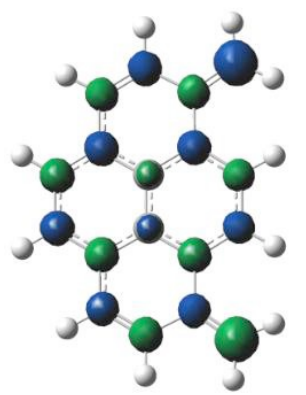

(b)

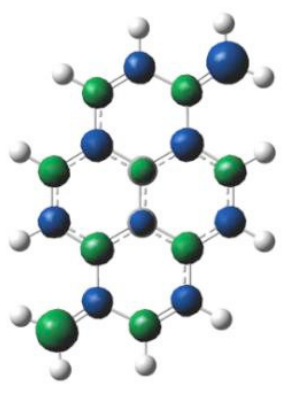

(c)

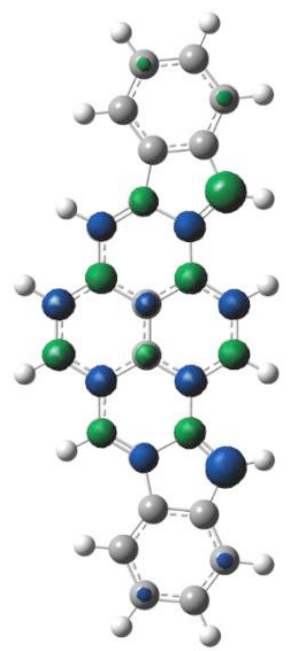

(d)

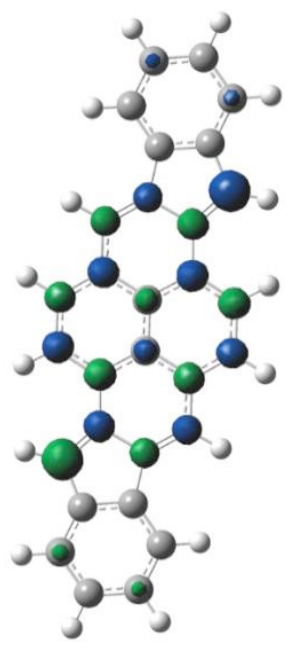

Figure S13. Spin Density Distributions of (a) 1a, (b) 2a, (c) 3a, and (d) 4a 


\section{TD-DFT Calculations for $3 b, 4 b, 8 b$, and $9 b$}

Table S3. Assignment of Electronic Transitions of $\mathbf{3 b}$ by TD-DFT Calculations. ${ }^{a}$

\begin{tabular}{|c|c|c|c|}
\hline Electronic Transition & $\begin{array}{c}\text { Transition Energy / eV } \\
\text { (Wavelength } / \mathrm{nm})\end{array}$ & Main CI Coefficient & $\begin{array}{c}\text { Oscillator Strength } \\
(f)\end{array}$ \\
\hline 1 & $1.63 \mathrm{eV}$ & $0.154(\mathrm{HOMO}-1-$ LUMO) & 0.676 \\
& $(759)$ & $0.688(\mathrm{HOMO}-\mathrm{LUMO})$ & \\
\hline 2 & $2.20 \mathrm{eV}$ & $0.686(\mathrm{HOMO}-1-\mathrm{LUMO})$ & 0.293 \\
& $(563)$ & $-0.152(\mathrm{HOMO}-\mathrm{LUMO})$ & 0.009 \\
\hline 3 & $2.43 \mathrm{eV}$ & $-0.256(\mathrm{HOMO}-3-\mathrm{LUMO})$ & 0.011 \\
& $(510)$ & $0.647(\mathrm{HOMO}-2-$ LUMO) & \\
\hline 4 & $2.63 \mathrm{eV}$ & $0.511(\mathrm{HOMO}-3-\mathrm{LUMO})$ & \\
& $(471)$ & $0.179(\mathrm{HOMO}-2-\mathrm{LUMO})$ & \\
\hline
\end{tabular}

$a$ RB3LYP/6-31G(d) (C, H) + LanL2DZ (I)

Table S4. Assignment of Electronic Transitions of $\mathbf{4 b}$ by TD-DFT Calculations. ${ }^{a}$

\begin{tabular}{|c|c|c|c|}
\hline Electronic Transition & $\begin{array}{c}\text { Transition Energy / eV } \\
\text { (Wavelength / nm) }\end{array}$ & Main CI Coefficient & $\begin{array}{c}\text { Oscillator Strength } \\
(f)\end{array}$ \\
\hline 1 & $1.74 \mathrm{eV}$ & $0.704(\mathrm{HOMO}-\mathrm{LUMO})$ & 1.058 \\
\hline 2 & $2.09 \mathrm{eV}$ & $0.702(\mathrm{HOMO}-1-\mathrm{LUMO})$ & 0 \\
\hline 3 & $(593)$ & $-0.425(\mathrm{HOMO}-2-\mathrm{LUMO})$ & 0 \\
\hline 4 & $2.52 \mathrm{eV}$ & $0.559(\mathrm{HOMO}-\mathrm{LUMO}+1)$ & 0.003 \\
& $(492)$ & $0.540(\mathrm{HOMO}-2-\mathrm{LUMO})$ & \\
\hline & $2.59 \mathrm{eV}$ & $0.418(\mathrm{HOMO}-\mathrm{LUMO}+1)$ & \\
\hline & $(478)$ & $0.107(\mathrm{HOMO}-$ LUMO+3) & \\
\hline
\end{tabular}

$a$ RB3LYP/6-31G(d) (C, H) + LanL2DZ (I) 
Table S5. Assignment of Electronic Transitions of $\mathbf{8 b}$ by TD-DFT Calculations. ${ }^{a}$

\begin{tabular}{|c|c|c|c|}
\hline $\begin{array}{l}\text { Electronic } \\
\text { Transition }\end{array}$ & $\begin{array}{c}\text { Transition Energy / eV } \\
\text { (Wavelength / nm) }\end{array}$ & Main CI Coefficient & Oscillator Strength $(f)$ \\
\hline 1 & $\begin{array}{c}1.85 \mathrm{eV} \\
(668)\end{array}$ & $\begin{array}{c}-0.327 \text { (HOMO-1 - LUMO) } \\
0.111(\mathrm{HOMO}-1 \text { - LUMO+1) } \\
0.606(\mathrm{HOMO}-\text { LUMO) }\end{array}$ & 0.450 \\
\hline 2 & $\begin{array}{c}1.99 \mathrm{eV} \\
(624)\end{array}$ & $\begin{array}{c}0.617(\mathrm{HOMO}-1 \text { - LUMO) } \\
0.303(\mathrm{HOMO}-\mathrm{LUMO})\end{array}$ & 0.261 \\
\hline 3 & $\begin{array}{c}2.30 \mathrm{eV} \\
(540)\end{array}$ & $\begin{array}{c}-0.106(\mathrm{HOMO}-1-\mathrm{LUMO}+1) \\
0.147(\mathrm{HOMO}-\mathrm{LUMO}) \\
0.664(\mathrm{HOMO}-\text { LUMO+1) }\end{array}$ & 0.173 \\
\hline 4 & $\begin{array}{c}2.70 \mathrm{eV} \\
(459)\end{array}$ & $\begin{array}{c}0.601(\mathrm{HOMO}-2-\mathrm{LUMO}) \\
0.294(\mathrm{HOMO}-1-\mathrm{LUMO}+1) \\
0.148(\mathrm{HOMO}-\mathrm{LUMO}+1)\end{array}$ & 0.237 \\
\hline
\end{tabular}

$a$ RB3LYP/ 6-31G(d) (C, H) + LanL2DZ (I)

Table S6. Assignment of Electronic Transitions of $\mathbf{9 b}$ by TD-DFT Calculations. ${ }^{a}$

\begin{tabular}{|c|c|c|c|}
\hline Electronic Transition & $\begin{array}{c}\text { Transition Energy / eV } \\
\text { (Wavelength / nm) }\end{array}$ & Main CI Coefficient & Oscillator Strength $(f)$ \\
\hline 1 & $\begin{array}{c}1.69 \mathrm{eV} \\
(734)\end{array}$ & $\begin{array}{c}-0.243 \text { (HOMO-1 - LUMO) } \\
0.605 \text { (HOMO - LUMO) } \\
0.118 \text { (HOMO - LUMO+1) }\end{array}$ & 0.168 \\
\hline 2 & $\begin{array}{c}2.18 \mathrm{eV} \\
(569)\end{array}$ & $\begin{array}{l}0.152(\mathrm{HOMO}-2-\mathrm{LUMO}) \\
0.517(\mathrm{HOMO}-1-\mathrm{LUMO}) \\
0.398(\mathrm{HOMO}-\mathrm{LUMO}+1)\end{array}$ & 0.113 \\
\hline 3 & $\begin{array}{c}2.37 \mathrm{eV} \\
(524)\end{array}$ & $\begin{array}{c}0.275 \text { (HOMO-2- LUMO) } \\
-0.332(\mathrm{HOMO}-1 \text { - LUMO) } \\
-0.107(\mathrm{HOMO}-1 \text { - LUMO+1) } \\
-0.157(\mathrm{HOMO}-\mathrm{LUMO}) \\
0.402(\mathrm{HOMO}-\mathrm{LUMO}+1) \\
-0.156(\mathrm{HOMO}-\mathrm{LUMO}+2)\end{array}$ & 0.376 \\
\hline 4 & $\begin{array}{c}2.57 \mathrm{eV} \\
(483)\end{array}$ & $\begin{array}{c}0.135 \text { (HOMO-3- LUMO) } \\
0.570 \text { (HOMO-2 - LUMO) } \\
-0.119(\mathrm{HOMO}-1-\mathrm{LUMO}+1) \\
-0.319(\mathrm{HOMO}-\mathrm{LUMO}+1)\end{array}$ & 0.127 \\
\hline
\end{tabular}

$a$ RB3LYP/ 6-31G(d) (C, H) + LanL2DZ (I) 
7. NMR Spectra of 1,4-Diiodobis-5,8-bis(mesitylethynyl)naphthalene, 3b, 4b, 5b, 6c, 7, 8b, 9b, 11, and 15

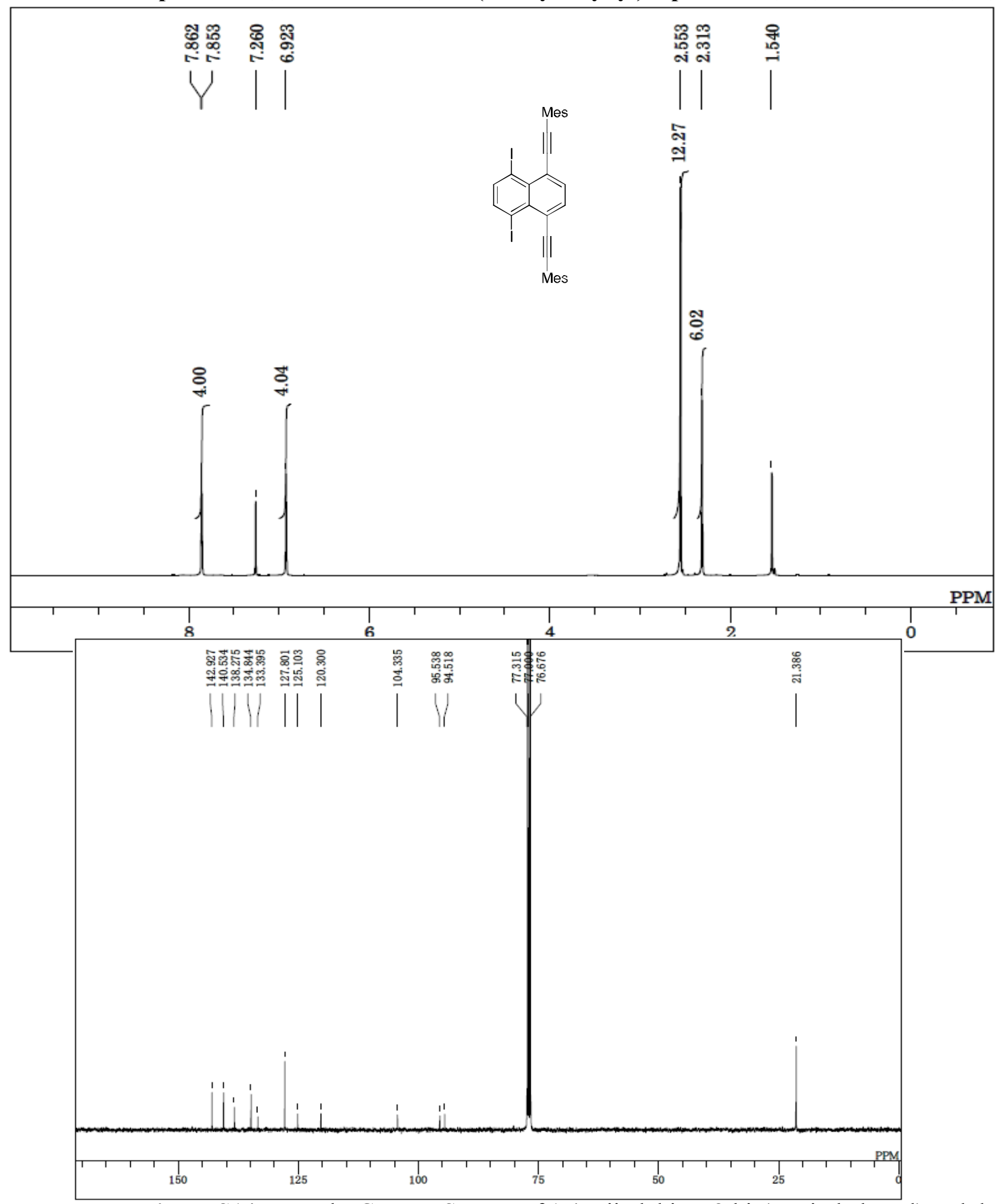

Figure S14. H and C NMR Spectra of 1,4-Diiodobis-5,8-bis(mesitylethynyl)naphthalene. 


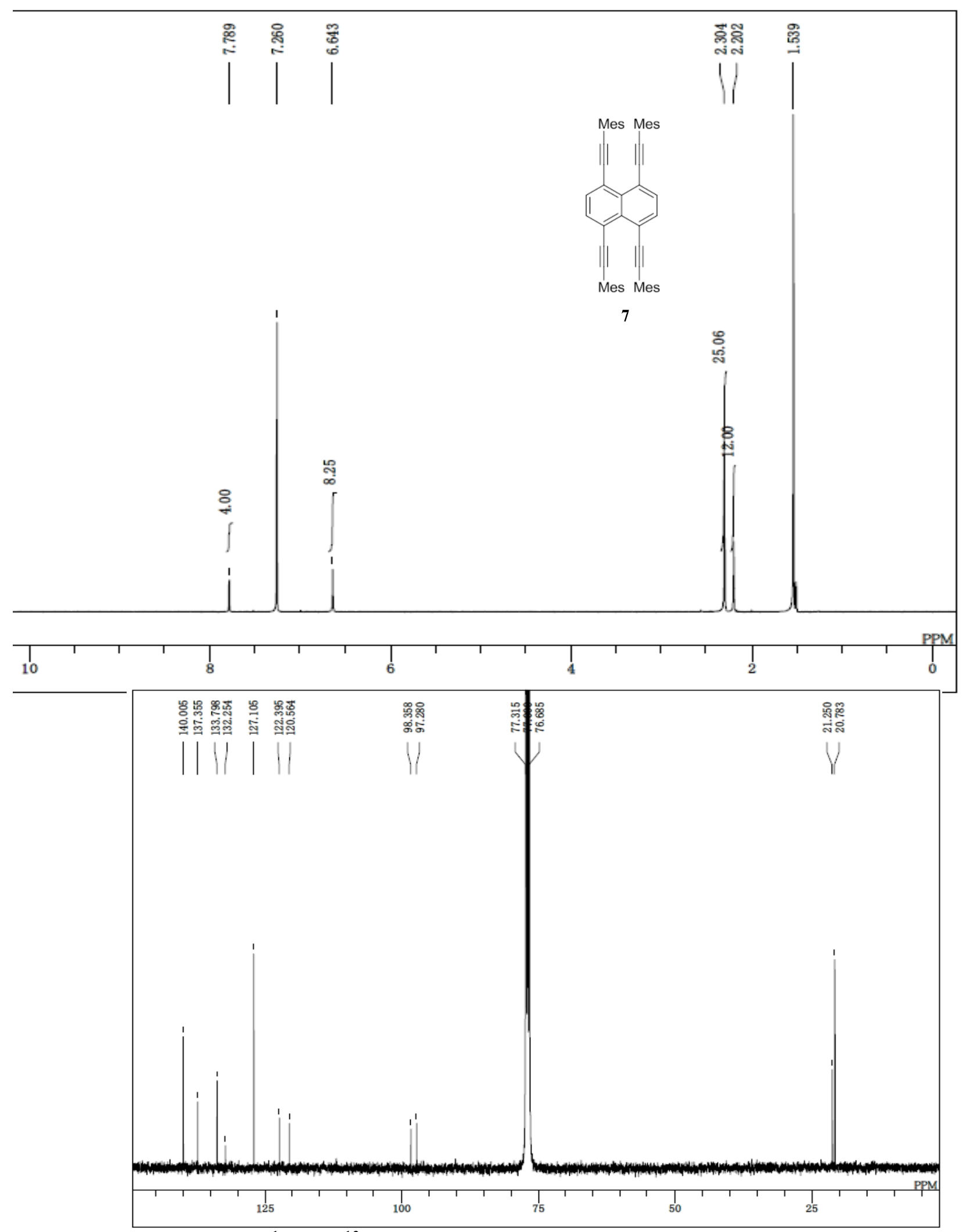

Figure S15. ${ }^{1} \mathrm{H}$ and ${ }^{13} \mathrm{C}$ NMR Spectra of Tetrakis(mesitylethynyl)naphthalene (7). 


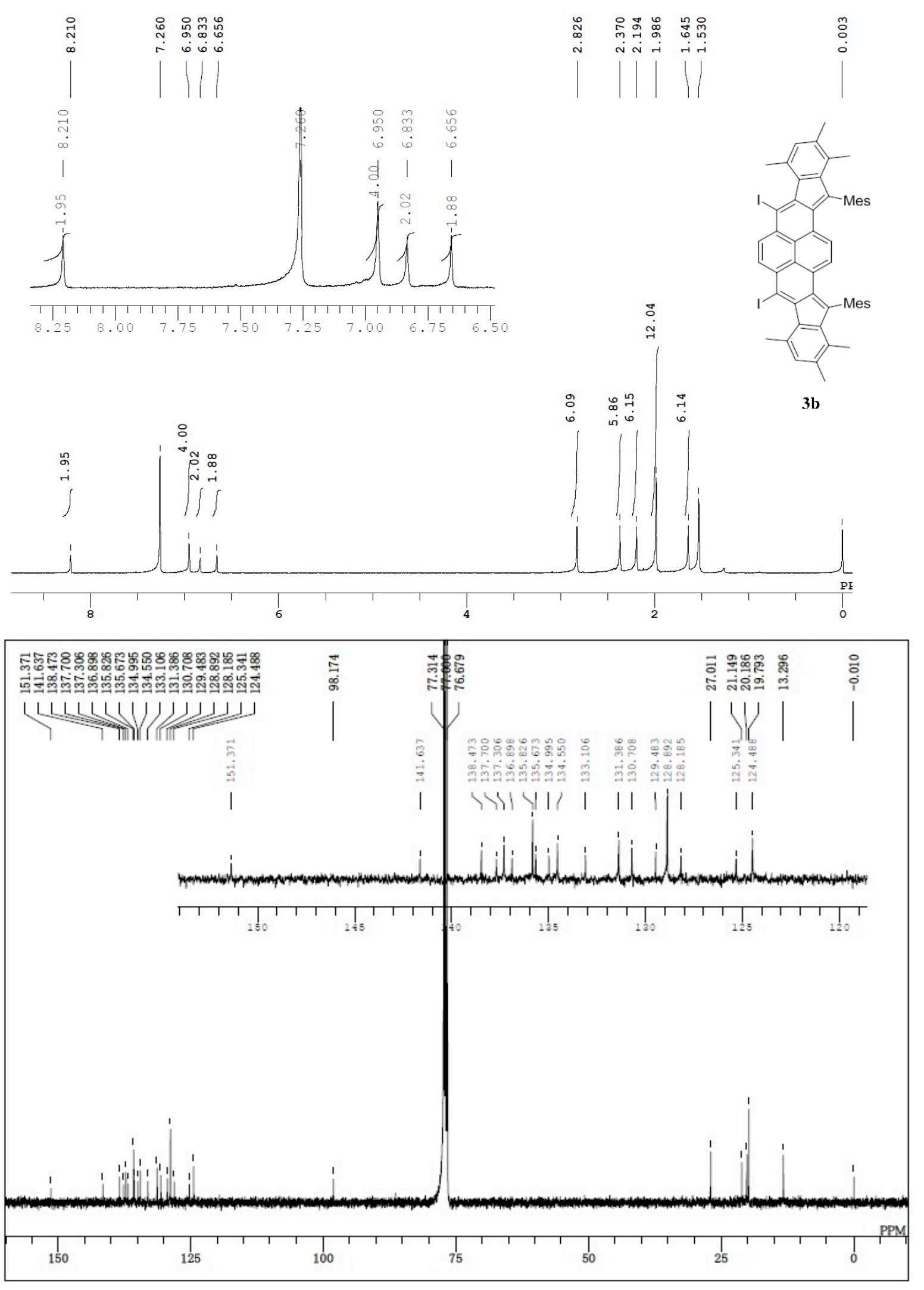

Figure S16. ${ }^{1} \mathrm{H}$ and ${ }^{13} \mathrm{C}$ NMR Spectra of syn-Diindenopyrene $\mathbf{3 b}$. 


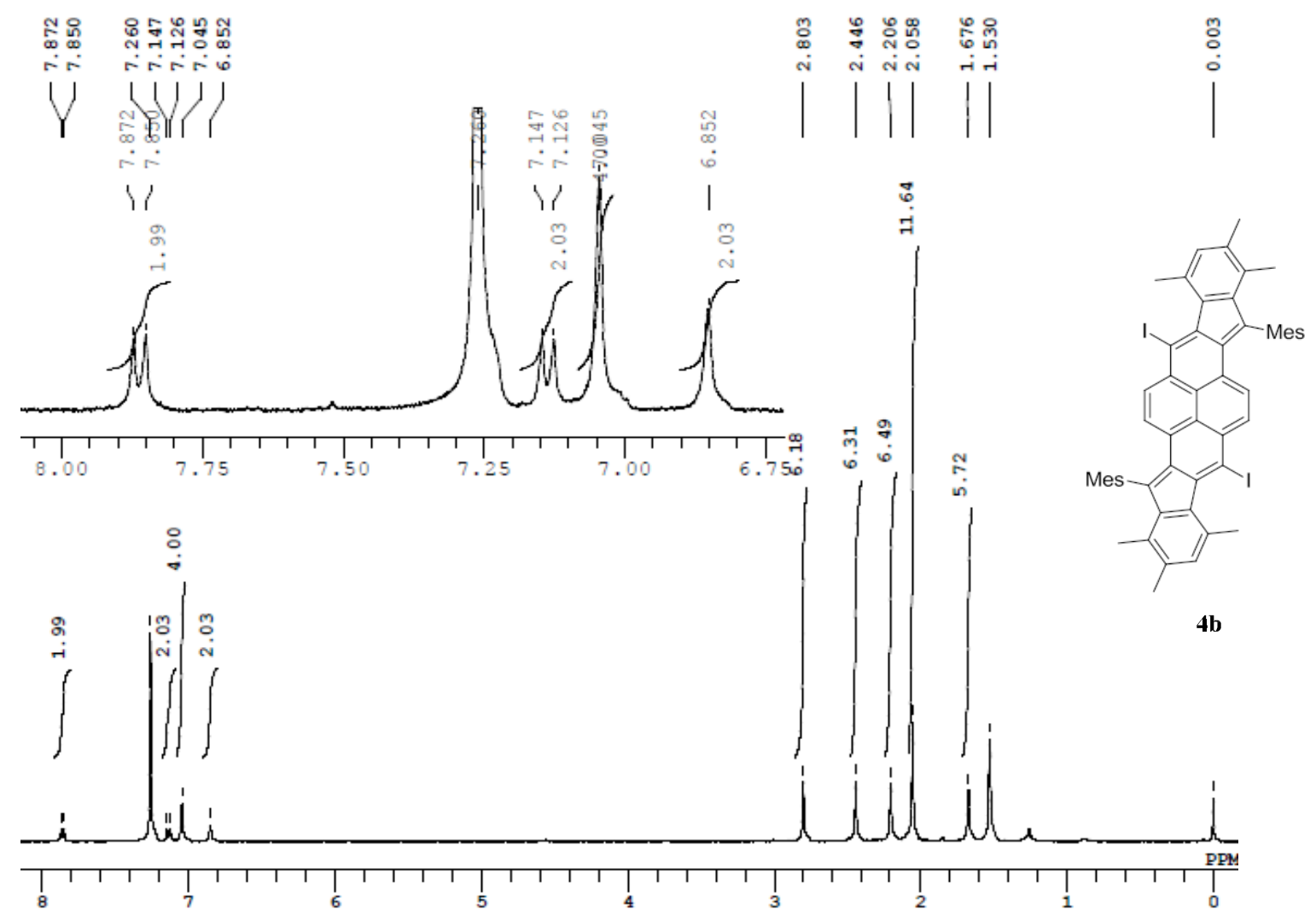

Figure S17. ${ }^{1} \mathrm{H}$ NMR Spectrum of anti-Diindenopyrene $\mathbf{4 b}$. 


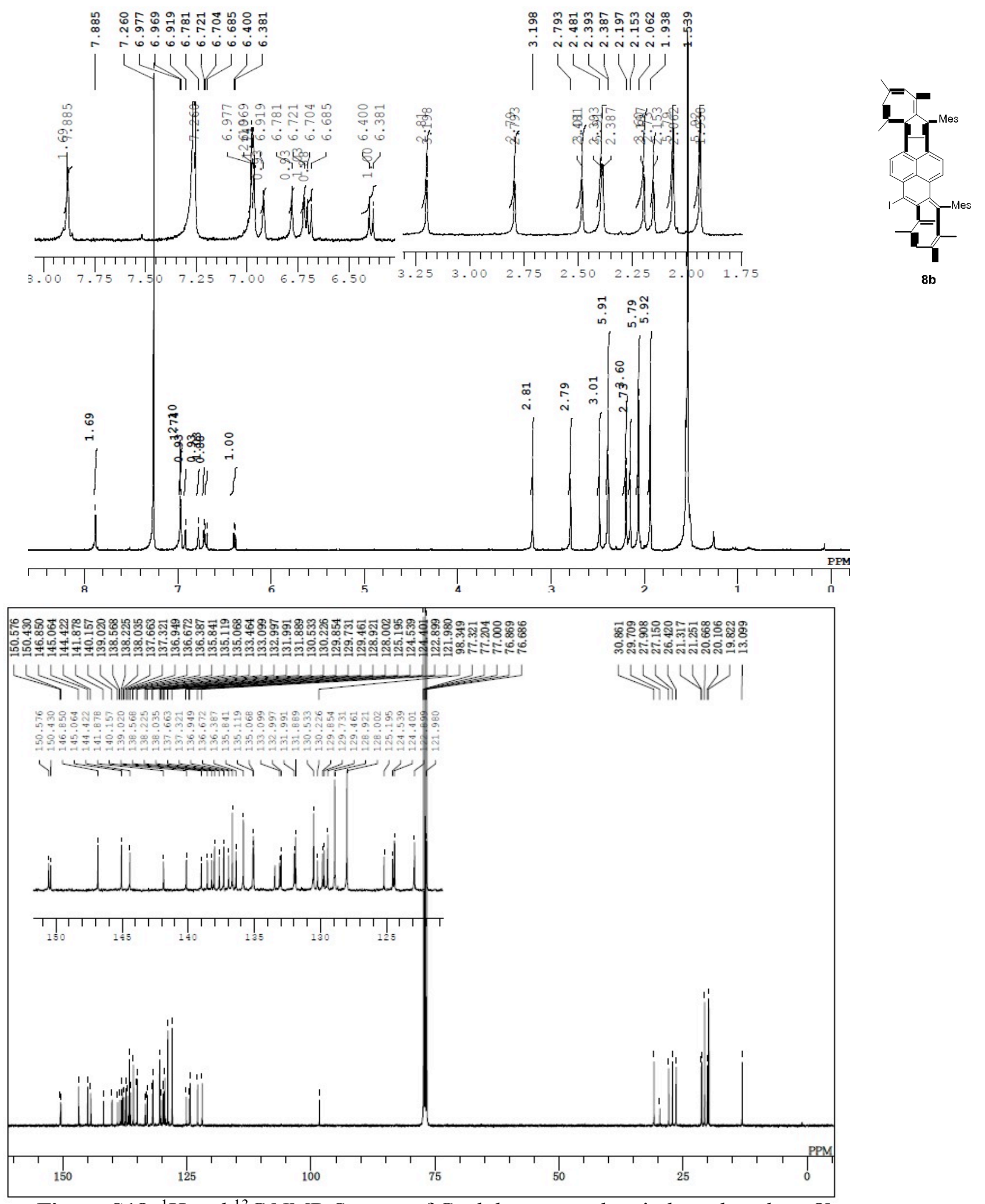

Figure S18. ${ }^{1} \mathrm{H}$ and ${ }^{13} \mathrm{C}$ NMR Spectra of Cycloheptapentalenoindenophenalene $\mathbf{8 b}$. 


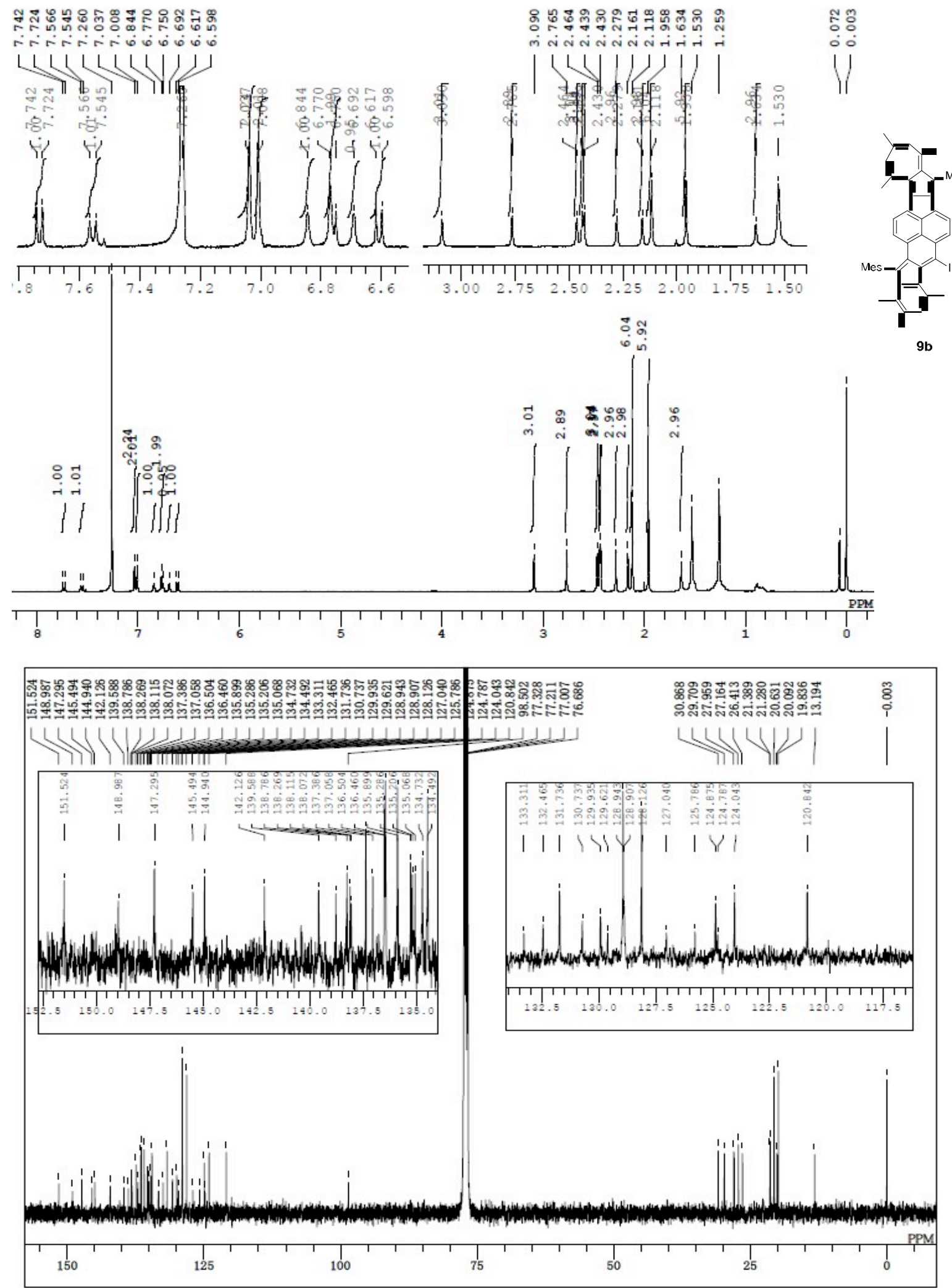

Figure S19. ${ }^{1} \mathrm{H}$ and ${ }^{13} \mathrm{C}$ NMR Spectra of Cycloheptapentalenoindenophenalene 9b. 


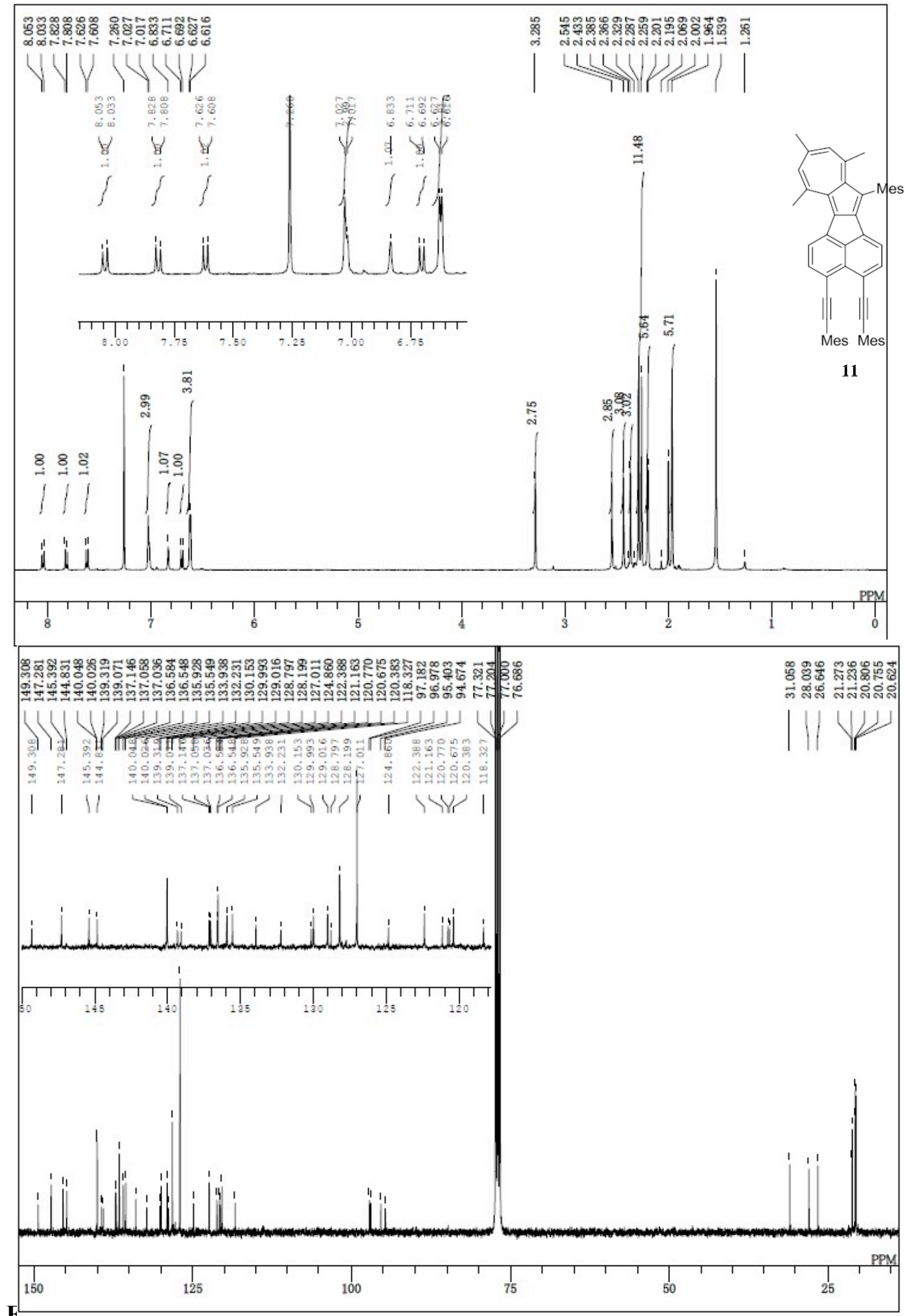




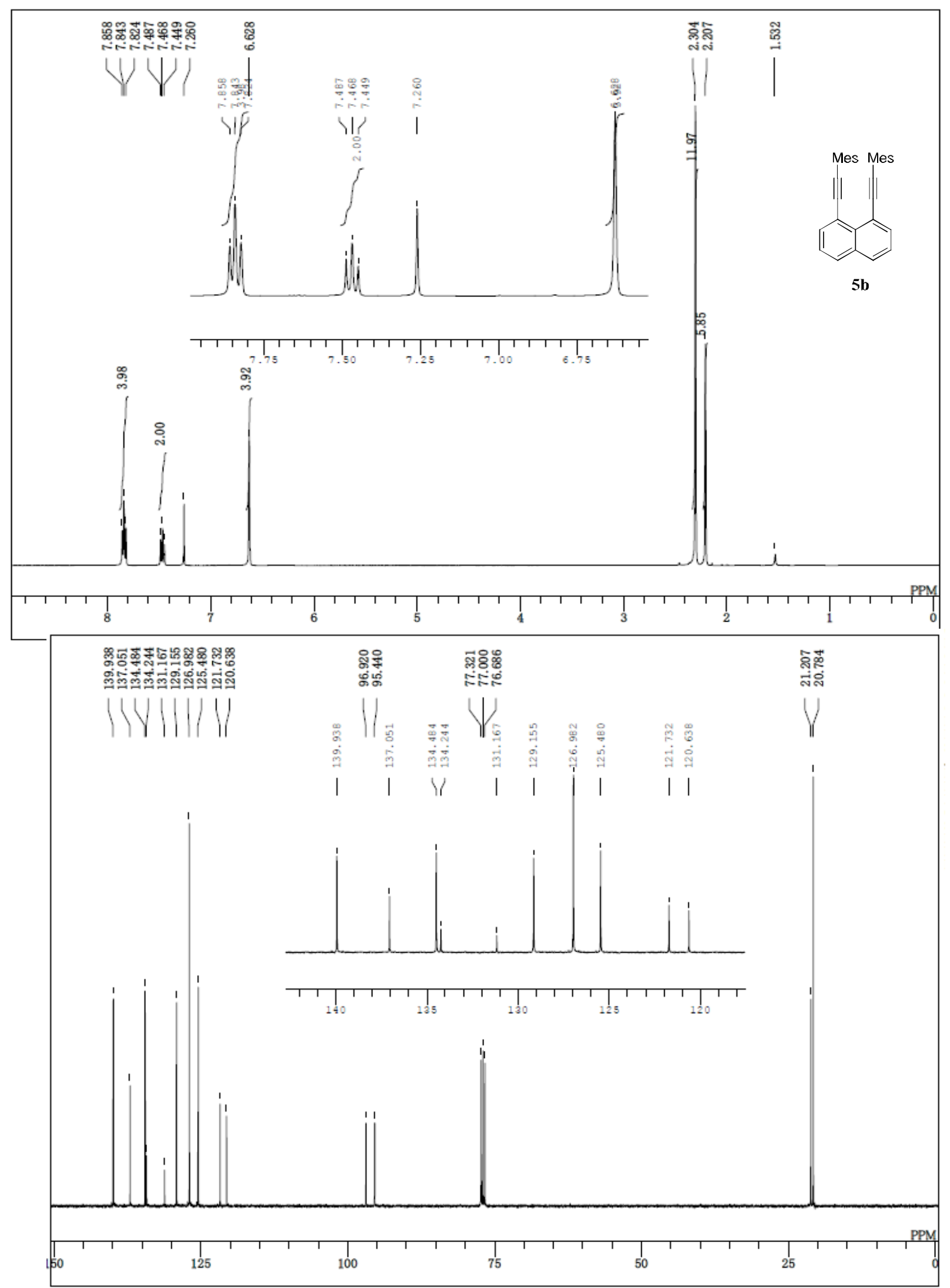

Figure S21. ${ }^{1} \mathrm{H}$ and ${ }^{13} \mathrm{C}$ NMR Spectra of Bis(mesitylethynyl)naphthalene (5b). 


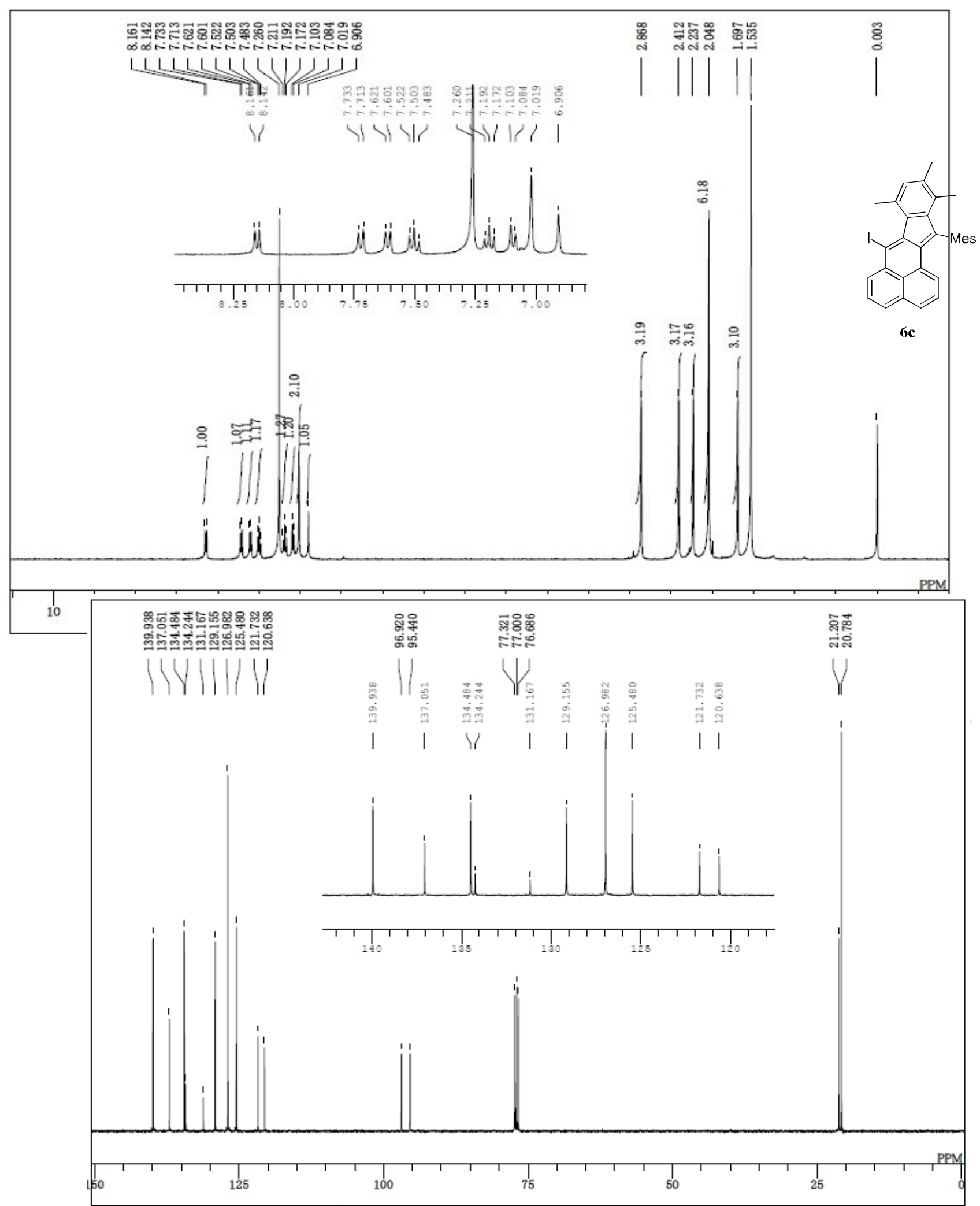

Figure S22. ${ }^{1} \mathrm{H}$ and ${ }^{13} \mathrm{C}$ NMR Spectra of Indenophenalene 6c. 


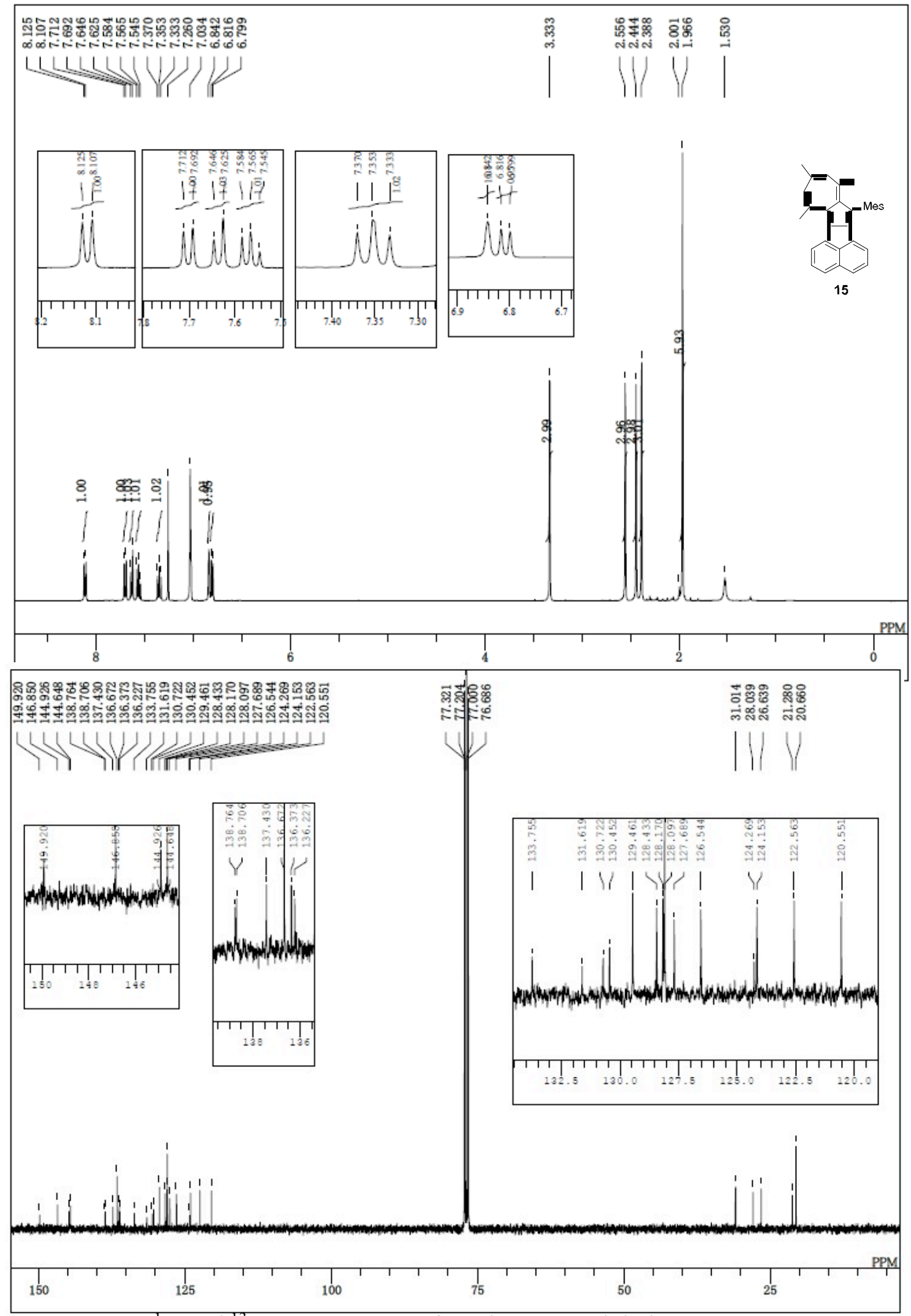

Figure S23. ${ }^{1} \mathrm{H}$ and ${ }^{13} \mathrm{C}$ NMR Spectra of Azulenoacenaphthylene 15. 


\section{Cartesian Coordinates for Theoretically Optimized Geometries}

\section{1,6-Pyrenoquinodimethane (1a)}

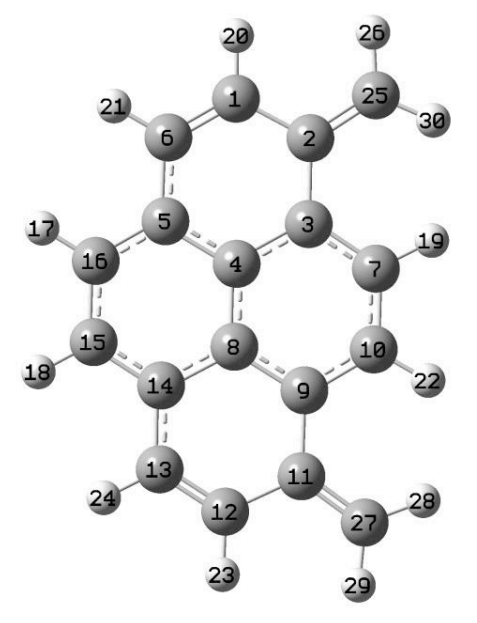

SCF Done: $\quad E(R B 3 L Y P)=-693.131932209$

A.U. after

\begin{tabular}{|c|c|c|c|c|}
\hline No. & Atom & $\mathrm{X}$ & $\mathrm{Y}$ & $\mathrm{Z}$ \\
\hline 1 & $\mathrm{C}$ & 3.564218 & 0.358925 & 0.000333 \\
\hline 2 & $\mathrm{C}$ & 2.918138 & -0.947677 & 0.000285 \\
\hline 3 & $\mathrm{C}$ & 1.435151 & -0.959273 & 0.000145 \\
\hline 4 & $\mathrm{C}$ & 0.714885 & 0.273055 & 0.000064 \\
\hline 5 & $\mathrm{C}$ & 1.417472 & 1.526486 & 0.000118 \\
\hline 6 & $\mathrm{C}$ & 2.863552 & 1.512976 & 0.000256 \\
\hline 7 & $\mathrm{C}$ & 0.696822 & -2.142575 & 0.000088 \\
\hline 8 & $\mathrm{C}$ & -0.714885 & 0.273055 & -0.000072 \\
\hline 9 & $\mathrm{C}$ & -1.435151 & -0.959273 & -0.000126 \\
\hline 10 & $\mathrm{C}$ & -0.696822 & -2.142575 & -0.000044 \\
\hline 11 & $\mathrm{C}$ & -2.918139 & -0.947677 & -0.000266 \\
\hline 12 & $\mathrm{C}$ & -3.564217 & 0.358925 & -0.000343 \\
\hline 13 & $\mathrm{C}$ & -2.863552 & 1.512976 & -0.000290 \\
\hline 14 & $\mathrm{C}$ & -1.417472 & 1.526486 & -0.000152 \\
\hline 15 & $\mathrm{C}$ & -0.700704 & 2.716465 & -0.000097 \\
\hline
\end{tabular}

7 cycles

\begin{tabular}{|c|c|c|c|c|}
\hline No. & Atom & $\mathrm{X}$ & $\mathrm{Y}$ & $\mathrm{Z}$ \\
\hline 16 & $\mathrm{C}$ & 0.700704 & 2.716465 & 0.000037 \\
\hline 17 & $\mathrm{H}$ & 1.239987 & 3.660646 & 0.000079 \\
\hline 18 & $\mathrm{H}$ & -1.239986 & 3.660646 & -0.000159 \\
\hline 19 & $\mathrm{H}$ & 1.208097 & -3.099694 & 0.000146 \\
\hline 20 & $\mathrm{H}$ & 4.651097 & 0.376845 & 0.000436 \\
\hline 21 & $\mathrm{H}$ & 3.380239 & 2.469999 & 0.000296 \\
\hline 22 & $\mathrm{H}$ & -1.208097 & -3.099694 & -0.000082 \\
\hline 23 & $\mathrm{H}$ & -4.651097 & 0.376845 & -0.000446 \\
\hline 24 & $\mathrm{H}$ & -3.380239 & 2.469999 & -0.000351 \\
\hline 25 & $\mathrm{C}$ & 3.687259 & -2.064118 & 0.000367 \\
\hline 26 & $\mathrm{H}$ & 4.770065 & -1.984401 & 0.000468 \\
\hline 27 & $\mathrm{C}$ & -3.687259 & -2.064117 & -0.000324 \\
\hline 28 & $\mathrm{H}$ & -3.282149 & -3.069521 & -0.000273 \\
\hline 29 & $\mathrm{H}$ & -4.770065 & -1.984400 & -0.000427 \\
\hline 30 & $\mathrm{H}$ & 3.282149 & -3.069521 & 0.000338 \\
\hline
\end{tabular}




\section{1,8-Pyrenoquinodimethane (2a)}

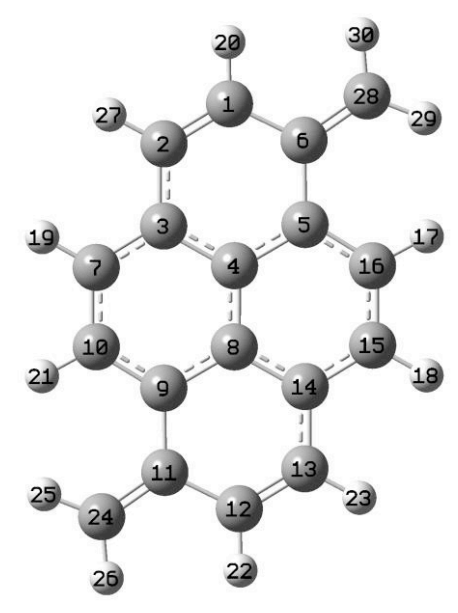

SCF Done: $\quad E(R B 3 L Y P)=-693.131182653 \quad$ A.U. after

\begin{tabular}{|c|c|c|c|c|}
\hline No. & Atom & $\mathrm{X}$ & $\mathrm{Y}$ & $\mathrm{Z}$ \\
\hline 1 & $\mathrm{C}$ & 3.363916 & -1.181886 & 0.000341 \\
\hline 2 & $\mathrm{C}$ & 2.340459 & -2.060927 & 0.000291 \\
\hline 3 & $\mathrm{C}$ & 0.962040 & -1.619637 & 0.000155 \\
\hline 4 & $\mathrm{C}$ & 0.680581 & -0.218334 & 0.000068 \\
\hline 5 & $\mathrm{C}$ & 1.751299 & 0.737822 & 0.000118 \\
\hline 6 & $\mathrm{C}$ & 3.156220 & 0.261848 & 0.000263 \\
\hline 7 & $\mathrm{C}$ & -0.094704 & -2.524152 & 0.000106 \\
\hline 8 & $\mathrm{C}$ & -0.680581 & 0.218334 & -0.000068 \\
\hline 9 & $\mathrm{C}$ & -1.751299 & -0.737822 & -0.000118 \\
\hline 10 & $\mathrm{C}$ & -1.423568 & -2.090965 & -0.000027 \\
\hline 11 & $\mathrm{C}$ & -3.156220 & -0.261848 & -0.000263 \\
\hline 12 & $\mathrm{C}$ & -3.363916 & 1.181886 & -0.000341 \\
\hline 13 & $\mathrm{C}$ & -2.340459 & 2.060927 & -0.000291 \\
\hline 14 & $\mathrm{C}$ & -0.962040 & 1.619637 & -0.000155 \\
\hline 15 & $\mathrm{C}$ & 0.094704 & 2.524152 & -0.000106 \\
\hline
\end{tabular}

7 cycles

\begin{tabular}{|c|c|c|c|c|}
\hline No. & Atom & $\mathrm{X}$ & $\mathrm{Y}$ & $\mathrm{Z}$ \\
\hline 16 & $\mathrm{C}$ & 1.423568 & 2.090965 & 0.000027 \\
\hline 17 & $\mathrm{H}$ & 2.209483 & 2.839129 & 0.000059 \\
\hline 18 & $\mathrm{H}$ & -0.119959 & 3.590172 & -0.000173 \\
\hline 19 & $\mathrm{H}$ & 0.119959 & -3.590172 & 0.000173 \\
\hline 20 & $\mathrm{H}$ & 4.391634 & -1.536127 & 0.000444 \\
\hline 21 & $\mathrm{H}$ & -2.209483 & -2.839129 & -0.000059 \\
\hline 22 & $\mathrm{H}$ & -4.391634 & 1.536127 & -0.000444 \\
\hline 23 & $\mathrm{H}$ & -2.532040 & 3.131435 & -0.000354 \\
\hline 24 & $\mathrm{C}$ & -4.239182 & -1.077696 & -0.000328 \\
\hline 25 & $\mathrm{H}$ & -4.173642 & -2.159672 & -0.000280 \\
\hline 26 & $\mathrm{H}$ & -5.241031 & -0.659300 & -0.000434 \\
\hline 27 & $\mathrm{H}$ & 2.532040 & -3.131435 & 0.000354 \\
\hline 28 & $\mathrm{C}$ & 4.239182 & 1.077696 & 0.000328 \\
\hline 29 & $\mathrm{H}$ & 4.173642 & 2.159672 & 0.000280 \\
\hline 30 & $\mathrm{H}$ & 5.241031 & 0.659300 & 0.000434 \\
\hline
\end{tabular}




\section{Syn-Diindenopyrene (3a)}

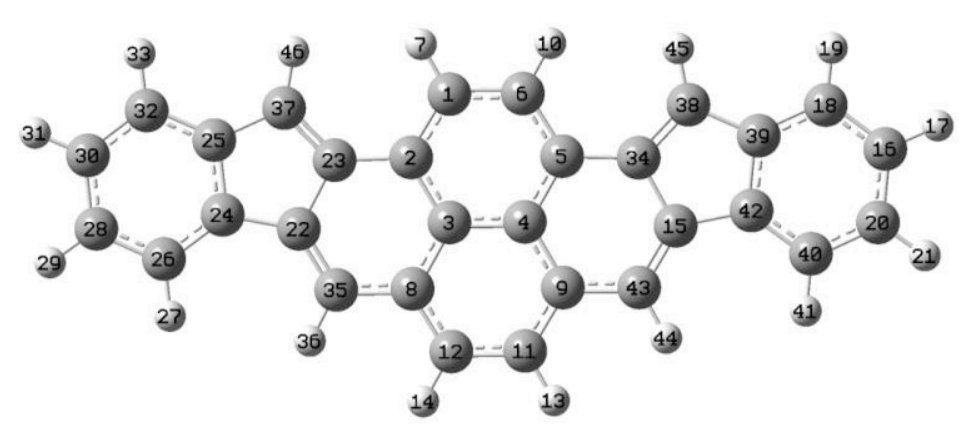

SCF Done: $\quad \mathrm{E}(\mathrm{RB} 3 \mathrm{LYP})=-1152.87229709$

A.U. after

\begin{tabular}{|c|c|c|c|c|}
\hline No. & Atom & $\mathrm{X}$ & $\mathrm{Y}$ & $\mathrm{Z}$ \\
\hline 1 & $\mathrm{C}$ & 0.000000 & 0.695548 & -1.993020 \\
\hline 2 & $\mathrm{C}$ & 0.000000 & 1.428081 & -0.800281 \\
\hline 3 & $\mathrm{C}$ & 0.000000 & 0.712638 & 0.438633 \\
\hline 4 & $\mathrm{C}$ & 0.000000 & -0.712638 & 0.438633 \\
\hline 5 & $\mathrm{C}$ & 0.000000 & -1.428081 & -0.800281 \\
\hline 6 & $\mathrm{C}$ & 0.000000 & -0.695548 & -1.993020 \\
\hline 7 & $\mathrm{H}$ & 0.000000 & 1.224252 & -2.942057 \\
\hline 8 & $\mathrm{C}$ & 0.000000 & 1.422120 & 1.690269 \\
\hline 9 & $\mathrm{C}$ & 0.000000 & -1.422120 & 1.690269 \\
\hline 10 & $\mathrm{H}$ & 0.000000 & -1.224252 & -2.942057 \\
\hline 11 & $\mathrm{C}$ & 0.000000 & -0.698114 & 2.883965 \\
\hline 12 & $\mathrm{C}$ & 0.000000 & 0.698114 & 2.883965 \\
\hline 13 & $\mathrm{H}$ & 0.000000 & -1.236537 & 3.828482 \\
\hline 14 & $\mathrm{H}$ & 0.000000 & 1.236537 & 3.828482 \\
\hline 15 & $\mathrm{C}$ & 0.000000 & -3.564331 & 0.535632 \\
\hline 16 & $\mathrm{C}$ & 0.000000 & -7.541678 & -0.887493 \\
\hline 17 & $\mathrm{H}$ & 0.000000 & -8.538490 & -1.320521 \\
\hline 18 & $\mathrm{C}$ & 0.000000 & -6.423613 & -1.729353 \\
\hline 19 & $\mathrm{H}$ & 0.000000 & -6.548491 & -2.809341 \\
\hline 20 & $\mathrm{C}$ & 0.000000 & -7.394158 & 0.503840 \\
\hline 21 & $\mathrm{H}$ & 0.000000 & -8.275595 & 1.139064 \\
\hline 22 & $\mathrm{C}$ & 0.000000 & 3.564331 & 0.535632 \\
\hline 23 & $\mathrm{C}$ & 0.000000 & 2.876610 & -0.759035 \\
\hline
\end{tabular}

7 cycles

\begin{tabular}{|c|c|c|c|c|}
\hline No. & Atom & $X$ & $\mathrm{Y}$ & Z \\
\hline 24 & $\mathrm{C}$ & 0.000000 & 5.003445 & 0.259308 \\
\hline 25 & $\mathrm{C}$ & 0.000000 & 5.150428 & -1.157348 \\
\hline 26 & $\mathrm{C}$ & 0.000000 & 6.119267 & 1.087052 \\
\hline 27 & $\mathrm{H}$ & 0.000000 & 6.014603 & 2.169511 \\
\hline 28 & $\mathrm{C}$ & 0.000000 & 7.394158 & 0.503840 \\
\hline 29 & $\mathrm{H}$ & 0.000000 & 8.275595 & 1.139064 \\
\hline 30 & $\mathrm{C}$ & 0.000000 & 7.541678 & -0.887493 \\
\hline 31 & $\mathrm{H}$ & 0.000000 & 8.538490 & -1.320521 \\
\hline 32 & $\mathrm{C}$ & 0.000000 & 6.423613 & -1.729353 \\
\hline 33 & $\mathrm{H}$ & 0.000000 & 6.548491 & -2.809341 \\
\hline 34 & $\mathrm{C}$ & 0.000000 & -2.876610 & -0.759035 \\
\hline 35 & $\mathrm{C}$ & 0.000000 & 2.865356 & 1.699448 \\
\hline 36 & $\mathrm{H}$ & 0.000000 & 3.371126 & 2.662891 \\
\hline 37 & $\mathrm{C}$ & 0.000000 & 3.824848 & -1.755605 \\
\hline 38 & $\mathrm{C}$ & 0.000000 & -3.824848 & -1.755605 \\
\hline 39 & $\mathrm{C}$ & 0.000000 & -5.150428 & -1.157348 \\
\hline 40 & $\mathrm{C}$ & 0.000000 & -6.119267 & 1.087052 \\
\hline 41 & $\mathrm{H}$ & 0.000000 & -6.014603 & 2.169511 \\
\hline 42 & $\mathrm{C}$ & 0.000000 & -5.003445 & 0.259308 \\
\hline 43 & $\mathrm{C}$ & 0.000000 & -2.865356 & 1.699448 \\
\hline 44 & $\mathrm{H}$ & 0.000000 & -3.371126 & 2.662891 \\
\hline 45 & $\mathrm{H}$ & 0.000000 & -3.639293 & -2.824103 \\
\hline 46 & $\mathrm{H}$ & 0.000000 & 3.639293 & -2.824103 \\
\hline
\end{tabular}




\section{Syn-Diindenopyrene Derivative 3b}
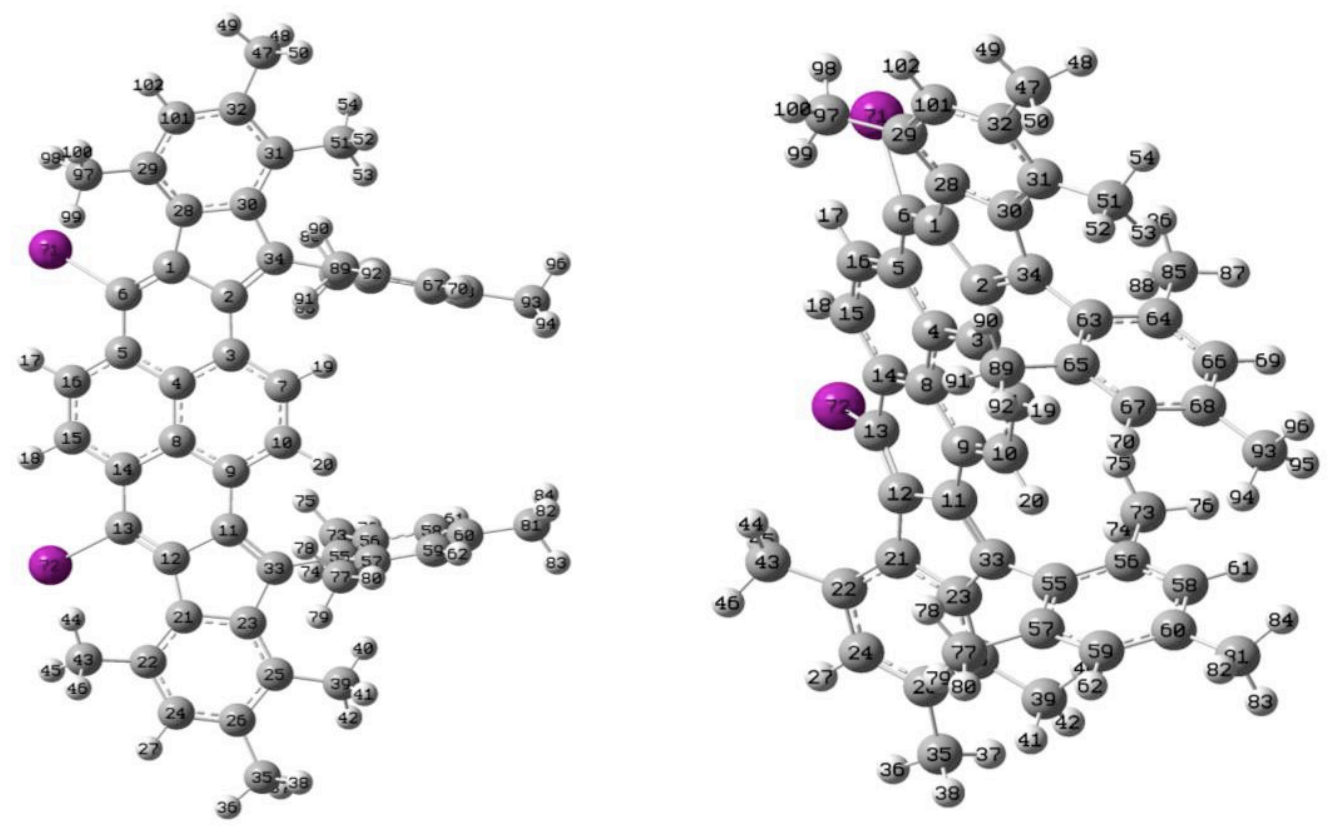

SCF Done: $\quad E($ RB3LYP $)=-2108.24412708$

A.U. after

\begin{tabular}{|c|c|c|c|c|}
\hline No. & Atom & $\mathrm{X}$ & $\mathrm{Y}$ & $\mathrm{Z}$ \\
\hline 1 & $\mathrm{C}$ & -1.134002 & -0.387163 & 3.594799 \\
\hline 2 & $\mathrm{C}$ & 0.157115 & -0.290905 & 2.885341 \\
\hline 3 & $\mathrm{C}$ & 0.196135 & -0.268447 & 1.433403 \\
\hline 4 & $\mathrm{C}$ & -1.038262 & -0.166442 & 0.717177 \\
\hline 5 & $\mathrm{C}$ & -2.278507 & -0.032403 & 1.436809 \\
\hline 6 & $\mathrm{C}$ & -2.262480 & -0.079290 & 2.892564 \\
\hline 7 & $\mathrm{C}$ & 1.379269 & -0.381272 & 0.695148 \\
\hline 8 & $\mathrm{C}$ & -1.038262 & -0.166442 & -0.717177 \\
\hline 9 & $\mathrm{C}$ & 0.196135 & -0.268447 & -1.433403 \\
\hline 10 & $\mathrm{C}$ & 1.379269 & -0.381272 & -0.695148 \\
\hline 11 & $\mathrm{C}$ & 0.157115 & -0.290905 & -2.885341 \\
\hline 12 & $\mathrm{C}$ & -1.134002 & -0.387163 & -3.594799 \\
\hline 13 & $\mathrm{C}$ & -2.262480 & -0.079290 & -2.892564 \\
\hline 14 & $\mathrm{C}$ & -2.278507 & -0.032403 & -1.436809 \\
\hline 15 & $\mathrm{C}$ & -3.451200 & 0.098243 & -0.696197 \\
\hline 16 & $\mathrm{C}$ & -3.451200 & 0.098243 & 0.696197 \\
\hline 17 & $\mathrm{H}$ & -4.400630 & 0.189654 & 1.207938 \\
\hline 18 & $\mathrm{H}$ & -4.400630 & 0.189654 & -1.207938 \\
\hline
\end{tabular}

\begin{tabular}{|c|c|c|c|c|}
\hline No. & Atom & $\mathrm{X}$ & $\mathrm{Y}$ & $\mathrm{Z}$ \\
\hline 52 & $\mathrm{H}$ & 3.270694 & -0.660638 & 6.857929 \\
\hline 53 & $\mathrm{H}$ & 3.026611 & 0.763776 & 5.850328 \\
\hline 54 & $\mathrm{H}$ & 2.675224 & 0.830880 & 7.577118 \\
\hline 55 & $\mathrm{C}$ & 2.603709 & 0.093313 & -3.537021 \\
\hline 56 & $\mathrm{C}$ & 3.075728 & 1.394269 & -3.256769 \\
\hline 57 & $\mathrm{C}$ & 3.503507 & -0.995386 & -3.543151 \\
\hline 58 & $\mathrm{C}$ & 4.437563 & 1.582455 & -2.999418 \\
\hline 59 & $\mathrm{C}$ & 4.856323 & -0.761393 & -3.275824 \\
\hline 60 & $\mathrm{C}$ & 5.346831 & 0.520213 & -3.007217 \\
\hline 61 & $\mathrm{H}$ & 4.796181 & 2.587582 & -2.785329 \\
\hline 62 & $\mathrm{H}$ & 5.544577 & -1.604727 & -3.277430 \\
\hline 63 & $\mathrm{C}$ & 2.603709 & 0.093313 & 3.537021 \\
\hline 64 & $\mathrm{C}$ & 3.075728 & 1.394269 & 3.256769 \\
\hline 65 & $\mathrm{C}$ & 3.503507 & -0.995386 & 3.543151 \\
\hline 66 & $\mathrm{C}$ & 4.437563 & 1.582455 & 2.999418 \\
\hline 67 & $\mathrm{C}$ & 4.856323 & -0.761393 & 3.275824 \\
\hline 68 & $\mathrm{C}$ & 5.346831 & 0.520213 & 3.007217 \\
\hline 69 & $\mathrm{H}$ & 4.796181 & 2.587582 & 2.785329 \\
\hline & & & & \\
\hline
\end{tabular}




\begin{tabular}{|c|c|c|c|c|c|c|c|c|c|}
\hline 19 & $\mathrm{H}$ & 2.324088 & -0.491970 & 1.209507 & 70 & $\mathrm{H}$ & 5.544577 & -1.604727 & 3.277430 \\
\hline 20 & $\mathrm{H}$ & 2.324088 & -0.491970 & -1.209507 & 71 & I & -4.035924 & 0.651254 & 3.900496 \\
\hline 21 & $\mathrm{C}$ & -0.818527 & -0.562229 & -5.020777 & 72 & I & -4.035924 & 0.651254 & -3.900496 \\
\hline 22 & $\mathrm{C}$ & -1.544617 & -1.087757 & -6.095002 & 73 & $\mathrm{C}$ & 2.130857 & 2.574484 & -3.226236 \\
\hline 23 & $\mathrm{C}$ & 0.575779 & -0.269241 & -5.172081 & 74 & $\mathrm{H}$ & 1.528759 & 2.635264 & -4.140175 \\
\hline 24 & $\mathrm{C}$ & -0.908695 & -1.081213 & -7.349768 & 75 & $\mathrm{H}$ & 1.427361 & 2.501226 & -2.388123 \\
\hline 25 & $\mathrm{C}$ & 1.179408 & -0.227255 & -6.440502 & 76 & $\mathrm{H}$ & 2.682832 & 3.513610 & -3.119197 \\
\hline 26 & $\mathrm{C}$ & 0.391047 & -0.623666 & -7.550945 & 77 & $\mathrm{C}$ & 3.018318 & -2.402523 & -3.812580 \\
\hline 27 & $\mathrm{H}$ & -1.458759 & -1.474025 & -8.202737 & 78 & $\mathrm{H}$ & 2.261484 & -2.711624 & -3.081393 \\
\hline 28 & $\mathrm{C}$ & -0.818527 & -0.562229 & 5.020777 & 79 & $\mathrm{H}$ & 2.552175 & -2.489008 & -4.800986 \\
\hline 29 & $\mathrm{C}$ & -1.544617 & -1.087757 & 6.095002 & 80 & $\mathrm{H}$ & 3.845889 & -3.117204 & -3.764715 \\
\hline 30 & $\mathrm{C}$ & 0.575779 & -0.269241 & 5.172081 & 81 & $\mathrm{C}$ & 6.818928 & 0.753850 & -2.757765 \\
\hline 31 & $\mathrm{C}$ & 1.179408 & -0.227255 & 6.440502 & 82 & $\mathrm{H}$ & 7.295377 & -0.127352 & -2.314700 \\
\hline 32 & $\mathrm{C}$ & 0.391047 & -0.623666 & 7.550945 & 83 & $\mathrm{H}$ & 7.351283 & 0.975205 & -3.692810 \\
\hline 33 & $\mathrm{C}$ & 1.160389 & -0.135355 & -3.825542 & 84 & $\mathrm{H}$ & 6.981975 & 1.602287 & -2.084292 \\
\hline 34 & $\mathrm{C}$ & 1.160389 & -0.135355 & 3.825542 & 85 & $\mathrm{C}$ & 2.130857 & 2.574484 & 3.226236 \\
\hline 35 & $\mathrm{C}$ & 0.963723 & -0.619451 & -8.950220 & 86 & $\mathrm{H}$ & 1.528759 & 2.635264 & 4.140175 \\
\hline 36 & $\mathrm{H}$ & 0.249708 & -1.040435 & -9.664436 & 87 & $\mathrm{H}$ & 2.682832 & 3.513610 & 3.119197 \\
\hline 37 & $\mathrm{H}$ & 1.207089 & 0.396955 & -9.287487 & 88 & $\mathrm{H}$ & 1.427361 & 2.501226 & 2.388123 \\
\hline 38 & $\mathrm{H}$ & 1.890171 & -1.203463 & -9.018334 & 89 & $\mathrm{C}$ & 3.018318 & -2.402523 & 3.812580 \\
\hline 39 & $\mathrm{C}$ & 2.610504 & 0.200802 & -6.683230 & 90 & $\mathrm{H}$ & 2.552175 & -2.489008 & 4.800986 \\
\hline 40 & $\mathrm{H}$ & 3.026611 & 0.763776 & -5.850328 & 91 & $\mathrm{H}$ & 2.261484 & -2.711624 & 3.081393 \\
\hline 41 & $\mathrm{H}$ & 3.270694 & -0.660638 & -6.857929 & 92 & $\mathrm{H}$ & 3.845889 & -3.117204 & 3.764715 \\
\hline 42 & $\mathrm{H}$ & 2.675224 & 0.830880 & -7.577118 & 93 & $\mathrm{C}$ & 6.818928 & 0.753850 & 2.757765 \\
\hline 43 & $\mathrm{C}$ & -2.880199 & -1.787139 & -5.993622 & 94 & $\mathrm{H}$ & 7.295377 & -0.127352 & 2.314700 \\
\hline 44 & $\mathrm{H}$ & -3.101439 & -2.104629 & -4.972413 & 95 & $\mathrm{H}$ & 6.981975 & 1.602287 & 2.084292 \\
\hline 45 & $\mathrm{H}$ & -3.712528 & -1.161045 & -6.332212 & 96 & $\mathrm{H}$ & 7.351283 & 0.975205 & 3.692810 \\
\hline 46 & $\mathrm{H}$ & -2.865982 & -2.681390 & -6.628022 & 97 & $\mathrm{C}$ & -2.880199 & -1.787139 & 5.993622 \\
\hline 47 & $\mathrm{C}$ & 0.963723 & -0.619451 & 8.950220 & 98 & $\mathrm{H}$ & -3.712528 & -1.161045 & 6.332212 \\
\hline 48 & $\mathrm{H}$ & 1.207089 & 0.396955 & 9.287487 & 99 & $\mathrm{H}$ & -3.101439 & -2.104629 & 4.972413 \\
\hline 49 & $\mathrm{H}$ & 0.249708 & -1.040435 & 9.664436 & 100 & $\mathrm{H}$ & -2.865980 & -2.681390 & 6.628022 \\
\hline 50 & $\mathrm{H}$ & 1.890171 & -1.203463 & 9.018334 & 101 & $\mathrm{C}$ & -0.908690 & -1.081210 & 7.349768 \\
\hline 51 & $\mathrm{C}$ & 2.610504 & 0.200802 & 6.683230 & 102 & $\mathrm{H}$ & -1.458750 & -1.474020 & 8.202737 \\
\hline
\end{tabular}


Anti-Diindenopyrene (4a)

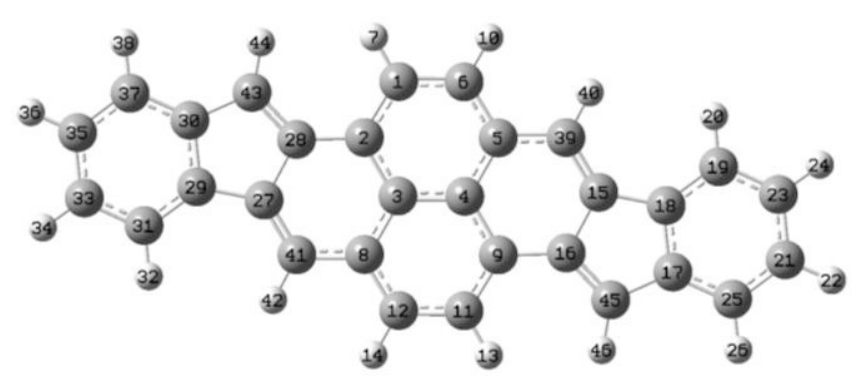

SCF Done: $\quad E(R B 3 L Y P)=-1152.87244244$

A.U. after

\begin{tabular}{|c|c|c|c|c|}
\hline No. & Atom & $\mathrm{X}$ & $\mathrm{Y}$ & $\mathrm{Z}$ \\
\hline 1 & $\mathrm{C}$ & -2.317916 & 1.035683 & 0.000000 \\
\hline 2 & $\mathrm{C}$ & -1.034376 & 1.589425 & 0.000000 \\
\hline 3 & $\mathrm{C}$ & 0.098077 & 0.705451 & 0.000000 \\
\hline 4 & $\mathrm{C}$ & -0.098077 & -0.705451 & 0.000000 \\
\hline 5 & $\mathrm{C}$ & -1.431217 & -1.233371 & 0.000000 \\
\hline 6 & $\mathrm{C}$ & -2.510507 & -0.344308 & 0.000000 \\
\hline 7 & $\mathrm{H}$ & -3.182606 & 1.693016 & 0.000000 \\
\hline 8 & $\mathrm{C}$ & 1.431217 & 1.233371 & 0.000000 \\
\hline 9 & $\mathrm{C}$ & 1.034376 & -1.589425 & 0.000000 \\
\hline 10 & $\mathrm{H}$ & -3.521827 & -0.743994 & 0.000000 \\
\hline 11 & $\mathrm{C}$ & 2.317916 & -1.035683 & 0.000000 \\
\hline 12 & $\mathrm{C}$ & 2.510507 & 0.344308 & 0.000000 \\
\hline 13 & $\mathrm{H}$ & 3.182606 & -1.693016 & 0.000000 \\
\hline 14 & $\mathrm{H}$ & 3.521827 & 0.743994 & 0.000000 \\
\hline 15 & $\mathrm{C}$ & -0.589547 & -3.516881 & 0.000000 \\
\hline 16 & $\mathrm{C}$ & 0.787661 & -3.016705 & 0.000000 \\
\hline 17 & $\mathrm{C}$ & 0.867077 & -5.322893 & 0.000000 \\
\hline 18 & $\mathrm{C}$ & -0.515809 & -4.979419 & 0.000000 \\
\hline 19 & $\mathrm{C}$ & -1.490607 & -5.970239 & 0.000000 \\
\hline 20 & $\mathrm{H}$ & -2.547992 & -5.716202 & 0.000000 \\
\hline 21 & $\mathrm{C}$ & 0.267832 & -7.653537 & 0.000000 \\
\hline 22 & $\mathrm{H}$ & 0.557424 & -8.701037 & 0.000000 \\
\hline 23 & $\mathrm{C}$ & -1.090194 & -7.312985 & 0.000000 \\
\hline
\end{tabular}

7 cycles

\begin{tabular}{|c|c|c|c|c|}
\hline No. & Atom & $\mathrm{X}$ & $\mathrm{Y}$ & $\mathrm{Z}$ \\
\hline 24 & $\mathrm{H}$ & -1.841717 & -8.097646 & 0.000000 \\
\hline 25 & $\mathrm{C}$ & 1.256395 & -6.664297 & 0.000000 \\
\hline 26 & $\mathrm{H}$ & 2.308590 & -6.938027 & 0.000000 \\
\hline 27 & $\mathrm{C}$ & 0.589547 & 3.516881 & 0.000000 \\
\hline 28 & $\mathrm{C}$ & -0.787661 & 3.016705 & 0.000000 \\
\hline 29 & $\mathrm{C}$ & 0.515809 & 4.979419 & 0.000000 \\
\hline 30 & $\mathrm{C}$ & -0.867077 & 5.322893 & 0.000000 \\
\hline 31 & $\mathrm{C}$ & 1.490607 & 5.970239 & 0.000000 \\
\hline 32 & $\mathrm{H}$ & 2.547992 & 5.716202 & 0.000000 \\
\hline 33 & $\mathrm{C}$ & 1.090194 & 7.312985 & 0.000000 \\
\hline 34 & $\mathrm{H}$ & 1.841717 & 8.097646 & 0.000000 \\
\hline 35 & $\mathrm{C}$ & -0.267832 & 7.653537 & 0.000000 \\
\hline 36 & $\mathrm{H}$ & -0.557424 & 8.701037 & 0.000000 \\
\hline 37 & $\mathrm{C}$ & -1.256395 & 6.664297 & 0.000000 \\
\hline 38 & $\mathrm{H}$ & -2.308590 & 6.938027 & 0.000000 \\
\hline 39 & $\mathrm{C}$ & -1.643397 & -2.660751 & 0.000000 \\
\hline 40 & $\mathrm{H}$ & -2.668544 & -3.025822 & 0.000000 \\
\hline 41 & $\mathrm{C}$ & 1.643397 & 2.660751 & 0.000000 \\
\hline 42 & $\mathrm{H}$ & 2.668544 & 3.025822 & 0.000000 \\
\hline 43 & $\mathrm{C}$ & -1.643397 & 4.095669 & 0.000000 \\
\hline 44 & $\mathrm{H}$ & -2.727345 & 4.060745 & 0.000000 \\
\hline 45 & $\mathrm{C}$ & 1.643397 & -4.095669 & 0.000000 \\
\hline 46 & $\mathrm{H}$ & 2.727345 & -4.060745 & 0.000000 \\
\hline
\end{tabular}




\section{Anti-Diindenopyrene Derivative 4b}
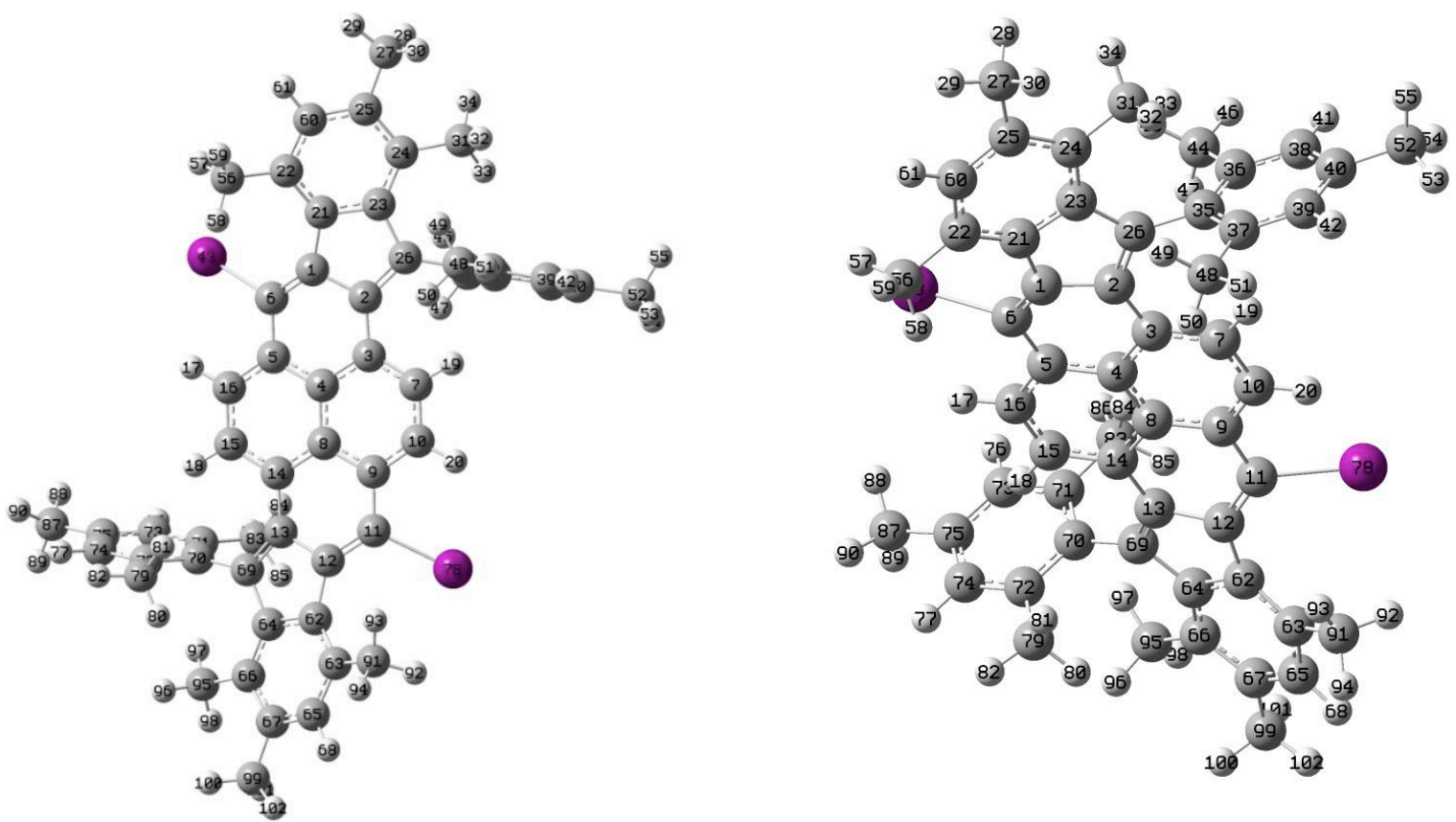

SCF Done: $\quad \mathrm{E}(\mathrm{RB} 3 \mathrm{LYP})=-2108.24484387 \quad$ A.U. after $\quad 7$ cycles

\begin{tabular}{|c|c|c|c|c|c|c|c|c|c|}
\hline No. & Atom & $X$ & Y & Z & No. & Atom & $X$ & Y & Z \\
\hline 1 & $\mathrm{C}$ & 1.345750 & 3.333686 & -0.503256 & 52 & $\mathrm{C}$ & -6.238113 & 5.302206 & 1.454685 \\
\hline 2 & $\mathrm{C}$ & -0.098071 & 3.125477 & -0.280973 & 53 & $\mathrm{H}$ & -6.921938 & 5.119403 & 0.618898 \\
\hline 3 & $\mathrm{C}$ & -0.649992 & 1.783612 & -0.252341 & 54 & $\mathrm{H}$ & -6.551438 & 4.671071 & 2.293493 \\
\hline 4 & $\mathrm{C}$ & 0.253307 & 0.670462 & -0.268126 & 55 & $\mathrm{H}$ & -6.376601 & 6.346529 & 1.765918 \\
\hline 5 & $\mathrm{C}$ & 1.673178 & 0.898132 & -0.253161 & 56 & $\mathrm{C}$ & 3.698970 & 5.002742 & -2.063014 \\
\hline 6 & $\mathrm{C}$ & 2.171129 & 2.266031 & -0.301687 & 57 & $\mathrm{H}$ & 4.647635 & 5.005968 & -1.515727 \\
\hline 7 & $\mathrm{C}$ & -2.021595 & 1.517401 & -0.245132 & 58 & $\mathrm{H}$ & 3.514651 & 3.979067 & -2.395864 \\
\hline 8 & $\mathrm{C}$ & -0.253307 & -0.670462 & -0.268126 & 59 & $\mathrm{H}$ & 3.834118 & 5.627430 & -2.953919 \\
\hline 9 & $\mathrm{C}$ & -1.673178 & -0.898132 & -0.253161 & 60 & $\mathrm{C}$ & 2.402080 & 6.945186 & -1.185066 \\
\hline 10 & $\mathrm{C}$ & -2.513398 & 0.216203 & -0.239892 & 61 & $\mathrm{H}$ & 3.180038 & 7.560717 & -1.632803 \\
\hline 11 & $\mathrm{C}$ & -2.171129 & -2.266031 & -0.301687 & 62 & $\mathrm{C}$ & -1.542117 & -4.783310 & -0.649772 \\
\hline 12 & $\mathrm{C}$ & -1.345750 & -3.333686 & -0.503256 & 63 & $\mathrm{C}$ & -2.551542 & -5.548560 & -1.245100 \\
\hline 13 & $\mathrm{C}$ & 0.098071 & -3.125477 & -0.280973 & 64 & $\mathrm{C}$ & -0.325074 & -5.409218 & -0.225618 \\
\hline 14 & $\mathrm{C}$ & 0.649992 & -1.783612 & -0.252341 & 65 & $\mathrm{C}$ & -2.402080 & -6.945186 & -1.185066 \\
\hline 15 & $\mathrm{C}$ & 2.021595 & -1.517401 & -0.245132 & 66 & $\mathrm{C}$ & -0.214538 & -6.808242 & -0.132492 \\
\hline 16 & $\mathrm{C}$ & 2.513398 & -0.216203 & -0.239892 & 67 & $\mathrm{C}$ & -1.303701 & -7.579962 & -0.607953 \\
\hline 17 & $\mathrm{H}$ & 3.586766 & -0.074709 & -0.245795 & 68 & $\mathrm{H}$ & -3.180038 & -7.560717 & -1.632803 \\
\hline
\end{tabular}




\begin{tabular}{|c|c|c|c|c|}
\hline 18 & $\mathrm{H}$ & 2.729429 & -2.334637 & -0.258651 \\
\hline 19 & $\mathrm{H}$ & -2.729429 & 2.334637 & -0.258651 \\
\hline 20 & $\mathrm{H}$ & -3.586766 & 0.074709 & -0.245795 \\
\hline 21 & $\mathrm{C}$ & 1.542117 & 4.783310 & -0.649772 \\
\hline 22 & $\mathrm{C}$ & 2.551542 & 5.548560 & -1.245100 \\
\hline 23 & $\mathrm{C}$ & 0.325074 & 5.409218 & -0.225618 \\
\hline 24 & $\mathrm{C}$ & 0.214538 & 6.808242 & -0.132492 \\
\hline 25 & $\mathrm{C}$ & 1.303701 & 7.579962 & -0.607953 \\
\hline 26 & $\mathrm{C}$ & -0.683580 & 4.355215 & -0.029912 \\
\hline 27 & $\mathrm{C}$ & 1.268618 & 9.091139 & -0.559548 \\
\hline 28 & $\mathrm{H}$ & 1.261990 & 9.465761 & 0.472750 \\
\hline 29 & $\mathrm{H}$ & 2.145679 & 9.516210 & -1.056779 \\
\hline 30 & $\mathrm{H}$ & 0.375563 & 9.499305 & -1.048928 \\
\hline 31 & $\mathrm{C}$ & -0.997285 & 7.520741 & 0.428310 \\
\hline 32 & $\mathrm{H}$ & -1.668180 & 7.876306 & -0.366586 \\
\hline 33 & $\mathrm{H}$ & -1.587312 & 6.884884 & 1.086042 \\
\hline 34 & $\mathrm{H}$ & -0.693266 & 8.402671 & 1.001460 \\
\hline 35 & $\mathrm{C}$ & -2.105221 & 4.586601 & 0.349343 \\
\hline 36 & $\mathrm{C}$ & -2.513398 & 4.443354 & 1.694008 \\
\hline 37 & $\mathrm{C}$ & -3.049461 & 4.944206 & -0.637794 \\
\hline 38 & $\mathrm{C}$ & -3.852909 & 4.670746 & 2.025252 \\
\hline 39 & $\mathrm{C}$ & -4.379899 & 5.158096 & -0.261534 \\
\hline 40 & $\mathrm{C}$ & -4.803126 & 5.032451 & 1.064979 \\
\hline 41 & $\mathrm{H}$ & -4.161886 & 4.560214 & 3.063066 \\
\hline 42 & $\mathrm{H}$ & -5.104376 & 5.428479 & -1.027533 \\
\hline 43 & I & 4.242487 & 2.557707 & 0.263027 \\
\hline 44 & $\mathrm{C}$ & -1.527784 & 4.044586 & 2.769433 \\
\hline 45 & $\mathrm{H}$ & -0.642519 & 4.691073 & 2.768258 \\
\hline 46 & $\mathrm{H}$ & -1.988586 & 4.101017 & 3.760630 \\
\hline 47 & $\mathrm{H}$ & -1.170488 & 3.018039 & 2.624182 \\
\hline 48 & $\mathrm{C}$ & -2.644635 & 5.074611 & -2.089611 \\
\hline 49 & $\mathrm{H}$ & -1.870018 & 5.837681 & -2.229196 \\
\hline 50 & $\mathrm{H}$ & -2.231171 & 4.134981 & -2.475956 \\
\hline 51 & $\mathrm{H}$ & -3.503283 & 5.346308 & -2.711555 \\
\hline
\end{tabular}

\begin{tabular}{|l|l|l|l|l|}
\hline 69 & $\mathrm{C}$ & 0.683580 & -4.355215 & -0.029912 \\
\hline 70 & $\mathrm{C}$ & 2.105221 & -4.586601 & 0.349343 \\
\hline 71 & $\mathrm{C}$ & 2.513398 & -4.443354 & 1.694008 \\
\hline 72 & $\mathrm{C}$ & 3.049461 & -4.944206 & -0.637794 \\
\hline 73 & $\mathrm{C}$ & 3.852909 & -4.670746 & 2.025252 \\
\hline 74 & $\mathrm{C}$ & 4.379899 & -5.158096 & -0.261534 \\
\hline 75 & $\mathrm{C}$ & 4.803126 & -5.032451 & 1.064979 \\
\hline 76 & $\mathrm{H}$ & 4.161886 & -4.560214 & 3.063066 \\
\hline 77 & $\mathrm{H}$ & 5.104376 & -5.428479 & -1.027533 \\
\hline 78 & $\mathrm{I}$ & -4.242487 & -2.557707 & 0.263027 \\
\hline 79 & $\mathrm{C}$ & 2.644635 & -5.074611 & -2.089611 \\
\hline 80 & $\mathrm{H}$ & 1.870018 & -5.837681 & -2.229196 \\
\hline 81 & $\mathrm{H}$ & 2.231171 & -4.134981 & -2.475956 \\
\hline 82 & $\mathrm{H}$ & 3.503283 & -5.346308 & -2.711555 \\
\hline 83 & $\mathrm{C}$ & 1.527784 & -4.044586 & 2.769433 \\
\hline 84 & $\mathrm{H}$ & 1.170488 & -3.018039 & 2.624182 \\
\hline 85 & $\mathrm{H}$ & 0.642519 & -4.691073 & 2.768258 \\
\hline 86 & $\mathrm{H}$ & 1.988586 & -4.101017 & 3.760630 \\
\hline 87 & $\mathrm{C}$ & 6.238113 & -5.302206 & 1.454685 \\
\hline 88 & $\mathrm{H}$ & 6.551438 & -4.671071 & 2.293493 \\
\hline 89 & $\mathrm{H}$ & 6.376601 & -6.346529 & 1.765918 \\
\hline 90 & $\mathrm{H}$ & 6.921938 & -5.119403 & 0.618898 \\
\hline 91 & $\mathrm{C}$ & -3.698970 & -5.002742 & -2.063014 \\
\hline 92 & $\mathrm{H}$ & -4.647635 & -5.005968 & -1.515727 \\
\hline 93 & $\mathrm{H}$ & -3.514651 & -3.979067 & -2.395864 \\
\hline 94 & $\mathrm{H}$ & -3.834118 & -5.627430 & -2.953919 \\
\hline 95 & $\mathrm{C}$ & 0.997285 & -7.520741 & 0.428310 \\
\hline 96 & $\mathrm{H}$ & 1.668180 & -7.876306 & -0.366586 \\
\hline 97 & $\mathrm{H}$ & 1.587312 & -6.884884 & 1.086042 \\
\hline 98 & $\mathrm{H}$ & 0.693266 & -8.402671 & 1.001460 \\
\hline 99 & $\mathrm{C}$ & -1.268618 & -9.091139 & -0.559548 \\
\hline 100 & $\mathrm{H}$ & -0.375563 & -9.499305 & -1.048928 \\
\hline $\mathrm{H}$ & -1.261990 & -9.465761 & 0.472750 \\
\hline $\mathrm{H}$ & -2.145679 & -9.516210 & -1.056779 \\
\hline
\end{tabular}




\section{Cvcloheptapentalenoindenophenalene 8a}

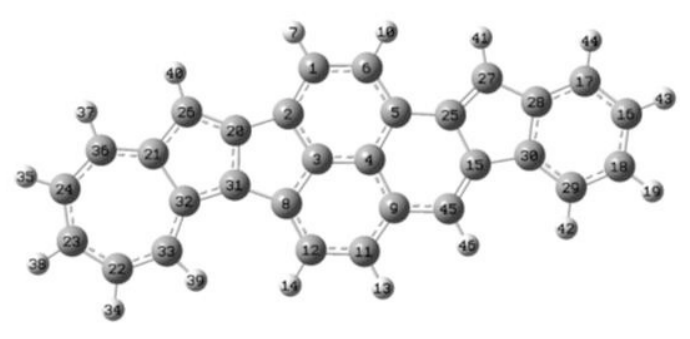

SCF Done: $\quad E($ RB3LYP $)=-1152.82541944$

A.U. after

\begin{tabular}{|c|c|c|c|c|}
\hline No. & Atom & $\mathrm{X}$ & Y & Z \\
\hline 1 & $\mathrm{C}$ & -2.374962 & 1.049603 & 0.000000 \\
\hline 2 & $\mathrm{C}$ & -1.085212 & 1.566579 & 0.000000 \\
\hline 3 & $\mathrm{C}$ & 0.000000 & 0.649711 & 0.000000 \\
\hline 4 & $\mathrm{C}$ & -0.161625 & -0.732402 & 0.000000 \\
\hline 5 & $\mathrm{C}$ & -1.492720 & -1.257633 & 0.000000 \\
\hline 6 & $\mathrm{C}$ & -2.561715 & -0.348916 & 0.000000 \\
\hline 7 & $\mathrm{H}$ & -3.245111 & 1.700861 & 0.000000 \\
\hline 8 & $\mathrm{C}$ & 1.273322 & 1.306875 & 0.000000 \\
\hline 9 & $\mathrm{C}$ & 1.019849 & -1.544808 & 0.000000 \\
\hline 10 & $\mathrm{H}$ & -3.577960 & -0.733858 & 0.000000 \\
\hline 11 & $\mathrm{C}$ & 2.264057 & -0.903261 & 0.000000 \\
\hline 12 & $\mathrm{C}$ & 2.406995 & 0.504255 & 0.000000 \\
\hline 13 & $\mathrm{H}$ & 3.163276 & -1.515441 & 0.000000 \\
\hline 14 & $\mathrm{H}$ & 3.406721 & 0.929649 & 0.000000 \\
\hline 15 & $\mathrm{C}$ & -0.389374 & -3.527123 & 0.000000 \\
\hline 16 & $\mathrm{C}$ & -2.239106 & -7.328517 & 0.000000 \\
\hline 17 & $\mathrm{C}$ & -2.954519 & -6.126592 & 0.000000 \\
\hline 18 & $\mathrm{C}$ & -0.839234 & -7.330503 & 0.000000 \\
\hline 19 & $\mathrm{H}$ & -0.302524 & -8.275307 & 0.000000 \\
\hline 20 & $\mathrm{C}$ & -0.467613 & 2.891379 & 0.000000 \\
\hline 21 & $\mathrm{C}$ & 0.423137 & 4.981638 & 0.000000 \\
\hline 22 & $\mathrm{C}$ & 3.560140 & 5.531629 & 0.000000 \\
\hline 23 & $\mathrm{C}$ & 2.989730 & 6.815356 & 0.000000 \\
\hline
\end{tabular}

16 cycles

\begin{tabular}{|c|c|c|c|c|}
\hline No. & Atom & $\mathrm{X}$ & $\mathrm{Y}$ & $\mathrm{Z}$ \\
\hline 24 & $\mathrm{C}$ & 1.648600 & 7.186890 & 0.000000 \\
\hline 25 & $\mathrm{C}$ & -1.611132 & -2.703967 & 0.000000 \\
\hline 26 & $\mathrm{C}$ & -0.790301 & 4.244485 & 0.000000 \\
\hline 27 & $\mathrm{C}$ & -2.700959 & -3.540879 & 0.000000 \\
\hline 28 & $\mathrm{C}$ & -2.248207 & -4.922230 & 0.000000 \\
\hline 29 & $\mathrm{C}$ & -0.122079 & -6.126919 & 0.000000 \\
\hline 30 & $\mathrm{C}$ & -0.823696 & -4.926558 & 0.000000 \\
\hline 31 & $\mathrm{C}$ & 0.963999 & 2.729772 & 0.000000 \\
\hline 32 & $\mathrm{C}$ & 1.559492 & 3.995856 & 0.000000 \\
\hline 33 & $\mathrm{C}$ & 2.927323 & 4.292252 & 0.000000 \\
\hline 34 & $\mathrm{H}$ & 4.647581 & 5.502793 & 0.000000 \\
\hline 35 & $\mathrm{H}$ & 1.453909 & 8.257154 & 0.000000 \\
\hline 36 & $\mathrm{C}$ & 0.508479 & 6.366943 & 0.000000 \\
\hline 37 & $\mathrm{H}$ & -0.446844 & 6.891062 & 0.000000 \\
\hline 38 & $\mathrm{H}$ & 3.702337 & 7.638404 & 0.000000 \\
\hline 39 & $\mathrm{H}$ & 3.589813 & 3.428071 & 0.000000 \\
\hline 40 & $\mathrm{H}$ & -1.778671 & 4.688520 & 0.000000 \\
\hline 41 & $\mathrm{H}$ & -3.743109 & -3.240140 & 0.000000 \\
\hline 42 & $\mathrm{H}$ & 0.965370 & -6.140113 & 0.000000 \\
\hline 43 & $\mathrm{H}$ & -2.776598 & -8.273123 & 0.000000 \\
\hline 44 & $\mathrm{H}$ & -4.041763 & -6.133320 & 0.000000 \\
\hline 45 & $\mathrm{C}$ & 0.856943 & -2.979250 & 0.000000 \\
\hline 46 & $\mathrm{H}$ & 1.745895 & -3.607213 & 0.000000 \\
\hline
\end{tabular}




\section{Cvcloheptapentalenoindenophenalene Derivative 8b}
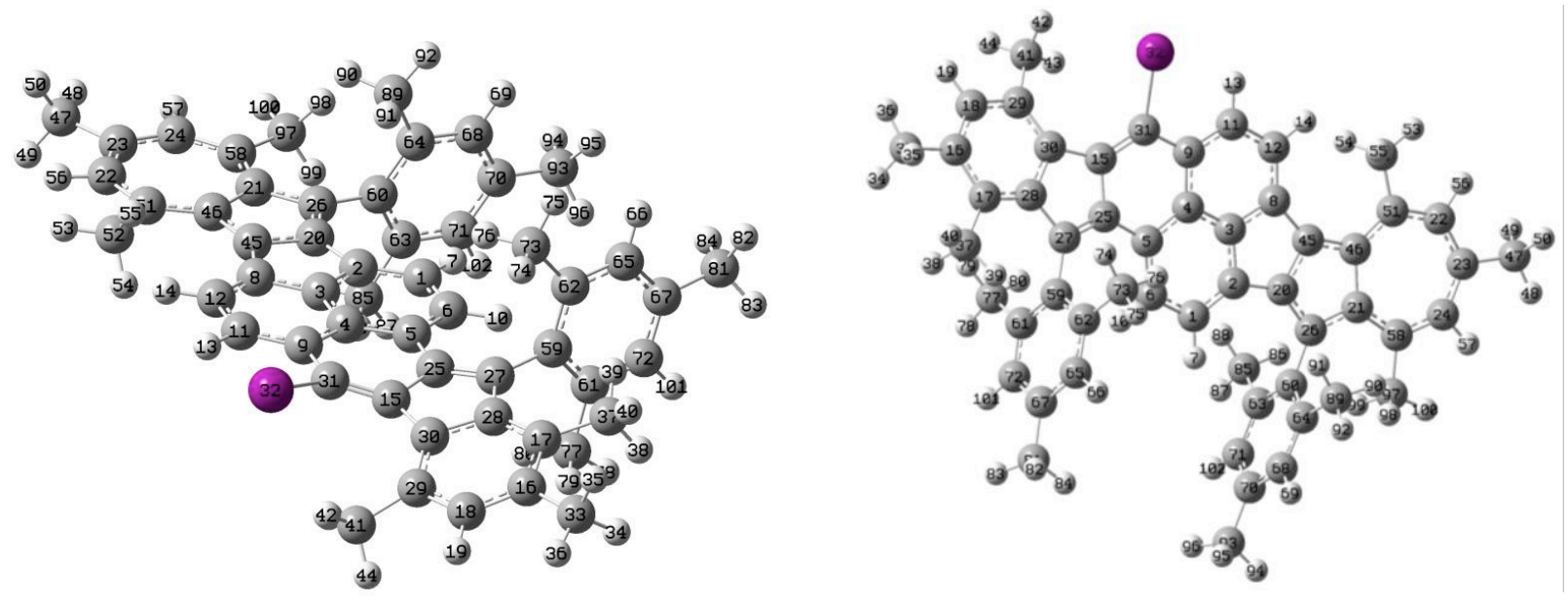

SCF Done: $\quad \mathrm{E}(\mathrm{RB} 3 \mathrm{LYP})=-2097.43470388 \quad$ A.U. after 4 cycles

\begin{tabular}{|c|c|c|c|c|c|c|c|c|c|}
\hline No. & Atom & $X$ & Y & Z & No. & Atom & $X$ & Y & Z \\
\hline 1 & $\mathrm{C}$ & 1.084828 & 1.393336 & 0.220609 & 52 & $\mathrm{C}$ & 4.202726 & -4.468971 & -0.120936 \\
\hline 2 & $\mathrm{C}$ & 1.762972 & 0.184301 & 0.149117 & 53 & $\mathrm{H}$ & 4.732822 & -5.424170 & -0.152348 \\
\hline 3 & $\mathrm{C}$ & 1.010965 & -1.016199 & 0.094529 & 54 & $\mathrm{H}$ & 3.557541 & -4.469044 & 0.763206 \\
\hline 4 & $\mathrm{C}$ & -0.387588 & -1.032886 & 0.107612 & 55 & $\mathrm{H}$ & 3.558436 & -4.412014 & -1.004387 \\
\hline 5 & $\mathrm{C}$ & -1.084013 & 0.214450 & 0.170670 & 56 & $\mathrm{H}$ & 6.688488 & -4.736756 & -0.179382 \\
\hline 6 & $\mathrm{C}$ & -0.323484 & 1.394669 & 0.230411 & 57 & $\mathrm{H}$ & 8.784803 & -1.123048 & -0.046301 \\
\hline 7 & $\mathrm{H}$ & 1.618892 & 2.337057 & 0.271532 & 58 & $\mathrm{C}$ & 6.777324 & -0.506845 & 0.027847 \\
\hline 8 & $\mathrm{C}$ & 1.853263 & -2.177476 & 0.012226 & 59 & $\mathrm{C}$ & -3.223578 & 2.554389 & -0.268951 \\
\hline 9 & $\mathrm{C}$ & -1.048399 & -2.303383 & 0.026190 & 60 & $\mathrm{C}$ & 4.553587 & 1.849331 & 0.203816 \\
\hline 10 & $\mathrm{H}$ & -0.831345 & 2.347830 & 0.298981 & 61 & $\mathrm{C}$ & -3.231702 & 3.486154 & 0.791402 \\
\hline 11 & $\mathrm{C}$ & -0.234307 & -3.434483 & -0.062297 & 62 & $\mathrm{C}$ & -2.955083 & 2.989287 & -1.585712 \\
\hline 12 & $\mathrm{C}$ & 1.177329 & -3.391637 & -0.064949 & 63 & $\mathrm{C}$ & 4.602203 & 2.487623 & 1.462145 \\
\hline 13 & $\mathrm{H}$ & -0.701069 & -4.410791 & -0.121245 & 64 & $\mathrm{C}$ & 4.538673 & 2.628744 & -0.973591 \\
\hline 14 & $\mathrm{H}$ & 1.689463 & -4.340339 & -0.131736 & 65 & $\mathrm{C}$ & -2.709830 & 4.346275 & -1.816713 \\
\hline 15 & $\mathrm{C}$ & -3.217023 & -1.169469 & 0.336037 & 66 & $\mathrm{H}$ & -2.512230 & 4.677184 & -2.834770 \\
\hline 16 & $\mathrm{C}$ & -7.206539 & 0.292209 & 0.508972 & 67 & $\mathrm{C}$ & -2.712037 & 5.286353 & -0.780853 \\
\hline 17 & $\mathrm{C}$ & -6.109856 & 1.086749 & 0.089382 & 68 & $\mathrm{C}$ & 4.609538 & 4.022344 & -0.872487 \\
\hline 18 & $\mathrm{C}$ & -6.979989 & -0.985604 & 1.014016 & 69 & $\mathrm{H}$ & 4.601055 & 4.615921 & -1.784996 \\
\hline 19 & $\mathrm{H}$ & -7.822882 & -1.537576 & 1.425795 & 70 & $\mathrm{C}$ & 4.684399 & 4.671601 & 0.363595 \\
\hline 20 & $\mathrm{C}$ & 3.154619 & -0.234011 & 0.107477 & 71 & $\mathrm{C}$ & 4.672375 & 3.884129 & 1.518504 \\
\hline 21 & $\mathrm{C}$ & 5.380075 & -0.690117 & 0.046244 & 72 & $\mathrm{C}$ & -2.976250 & 4.833831 & 0.515031 \\
\hline
\end{tabular}




\begin{tabular}{|c|c|c|c|c|}
\hline 22 & $\mathrm{C}$ & 6.512535 & -3.664065 & -0.125118 \\
\hline 23 & $\mathrm{C}$ & 7.685191 & -2.894069 & -0.111348 \\
\hline 24 & $\mathrm{C}$ & 7.768017 & -1.507483 & -0.044260 \\
\hline 25 & $\mathrm{C}$ & -2.536374 & 0.142266 & 0.196205 \\
\hline 26 & $\mathrm{C}$ & 4.409541 & 0.365536 & 0.121632 \\
\hline 27 & $\mathrm{C}$ & -3.496589 & 1.115717 & 0.004004 \\
\hline 28 & $\mathrm{C}$ & -4.830984 & 0.508254 & 0.152626 \\
\hline 29 & $\mathrm{C}$ & -5.712967 & -1.595693 & 1.048528 \\
\hline 30 & $\mathrm{C}$ & -4.650461 & -0.871561 & 0.495690 \\
\hline 31 & $\mathrm{C}$ & -2.502369 & -2.307180 & 0.080208 \\
\hline 32 & I & -3.466667 & -4.138041 & -0.558605 \\
\hline 33 & $\mathrm{C}$ & -8.616559 & 0.837311 & 0.481305 \\
\hline 34 & $\mathrm{H}$ & -8.705259 & 1.780093 & 1.035811 \\
\hline 35 & $\mathrm{H}$ & -8.954420 & 1.042220 & -0.543402 \\
\hline 36 & $\mathrm{H}$ & -9.318711 & 0.123086 & 0.922006 \\
\hline 37 & $\mathrm{C}$ & -6.375170 & 2.500258 & -0.381801 \\
\hline 38 & $\mathrm{H}$ & -6.567063 & 3.181428 & 0.459482 \\
\hline 39 & $\mathrm{H}$ & -5.545895 & 2.915176 & -0.951191 \\
\hline 40 & $\mathrm{H}$ & -7.265845 & 2.531430 & -1.019104 \\
\hline 41 & $\mathrm{C}$ & -5.588308 & -2.897270 & 1.806399 \\
\hline 42 & $\mathrm{H}$ & -5.908255 & -3.763652 & 1.217998 \\
\hline 43 & $\mathrm{H}$ & -4.563815 & -3.083368 & 2.135720 \\
\hline 44 & $\mathrm{H}$ & -6.225577 & -2.855301 & 2.697844 \\
\hline 45 & $\mathrm{C}$ & 3.240877 & -1.662442 & 0.025671 \\
\hline 46 & $\mathrm{C}$ & 4.615456 & -2.001005 & -0.012266 \\
\hline 47 & $\mathrm{C}$ & 8.986253 & -3.673829 & -0.174714 \\
\hline 48 & $\mathrm{H}$ & 9.858210 & -3.014141 & -0.170036 \\
\hline 49 & $\mathrm{H}$ & 9.076094 & -4.358161 & 0.678487 \\
\hline 50 & $\mathrm{H}$ & 9.032469 & -4.288632 & -1.082444 \\
\hline 51 & $\mathrm{C}$ & 5.166083 & -3.303635 & -0.083509 \\
\hline
\end{tabular}

\begin{tabular}{|c|c|c|c|c|}
\hline 73 & $\mathrm{C}$ & -2.930262 & 2.009394 & -2.737111 \\
\hline 74 & $\mathrm{H}$ & -3.839290 & 1.397723 & -2.769174 \\
\hline 75 & $\mathrm{H}$ & -2.838008 & 2.533326 & -3.693749 \\
\hline 76 & $\mathrm{H}$ & -2.085159 & 1.315768 & -2.651042 \\
\hline 77 & $\mathrm{C}$ & -3.499119 & 3.042671 & 2.212524 \\
\hline 78 & $\mathrm{H}$ & -3.459543 & 3.892270 & 2.901457 \\
\hline 79 & $\mathrm{H}$ & -4.483314 & 2.570276 & 2.312226 \\
\hline 80 & $\mathrm{H}$ & -2.761362 & 2.302322 & 2.544740 \\
\hline 81 & $\mathrm{C}$ & -2.413537 & 6.742597 & -1.053885 \\
\hline 82 & $\mathrm{H}$ & -2.837070 & 7.066303 & -2.011297 \\
\hline 83 & $\mathrm{H}$ & -2.818936 & 7.389096 & -0.268396 \\
\hline 84 & $\mathrm{H}$ & -1.331494 & 6.925622 & -1.101461 \\
\hline 85 & $\mathrm{C}$ & 4.568119 & 1.683510 & 2.743213 \\
\hline 86 & $\mathrm{H}$ & 5.366499 & 0.932644 & 2.775614 \\
\hline 87 & $\mathrm{H}$ & 4.678144 & 2.334104 & 3.616615 \\
\hline 88 & $\mathrm{H}$ & 3.623125 & 1.136670 & 2.845352 \\
\hline 89 & $\mathrm{C}$ & 4.436466 & 1.978017 & -2.335569 \\
\hline 90 & $\mathrm{H}$ & 5.241213 & 1.252323 & -2.503785 \\
\hline 91 & $\mathrm{H}$ & 3.494066 & 1.428461 & -2.445124 \\
\hline 92 & $\mathrm{H}$ & 4.485002 & 2.727676 & -3.131758 \\
\hline 93 & $\mathrm{C}$ & 4.794941 & 6.176731 & 0.446967 \\
\hline 94 & $\mathrm{H}$ & 5.842856 & 6.503382 & 0.401455 \\
\hline 95 & $\mathrm{H}$ & 4.268825 & 6.664318 & -0.381154 \\
\hline 96 & $\mathrm{H}$ & 4.377384 & 6.558206 & 1.385048 \\
\hline 97 & $\mathrm{C}$ & 7.352498 & 0.901368 & 0.087961 \\
\hline 98 & $\mathrm{H}$ & 7.015935 & 1.517058 & -0.750331 \\
\hline 99 & $\mathrm{H}$ & 7.055099 & 1.427277 & 0.999035 \\
\hline 100 & $\mathrm{H}$ & 8.444693 & 0.869071 & 0.061792 \\
\hline 101 & $\mathrm{H}$ & -2.986351 & 5.548529 & 1.335954 \\
\hline 102 & $\mathrm{H}$ & 4.714284 & 4.368738 & 2.492355 \\
\hline
\end{tabular}




\section{Cvcloheptapentalenoindenophenalene 9a}

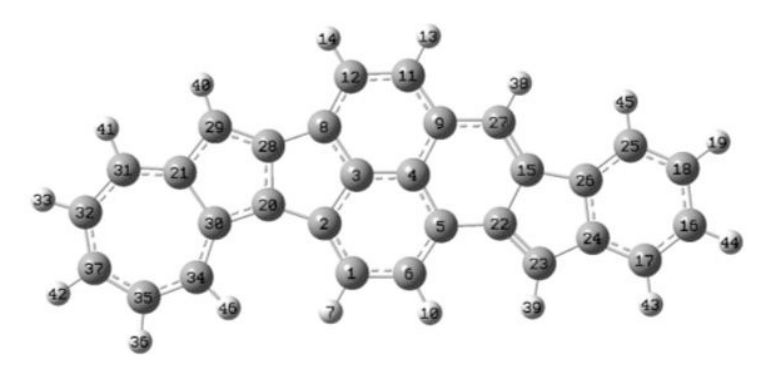

SCF Done: $\quad E($ RB3LYP $)=-1152.82452921$

A.U. after

\begin{tabular}{|c|c|c|c|c|}
\hline No. & Atom & $\mathrm{X}$ & Y & Z \\
\hline 1 & $\mathrm{C}$ & 1.973038 & -0.554560 & 0.000000 \\
\hline 2 & $\mathrm{C}$ & 1.423139 & 0.722249 & 0.000000 \\
\hline 3 & $\mathrm{C}$ & 0.000000 & 0.818093 & 0.000000 \\
\hline 4 & $\mathrm{C}$ & -0.852678 & -0.282436 & 0.000000 \\
\hline 5 & $\mathrm{C}$ & -0.273457 & -1.590880 & 0.000000 \\
\hline 6 & $\mathrm{C}$ & 1.123892 & -1.684500 & 0.000000 \\
\hline 7 & $\mathrm{H}$ & 3.048481 & -0.710395 & 0.000000 \\
\hline 8 & $\mathrm{C}$ & -0.456352 & 2.169094 & 0.000000 \\
\hline 9 & $\mathrm{C}$ & -2.263365 & -0.030659 & 0.000000 \\
\hline 10 & $\mathrm{H}$ & 1.583066 & -2.669746 & 0.000000 \\
\hline 11 & $\mathrm{C}$ & -2.708545 & 1.297613 & 0.000000 \\
\hline 12 & $\mathrm{C}$ & -1.823811 & 2.400519 & 0.000000 \\
\hline 13 & $\mathrm{H}$ & -3.779494 & 1.486830 & 0.000000 \\
\hline 14 & $\mathrm{H}$ & -2.228637 & 3.408954 & 0.000000 \\
\hline 15 & $\mathrm{C}$ & -2.652370 & -2.432427 & 0.000000 \\
\hline 16 & $\mathrm{C}$ & -4.012947 & -6.434416 & 0.000000 \\
\hline 17 & $\mathrm{C}$ & -2.657627 & -6.083256 & 0.000000 \\
\hline 18 & $\mathrm{C}$ & -5.009320 & -5.452814 & 0.000000 \\
\hline 19 & $\mathrm{H}$ & -6.055376 & -5.746624 & 0.000000 \\
\hline 20 & $\mathrm{C}$ & 1.898368 & 2.102407 & 0.000000 \\
\hline 21 & $\mathrm{C}$ & 2.600461 & 4.310454 & 0.000000 \\
\hline 22 & $\mathrm{C}$ & -1.202416 & -2.705931 & 0.000000 \\
\hline 23 & $\mathrm{C}$ & -1.017714 & -4.065953 & 0.000000 \\
\hline
\end{tabular}

\begin{tabular}{|c|c|c|c|c|}
\hline No. & Atom & $\mathrm{X}$ & Y & Z \\
\hline 24 & $\mathrm{C}$ & -2.312808 & -4.731487 & 0.000000 \\
\hline 25 & $\mathrm{C}$ & -4.671127 & -4.092121 & 0.000000 \\
\hline 26 & $\mathrm{C}$ & -3.328694 & -3.734300 & 0.000000 \\
\hline 27 & $\mathrm{C}$ & -3.155794 & -1.169248 & 0.000000 \\
\hline 28 & $\mathrm{C}$ & 0.758517 & 2.981887 & 0.000000 \\
\hline 29 & $\mathrm{C}$ & 1.181356 & 4.307788 & 0.000000 \\
\hline 30 & $\mathrm{C}$ & 3.062046 & 2.877000 & 0.000000 \\
\hline 31 & $\mathrm{C}$ & 3.389732 & 5.451150 & 0.000000 \\
\hline 32 & $\mathrm{C}$ & 4.790269 & 5.563021 & 0.000000 \\
\hline 33 & $\mathrm{H}$ & 5.177805 & 6.579418 & 0.000000 \\
\hline 34 & $\mathrm{C}$ & 4.386429 & 2.422987 & 0.000000 \\
\hline 35 & $\mathrm{C}$ & 5.568841 & 3.155962 & 0.000000 \\
\hline 36 & $\mathrm{H}$ & 6.484857 & 2.569292 & 0.000000 \\
\hline 37 & $\mathrm{C}$ & 5.744185 & 4.550484 & 0.000000 \\
\hline 38 & $\mathrm{H}$ & -4.229591 & -0.992446 & 0.000000 \\
\hline 39 & $\mathrm{H}$ & -0.065482 & -4.585319 & 0.000000 \\
\hline 40 & $\mathrm{H}$ & 0.564904 & 5.198913 & 0.000000 \\
\hline 41 & $\mathrm{H}$ & 2.843418 & 6.394022 & 0.000000 \\
\hline 42 & $\mathrm{H}$ & 6.780098 & 4.885577 & 0.000000 \\
\hline 43 & $\mathrm{H}$ & -1.890161 & -6.853342 & 0.000000 \\
\hline 44 & $\mathrm{H}$ & -4.294582 & -7.484165 & 0.000000 \\
\hline 45 & $\mathrm{H}$ & -5.452731 & -3.335917 & 0.000000 \\
\hline 46 & $\mathrm{H}$ & 4.505927 & 1.340620 & 0.000000 \\
\hline
\end{tabular}




\section{Cycloheptapentalenoindenophenalene Derivative 9b}
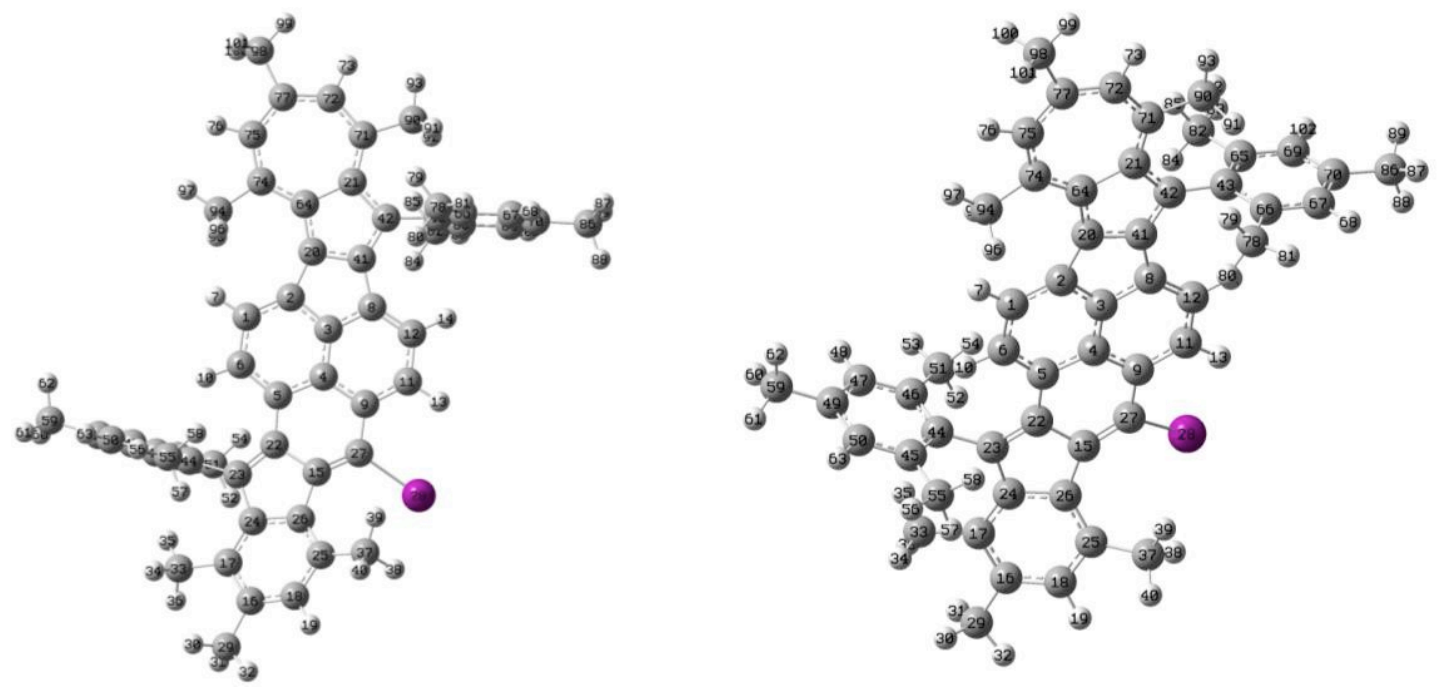

SCF Done: $\quad E(R B 3 L Y P)=-2097.43392424 \quad$ A.U. after 7 cycles

\begin{tabular}{|c|c|c|c|c|}
\hline No. & Atom & $\mathrm{X}$ & $\mathrm{Y}$ & $\mathrm{Z}$ \\
\hline 1 & $\mathrm{C}$ & -0.373370 & 2.494017 & -0.288625 \\
\hline 2 & $\mathrm{C}$ & -1.404038 & 1.559579 & -0.221283 \\
\hline 3 & $\mathrm{C}$ & -0.981681 & 0.191545 & -0.153838 \\
\hline 4 & $\mathrm{C}$ & 0.350216 & -0.236841 & -0.151163 \\
\hline 5 & $\mathrm{C}$ & 1.382117 & 0.748201 & -0.212885 \\
\hline 6 & $\mathrm{C}$ & 0.979141 & 2.086923 & -0.283231 \\
\hline 7 & $\mathrm{H}$ & -0.557265 & 3.556878 & -0.350581 \\
\hline 8 & $\mathrm{C}$ & -2.081429 & -0.703888 & -0.076976 \\
\hline 9 & $\mathrm{C}$ & 0.606562 & -1.646857 & -0.058274 \\
\hline 10 & $\mathrm{H}$ & 1.731390 & 2.862640 & -0.351172 \\
\hline 11 & $\mathrm{C}$ & -0.492126 & -2.512638 & 0.018894 \\
\hline 12 & $\mathrm{C}$ & -1.828190 & -2.063984 & 0.005872 \\
\hline 13 & $\mathrm{H}$ & -0.313952 & -3.579485 & 0.080642 \\
\hline 14 & $\mathrm{H}$ & -2.634451 & -2.788256 & 0.063422 \\
\hline 15 & $\mathrm{C}$ & 3.018766 & -1.194454 & -0.349115 \\
\hline 16 & $\mathrm{C}$ & 7.261355 & -0.965090 & -0.514949 \\
\hline 17 & $\mathrm{C}$ & 6.443402 & 0.122260 & -0.113340 \\
\hline 18 & $\mathrm{C}$ & 6.673444 & -2.128248 & -1.003249 \\
\hline 19 & $\mathrm{H}$ & 7.318915 & -2.909121 & -1.401152 \\
\hline
\end{tabular}

\begin{tabular}{|c|c|c|c|c|}
\hline No. & Atom & $\mathrm{X}$ & $\mathrm{Y}$ & $\mathrm{Z}$ \\
\hline 52 & $\mathrm{H}$ & 4.371859 & 1.104359 & 2.736773 \\
\hline 53 & $\mathrm{H}$ & 3.726710 & 2.480149 & 3.651685 \\
\hline 54 & $\mathrm{H}$ & 2.664735 & 1.514680 & 2.613965 \\
\hline 55 & $\mathrm{C}$ & 4.498887 & 2.749008 & -2.256882 \\
\hline 56 & $\mathrm{H}$ & 4.706584 & 3.570799 & -2.949384 \\
\hline 57 & $\mathrm{H}$ & 5.305205 & 2.012434 & -2.352325 \\
\hline 58 & $\mathrm{H}$ & 3.579197 & 2.251390 & -2.587597 \\
\hline 59 & $\mathrm{C}$ & 4.517540 & 6.621831 & 0.987398 \\
\hline 60 & $\mathrm{H}$ & 4.892404 & 6.818730 & 1.997638 \\
\hline 61 & $\mathrm{H}$ & 5.199568 & 7.097265 & 0.274066 \\
\hline 62 & $\mathrm{H}$ & 3.547846 & 7.130150 & 0.896767 \\
\hline 63 & $\mathrm{H}$ & 4.721996 & 5.301500 & -1.393814 \\
\hline 64 & $\mathrm{C}$ & -4.079594 & 2.280015 & -0.215025 \\
\hline 65 & $\mathrm{C}$ & -5.468571 & -1.980057 & 1.294540 \\
\hline 66 & $\mathrm{C}$ & -5.534985 & -2.119948 & -1.141375 \\
\hline 67 & $\mathrm{C}$ & -6.052912 & -3.414980 & -1.030203 \\
\hline 68 & $\mathrm{H}$ & -6.276764 & -3.970676 & -1.939146 \\
\hline 69 & $\mathrm{C}$ & -5.987182 & -3.278016 & 1.360830 \\
\hline 70 & $\mathrm{C}$ & -6.287533 & -4.014291 & 0.210985 \\
\hline
\end{tabular}




\begin{tabular}{|c|c|c|c|c|c|c|c|c|c|}
\hline 20 & $\mathrm{C}$ & -2.887106 & 1.518831 & -0.192147 & 71 & $\mathrm{C}$ & -6.607030 & 1.559338 & -0.114203 \\
\hline 21 & $\mathrm{C}$ & -5.225899 & 1.284164 & -0.129730 & 72 & $\mathrm{C}$ & -7.225639 & 2.825136 & -0.184850 \\
\hline 22 & $\mathrm{C}$ & 2.750450 & 0.260686 & -0.228468 & 73 & $\mathrm{H}$ & -8.311634 & 2.787909 & -0.158941 \\
\hline 23 & $\mathrm{C}$ & 3.950797 & 0.913214 & -0.037545 & 74 & $\mathrm{C}$ & -4.185191 & 3.690091 & -0.302133 \\
\hline 24 & $\mathrm{C}$ & 5.052186 & -0.058379 & -0.173669 & 75 & $\mathrm{C}$ & -5.345122 & 4.462654 & -0.330528 \\
\hline 25 & $\mathrm{C}$ & 5.282698 & -2.340891 & -1.037786 & 76 & $\mathrm{H}$ & -5.169738 & 5.534319 & -0.403637 \\
\hline 26 & $\mathrm{C}$ & 4.477931 & -1.330297 & -0.500912 & 77 & $\mathrm{C}$ & -6.702914 & 4.109392 & -0.281339 \\
\hline 27 & $\mathrm{C}$ & 2.002033 & -2.070839 & -0.089739 & 78 & $\mathrm{C}$ & -5.282325 & -1.525763 & -2.509639 \\
\hline 28 & I & 2.393573 & -4.088177 & 0.595082 & 79 & $\mathrm{H}$ & -5.821058 & -0.581110 & -2.651469 \\
\hline 29 & $\mathrm{C}$ & 8.768551 & -0.853212 & -0.484013 & 80 & $\mathrm{H}$ & -4.219061 & -1.302371 & -2.657243 \\
\hline 30 & $\mathrm{H}$ & 9.129792 & 0.009083 & -1.058800 & 81 & $\mathrm{H}$ & -5.595473 & -2.215879 & -3.299508 \\
\hline 31 & $\mathrm{H}$ & 9.146545 & -0.728219 & 0.539522 & 82 & $\mathrm{C}$ & -5.144981 & -1.232696 & 2.569643 \\
\hline 32 & $\mathrm{H}$ & 9.234730 & -1.751479 & -0.899959 & 83 & $\mathrm{H}$ & -5.409767 & -1.827501 & 3.449526 \\
\hline 33 & $\mathrm{C}$ & 7.112721 & 1.402160 & 0.337800 & 84 & $\mathrm{H}$ & -4.076767 & -0.993536 & 2.631303 \\
\hline 34 & $\mathrm{H}$ & 7.547113 & 1.953932 & -0.507988 & 85 & $\mathrm{H}$ & -5.681612 & -0.278238 & 2.631278 \\
\hline 35 & $\mathrm{H}$ & 6.429161 & 2.076361 & 0.849221 & 86 & $\mathrm{C}$ & -6.820554 & -5.425530 & 0.306367 \\
\hline 36 & $\mathrm{H}$ & 7.939154 & 1.181605 & 1.023310 & 87 & $\mathrm{H}$ & -7.467471 & -5.668473 & -0.543703 \\
\hline 37 & $\mathrm{C}$ & 4.783916 & -3.560384 & -1.778167 & 88 & $\mathrm{H}$ & -6.004900 & -6.161342 & 0.313146 \\
\hline 38 & $\mathrm{H}$ & 4.840657 & -4.474441 & -1.177518 & 89 & $\mathrm{H}$ & -7.398153 & -5.574818 & 1.225099 \\
\hline 39 & $\mathrm{H}$ & 3.748740 & -3.446075 & -2.106567 & 90 & $\mathrm{C}$ & -7.603050 & 0.412619 & -0.012470 \\
\hline 40 & $\mathrm{H}$ & 5.404244 & -3.716640 & -2.668823 & 91 & $\mathrm{H}$ & -7.518559 & -0.279978 & -0.854281 \\
\hline 41 & $\mathrm{C}$ & -3.264335 & 0.139481 & -0.102475 & 92 & $\mathrm{H}$ & -7.454677 & -0.180058 & 0.893861 \\
\hline 42 & $\mathrm{C}$ & -4.644985 & -0.026373 & -0.063095 & 93 & $\mathrm{H}$ & -8.626508 & 0.796181 & 0.002642 \\
\hline 43 & $\mathrm{C}$ & -5.254959 & -1.385763 & 0.031674 & 94 & $\mathrm{C}$ & -2.899896 & 4.483848 & -0.375609 \\
\hline 44 & $\mathrm{C}$ & 4.100354 & 2.371412 & 0.227416 & 95 & $\mathrm{H}$ & -2.282680 & 4.305743 & 0.510647 \\
\hline 45 & $\mathrm{C}$ & 4.369796 & 3.257841 & -0.838369 & 96 & $\mathrm{H}$ & -2.313498 & 4.194791 & -1.253638 \\
\hline 46 & $\mathrm{C}$ & 3.968643 & 2.871424 & 1.541224 & 97 & $\mathrm{H}$ & -3.096662 & 5.556981 & -0.439607 \\
\hline 47 & $\mathrm{C}$ & 4.119689 & 4.244053 & 1.765229 & 98 & $\mathrm{C}$ & -7.685271 & 5.265449 & -0.337770 \\
\hline 48 & $\mathrm{H}$ & 4.027099 & 4.622388 & 2.781661 & 99 & $\mathrm{H}$ & -8.722824 & 4.921838 & -0.306457 \\
\hline 49 & $\mathrm{C}$ & 4.387442 & 5.139075 & 0.724966 & 100 & $\mathrm{H}$ & -7.531330 & 5.952859 & 0.503727 \\
\hline 50 & $\mathrm{C}$ & 4.509278 & 4.623364 & -0.569206 & 101 & $\mathrm{H}$ & -7.550243 & 5.850875 & -1.255969 \\
\hline 51 & $\mathrm{C}$ & 3.669737 & 1.945255 & 2.698402 & 102 & $\mathrm{H}$ & -6.158660 & -3.725444 & 2.338271 \\
\hline
\end{tabular}


Bis-Azulene 13a

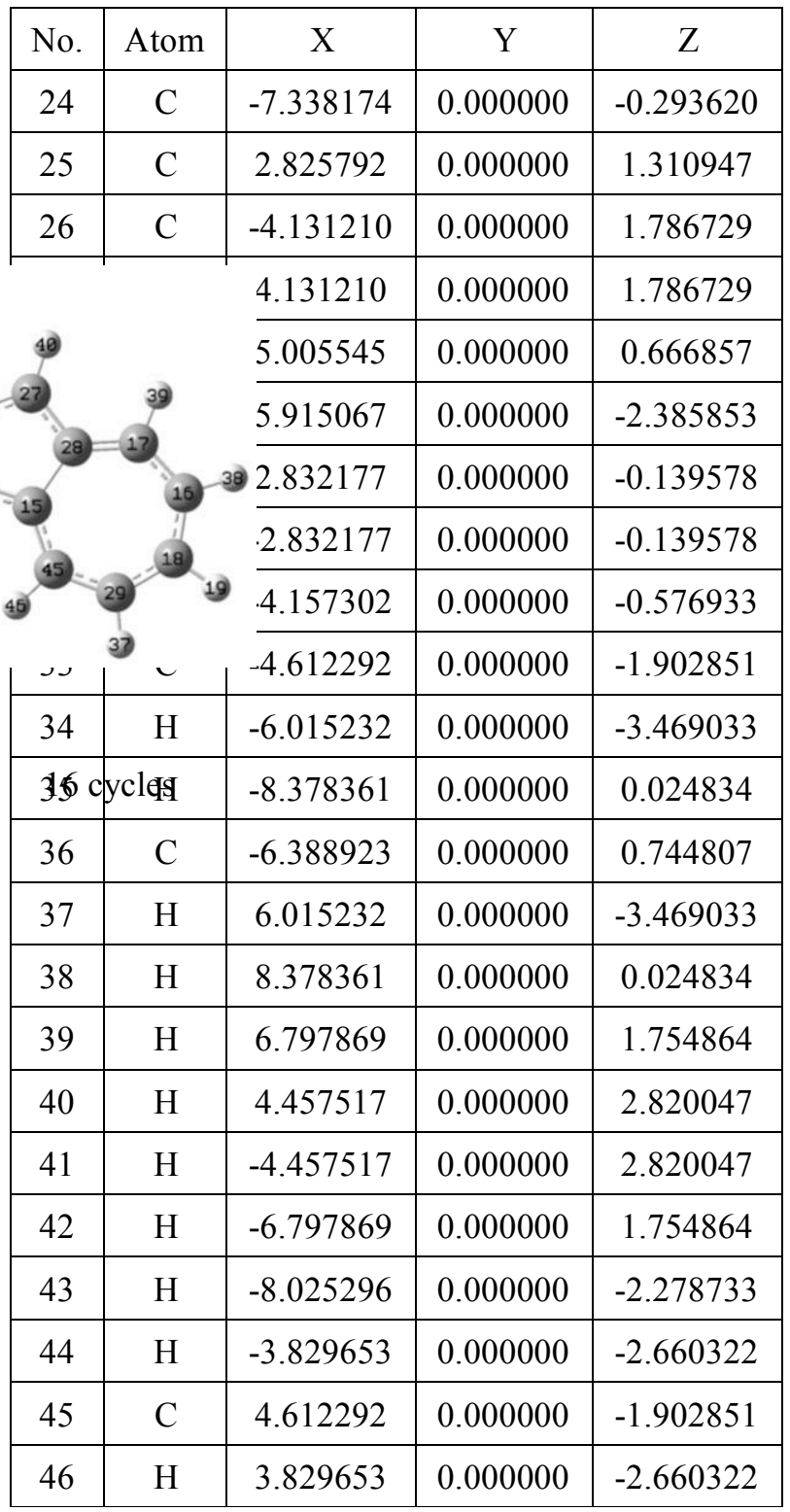

SCF Done: $\quad E(R B 3 L Y P)=-1152.76697336 \quad$ A.U. after

\begin{tabular}{|c|c|c|c|c|}
\hline No. & Atom & $X$ & Y & Z \\
\hline 1 & $\mathrm{C}$ & -0.715697 & 0.000000 & 2.974882 \\
\hline 2 & $\mathrm{C}$ & -1.432286 & 0.000000 & 1.778779 \\
\hline 3 & $\mathrm{C}$ & -0.681954 & 0.000000 & 0.576611 \\
\hline 4 & $\mathrm{C}$ & 0.681954 & 0.000000 & 0.576611 \\
\hline 5 & $\mathrm{C}$ & 1.432286 & 0.000000 & 1.778779 \\
\hline 6 & $\mathrm{C}$ & 0.715697 & 0.000000 & 2.974882 \\
\hline 7 & $\mathrm{H}$ & -1.227846 & 0.000000 & 3.933586 \\
\hline 8 & $\mathrm{C}$ & -1.447100 & 0.000000 & -0.622643 \\
\hline 9 & $\mathrm{C}$ & 1.447100 & 0.000000 & -0.622643 \\
\hline 10 & $\mathrm{H}$ & 1.227846 & 0.000000 & 3.933586 \\
\hline 11 & $\mathrm{C}$ & 0.718624 & 0.000000 & -1.809883 \\
\hline 12 & $\mathrm{C}$ & -0.718624 & 0.000000 & -1.809883 \\
\hline 13 & $\mathrm{H}$ & 1.218188 & 0.000000 & -2.775376 \\
\hline 14 & $\mathrm{H}$ & -1.218188 & 0.000000 & -2.775376 \\
\hline 15 & $\mathrm{C}$ & 4.157302 & 0.000000 & -0.576933 \\
\hline 16 & $\mathrm{C}$ & 7.338174 & 0.000000 & -0.293620 \\
\hline 17 & $\mathrm{C}$ & 6.388923 & 0.000000 & 0.744807 \\
\hline 18 & $\mathrm{C}$ & 7.124842 & 0.000000 & -1.666561 \\
\hline 19 & $\mathrm{H}$ & 8.025296 & 0.000000 & -2.278733 \\
\hline 20 & $\mathrm{C}$ & -2.825792 & 0.000000 & 1.310947 \\
\hline 21 & $\mathrm{C}$ & -5.005545 & 0.000000 & 0.666857 \\
\hline 22 & $\mathrm{C}$ & -5.915067 & 0.000000 & -2.385853 \\
\hline 23 & $\mathrm{C}$ & -7.124842 & 0.000000 & -1.666561 \\
\hline
\end{tabular}


Bis-Azulene 14a

\begin{tabular}{|c|c|c|c|c|}
\hline No. & Atom & $\mathrm{X}$ & $\mathrm{Y}$ & $\mathrm{Z}$ \\
\hline 24 & $\mathrm{C}$ & -1.078707 & -4.886787 & 0.000000 \\
\hline 25 & $\mathrm{C}$ & 1.733981 & -6.381162 & 0.000000 \\
\hline 26 & $\mathrm{C}$ & 1.517133 & -5.008466 & 0.000000 \\
\hline \multicolumn{2}{|c|}{} & .137863 & -2.915638 & 0.000000 \\
\hline
\end{tabular}

SCF Done: $\quad E($ RB3LYP $)=-1152.76800921 \quad$ A.U. after

\begin{tabular}{|c|c|c|c|c|}
\hline No. & Atom & $X$ & Y & $\mathrm{Z}$ \\
\hline 1 & $\mathrm{C}$ & -2.199910 & 1.185794 & 0.000000 \\
\hline 2 & $\mathrm{C}$ & -0.885257 & 1.656001 & 0.000000 \\
\hline 3 & $\mathrm{C}$ & 0.137863 & 0.667421 & 0.000000 \\
\hline 4 & $\mathrm{C}$ & -0.137863 & -0.667421 & 0.000000 \\
\hline 5 & $\mathrm{C}$ & -1.465589 & -1.164895 & 0.000000 \\
\hline 6 & $\mathrm{C}$ & -2.487534 & -0.218168 & 0.000000 \\
\hline 7 & $\mathrm{H}$ & -3.042095 & 1.872720 & 0.000000 \\
\hline 8 & $\mathrm{C}$ & 1.465589 & 1.164895 & 0.000000 \\
\hline 9 & $\mathrm{C}$ & 0.885257 & -1.656001 & 0.000000 \\
\hline 10 & $\mathrm{H}$ & -3.531195 & -0.522736 & 0.000000 \\
\hline 11 & $\mathrm{C}$ & 2.199910 & -1.185794 & 0.000000 \\
\hline 12 & $\mathrm{C}$ & 2.487534 & 0.218168 & 0.000000 \\
\hline 13 & $\mathrm{H}$ & 3.042095 & -1.872720 & 0.000000 \\
\hline 14 & $\mathrm{H}$ & 3.531195 & 0.522736 & 0.000000 \\
\hline 15 & $\mathrm{C}$ & 0.306704 & -4.301480 & 0.000000 \\
\hline 16 & $\mathrm{C}$ & -0.596486 & -7.363217 & 0.000000 \\
\hline 17 & $\mathrm{C}$ & -1.427218 & -6.229049 & 0.000000 \\
\hline 18 & $\mathrm{C}$ & 0.792182 & -7.426066 & 0.000000 \\
\hline 19 & $\mathrm{H}$ & 1.214780 & -8.429425 & 0.000000 \\
\hline 20 & $\mathrm{C}$ & -0.137863 & 2.915638 & 0.000000 \\
\hline 21 & $\mathrm{C}$ & 1.078707 & 4.886787 & 0.000000 \\
\hline 22 & $\mathrm{C}$ & -1.283741 & -2.621604 & 0.000000 \\
\hline 23 & $\mathrm{C}$ & -2.004875 & -3.809350 & 0.000000 \\
\hline
\end{tabular}




\section{Azulenoacenaphthylene Derivative 11}

\begin{tabular}{|c|c|c|c|c|}
\hline No. & Atom & $\mathrm{X}$ & Y & Z \\
\hline 52 & $\mathrm{H}$ & -4.204066 & -4.532130 & -0.981202 \\
\hline 53 & $\mathrm{H}$ & -5.712392 & -4.664953 & -1.906608 \\
\hline 54 & $\mathrm{H}$ & -4.629260 & -3.278096 & -2.136346 \\
\hline & $r$ & -8.630601 & 3.840083 & -1.073270 \\
\hline & I & -8.698046 & 4.880633 & -0.738319 \\
\hline & I & -8.946471 & 3.799682 & -2.121447 \\
\hline & I & -9.363317 & 3.263247 & -0.492432 \\
\hline & : & -9.329696 & -3.024821 & 1.121030 \\
\hline & I & -9.601422 & -4.057146 & 0.874959 \\
\hline & I & -9.630708 & -2.832439 & 2.156416 \\
\hline & I & -9.934949 & -2.369163 & 0.480065 \\
\hline & : & -4.209675 & 3.854219 & 1.373145 \\
\hline & I & -3.271994 & 4.236331 & 0.951448 \\
\hline & I & -4.704415 & 4.674404 & 1.902477 \\
\hline & I & -3.931194 & 3.090961 & 2.109773 \\
\hline & : & -5.093549 & -0.588389 & 2.406560 \\
\hline 68 & $\mathrm{H}$ & -5.788308 & -0.114250 & 3.106690 \\
\hline
\end{tabular}

SCF Done: $\quad E(R B 3 L Y P)=-2086.60894281$

A.U. after

2 cycles

\begin{tabular}{|c|c|c|c|c|}
\hline No. & Atom & $\mathrm{X}$ & $\mathrm{Y}$ & $\mathrm{Z}$ \\
\hline 1 & $\mathrm{C}$ & 1.446452 & 1.753833 & 0.059131 \\
\hline 2 & $\mathrm{C}$ & 1.946593 & 0.465107 & 0.000570 \\
\hline 3 & $\mathrm{C}$ & 1.044906 & -0.633973 & -0.059479 \\
\hline 4 & $\mathrm{C}$ & -0.357450 & -0.487691 & -0.048713 \\
\hline 5 & $\mathrm{C}$ & -0.857585 & 0.865237 & -0.044065 \\
\hline 6 & $\mathrm{C}$ & 0.052719 & 1.931239 & 0.025497 \\
\hline 7 & $\mathrm{H}$ & 2.101909 & 2.617164 & 0.112101 \\
\hline 8 & $\mathrm{C}$ & 1.758049 & -1.883044 & -0.118978 \\
\hline 9 & $\mathrm{C}$ & -1.127347 & -1.706123 & -0.038146 \\
\hline 10 & $\mathrm{H}$ & -0.352325 & 2.938223 & 0.032174 \\
\hline 11 & $\mathrm{C}$ & -0.433738 & -2.918084 & -0.103454 \\
\hline 12 & $\mathrm{C}$ & 0.970415 & -3.025444 & -0.151179 \\
\hline 13 & $\mathrm{H}$ & -1.018645 & -3.832728 & -0.092941 \\
\hline 14 & $\mathrm{H}$ & 1.385265 & -4.021627 & -0.191200 \\
\hline 15 & $\mathrm{C}$ & 3.278413 & -0.112685 & -0.014007 \\
\hline 16 & $\mathrm{C}$ & 5.435326 & -0.819677 & -0.016980 \\
\hline 17 & $\mathrm{C}$ & 6.225123 & -3.892771 & -0.294133 \\
\hline
\end{tabular}




\begin{tabular}{|c|c|c|c|c|}
\hline 18 & $\mathrm{C}$ & 7.474308 & -3.270736 & -0.161456 \\
\hline 19 & $\mathrm{C}$ & 7.712953 & -1.909499 & -0.004103 \\
\hline 20 & $\mathrm{C}$ & 4.594049 & 0.339093 & 0.039135 \\
\hline 21 & $\mathrm{C}$ & 3.198242 & -1.535884 & -0.104963 \\
\hline 22 & $\mathrm{C}$ & 4.524834 & -2.032681 & -0.132815 \\
\hline 23 & $\mathrm{C}$ & 4.927086 & -3.381483 & -0.280547 \\
\hline 24 & $\mathrm{H}$ & 6.280612 & -4.972128 & -0.422609 \\
\hline 25 & $\mathrm{H}$ & 8.765102 & -1.650206 & 0.080678 \\
\hline 26 & $\mathrm{C}$ & 6.843473 & -0.802735 & 0.052547 \\
\hline 27 & $\mathrm{C}$ & -2.233940 & 1.211161 & -0.165776 \\
\hline 28 & $\mathrm{C}$ & -2.542485 & -1.774330 & 0.103402 \\
\hline 29 & $\mathrm{C}$ & -3.345573 & 1.684383 & -0.329688 \\
\hline 30 & $\mathrm{C}$ & -3.724407 & -2.012992 & 0.284352 \\
\hline 31 & $\mathrm{C}$ & -4.654800 & 2.216618 & -0.518167 \\
\hline 32 & $\mathrm{C}$ & -5.103304 & 3.283999 & 0.299491 \\
\hline 33 & $\mathrm{C}$ & -5.496515 & 1.694281 & -1.532200 \\
\hline 34 & $\mathrm{C}$ & -6.384029 & 3.797282 & 0.093596 \\
\hline 35 & $\mathrm{C}$ & -6.768015 & 2.243397 & -1.696512 \\
\hline 36 & $\mathrm{C}$ & -7.235409 & 3.290026 & -0.893749 \\
\hline 37 & $\mathrm{H}$ & -6.727642 & 4.617002 & 0.721608 \\
\hline 38 & $\mathrm{H}$ & -7.412961 & 1.843137 & -2.476464 \\
\hline 39 & $\mathrm{C}$ & -5.111970 & -2.264171 & 0.496761 \\
\hline 40 & $\mathrm{C}$ & -5.786248 & -3.217552 & -0.306878 \\
\hline 41 & $\mathrm{C}$ & -5.810180 & -1.576722 & 1.520865 \\
\hline 42 & $\mathrm{C}$ & -7.141735 & -3.453567 & -0.077978 \\
\hline 43 & $\mathrm{C}$ & -7.165842 & -1.848258 & 1.707249 \\
\hline 44 & $\mathrm{C}$ & -7.853627 & -2.776821 & 0.918629 \\
\hline 45 & $\mathrm{H}$ & -7.657386 & -4.186518 & -0.695756 \\
\hline 46 & $\mathrm{H}$ & -7.700923 & -1.320974 & 2.494641 \\
\hline 47 & $\mathrm{C}$ & -5.016276 & 0.581233 & -2.429644 \\
\hline 48 & $\mathrm{H}$ & -5.805415 & 0.267607 & -3.119858 \\
\hline 49 & $\mathrm{H}$ & -4.149465 & 0.897601 & -3.023261 \\
\hline 50 & $\mathrm{H}$ & -4.696361 & -0.290306 & -1.848567 \\
\hline 51 & $\mathrm{C}$ & -5.048564 & -3.964461 & -1.390697 \\
\hline
\end{tabular}

\begin{tabular}{|c|c|c|c|c|}
\hline 69 & $\mathrm{H}$ & -4.304432 & -1.080014 & 2.989191 \\
\hline 70 & $\mathrm{H}$ & -4.605228 & 0.195036 & 1.817388 \\
\hline 71 & $\mathrm{C}$ & 3.844465 & -4.424458 & -0.447732 \\
\hline 72 & $\mathrm{H}$ & 3.246364 & -4.511244 & 0.465804 \\
\hline 73 & $\mathrm{H}$ & 3.169338 & -4.160329 & -1.266616 \\
\hline 74 & $\mathrm{H}$ & 4.269314 & -5.408262 & -0.662060 \\
\hline 75 & $\mathrm{C}$ & 7.572916 & 0.523240 & 0.215747 \\
\hline 76 & $\mathrm{H}$ & 7.388293 & 1.199839 & -0.622924 \\
\hline 77 & $\mathrm{H}$ & 7.259465 & 1.054086 & 1.118273 \\
\hline 78 & $\mathrm{H}$ & 8.651627 & 0.359934 & 0.281399 \\
\hline 79 & C & 8.678501 & -4.194015 & -0.203885 \\
\hline 80 & $\mathrm{H}$ & 8.616339 & -4.958097 & 0.580921 \\
\hline 81 & $\mathrm{H}$ & 8.731735 & -4.725533 & -1.162516 \\
\hline 82 & $\mathrm{H}$ & 9.616363 & -3.648259 & -0.069825 \\
\hline 83 & $\mathrm{C}$ & 4.906394 & 1.796748 & 0.125377 \\
\hline 84 & $\mathrm{C}$ & 5.078695 & 2.554273 & -1.053133 \\
\hline 85 & $\mathrm{C}$ & 4.927355 & 2.444531 & 1.379836 \\
\hline 86 & $\mathrm{C}$ & 5.302642 & 3.931884 & -0.954673 \\
\hline 87 & $\mathrm{C}$ & 5.154025 & 3.823683 & 1.433511 \\
\hline 88 & $\mathrm{C}$ & 5.351067 & 4.586980 & 0.278885 \\
\hline 89 & $\mathrm{H}$ & 5.437008 & 4.508372 & -1.868205 \\
\hline 90 & $\mathrm{H}$ & 5.173505 & 4.314847 & 2.404879 \\
\hline 91 & $\mathrm{C}$ & 5.012087 & 1.898845 & -2.414739 \\
\hline 92 & $\mathrm{H}$ & 5.746024 & 1.090466 & -2.515484 \\
\hline 93 & $\mathrm{H}$ & 4.028377 & 1.448701 & -2.592854 \\
\hline 94 & $\mathrm{H}$ & 5.199716 & 2.627691 & -3.209445 \\
\hline 95 & $\mathrm{C}$ & 4.699553 & 1.670397 & 2.659353 \\
\hline 96 & $\mathrm{H}$ & 3.685130 & 1.256075 & 2.700750 \\
\hline 97 & $\mathrm{H}$ & 5.385834 & 0.820317 & 2.749934 \\
\hline 98 & $\mathrm{H}$ & 4.836901 & 2.312902 & 3.534796 \\
\hline 99 & $\mathrm{C}$ & 5.627642 & 6.070169 & 0.364630 \\
\hline 100 & $\mathrm{H}$ & 6.699319 & 6.269307 & 0.500712 \\
\hline 101 & $\mathrm{H}$ & 5.314641 & 6.589897 & -0.547208 \\
\hline 102 & $\mathrm{H}$ & 5.104829 & 6.527707 & 1.211728 \\
\hline
\end{tabular}




\section{Vinyl Cation Intermediate 16a}

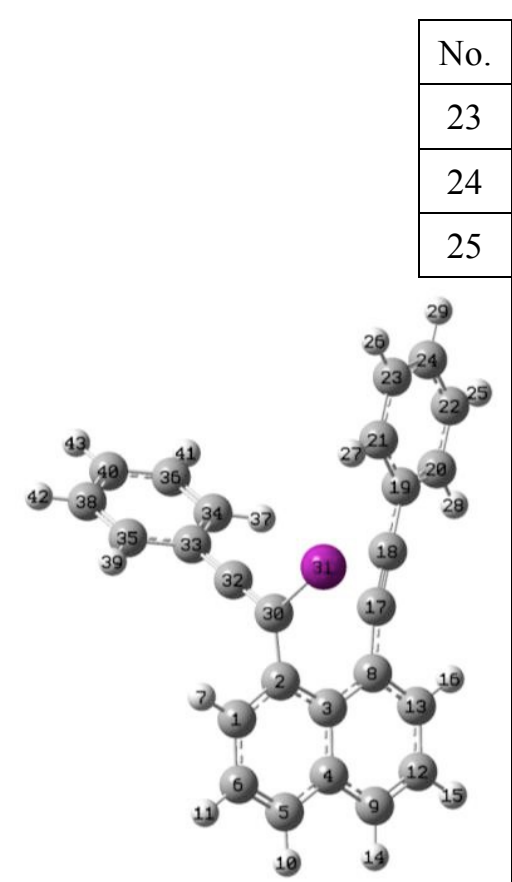

\begin{tabular}{|c|c|c|c|c|}
\hline No. & Atom & $X$ & $\mathrm{Y}$ & $\mathrm{Z}$ \\
\hline 23 & $\mathrm{C}$ & 4.573356 & -1.271314 & 0.878575 \\
\hline 24 & $\mathrm{C}$ & 5.270725 & -2.268848 & 0.190180 \\
\hline 25 & $\mathrm{H}$ & 5.128973 & -3.898820 & -1.216714 \\
\hline & $\mathrm{H}$ & 5.100094 & -0.614715 & 1.565020 \\
\hline & $\mathrm{H}$ & 2.656388 & -0.356782 & 1.240515 \\
\hline & $\mathrm{H}$ & 2.685492 & -3.641474 & -1.549844 \\
\hline 8 & $\mathrm{H}$ & 6.340226 & -2.385004 & 0.338897 \\
\hline & $\mathrm{C}$ & -0.892890 & 0.898042 & 0.480200 \\
\hline & I & -0.320754 & 0.541967 & 2.565190 \\
\hline & $\mathrm{C}$ & -0.097837 & 1.628207 & -0.214019 \\
\hline & $\mathrm{C}$ & 0.773648 & 2.350605 & -0.991601 \\
\hline & $\mathrm{C}$ & 1.757375 & 1.667723 & -1.776248 \\
\hline נג & $\mathrm{C}$ & 0.704183 & 3.779866 & -1.020503 \\
\hline \multicolumn{2}{|c|}{36 dyclę } & 2.635226 & 2.400088 & -2.553547 \\
\hline 37 & $\mathrm{H}$ & 1.791955 & 0.583896 & -1.745653 \\
\hline 38 & $\mathrm{C}$ & 1.594804 & 4.490049 & -1.802675 \\
\hline 39 & $\mathrm{H}$ & -0.044932 & 4.285372 & -0.420164 \\
\hline 40 & $\mathrm{C}$ & 2.554207 & 3.802666 & -2.566530 \\
\hline 41 & $\mathrm{H}$ & 3.384886 & 1.894595 & -3.153368 \\
\hline 42 & $\mathrm{H}$ & 1.555062 & 5.573979 & -1.830166 \\
\hline 43 & $\mathrm{H}$ & 3.247652 & 4.369371 & -3.181512 \\
\hline & & & & \\
\hline
\end{tabular}

SCF Done: $\mathrm{E}(\mathrm{RB} 3 \mathrm{LYP})=-1011.41945883$
\begin{tabular}{|c|c|c|c|c|}
\hline No. & Atom & $\mathrm{X}$ & $\mathrm{Y}$ & A.U. after \\
\hline 1 & $\mathrm{C}$ & -3.220715 & 1.436133 & 0.065720 \\
\hline 2 & $\mathrm{C}$ & -2.208710 & 0.479826 & -0.046321 \\
\hline 3 & $\mathrm{C}$ & -2.467754 & -0.806169 & -0.628655 \\
\hline 4 & $\mathrm{C}$ & -3.824199 & -1.067907 & -1.036094 \\
\hline 5 & $\mathrm{C}$ & -4.824570 & -0.074346 & -0.877792 \\
\hline 6 & $\mathrm{C}$ & -4.533214 & 1.162624 & -0.341982 \\
\hline 7 & $\mathrm{H}$ & -2.983713 & 2.399984 & 0.504952 \\
\hline 8 & $\mathrm{C}$ & -1.489748 & -1.837014 & -0.850426 \\
\hline 9 & $\mathrm{C}$ & -4.176478 & -2.318938 & -1.602366 \\
\hline 10 & $\mathrm{H}$ & -5.836684 & -0.307512 & -1.196727 \\
\hline 11 & $\mathrm{H}$ & -5.305006 & 1.916846 & -0.229429 \\
\hline 12 & $\mathrm{C}$ & -3.225308 & -3.298867 & -1.789291 \\
\hline 13 & $\mathrm{C}$ & -1.895931 & -3.051610 & -1.422716 \\
\hline 14 & $\mathrm{H}$ & -5.211554 & -2.489878 & -1.883554 \\
\hline 15 & $\mathrm{H}$ & -3.494992 & -4.257240 & -2.221385 \\
\hline 16 & $\mathrm{H}$ & -1.143284 & -3.816851 & -1.579553 \\
\hline 17 & $\mathrm{C}$ & -0.101084 & -1.726560 & -0.559724 \\
\hline 18 & $\mathrm{C}$ & 1.100829 & -1.842072 & -0.384399 \\
\hline 19 & $\mathrm{C}$ & 2.505245 & -1.974831 & -0.185410 \\
\hline 20 & $\mathrm{C}$ & 3.218550 & -2.979542 & -0.874591 \\
\hline 21 & $\mathrm{C}$ & 3.201963 & -1.120521 & 0.695474 \\
\hline 22 & $\mathrm{C}$ & 4.589890 & -3.120622 & -0.684655 \\
\hline
\end{tabular}




\section{Vinyl Cation Intermediate $16 \mathrm{~b}$}

SCF Done: $\quad E(R B 3 L Y P)=-1247.33267600 \quad$ A.U. after

\begin{tabular}{|c|c|c|c|c|}
\hline No. & Atom & $\mathrm{X}$ & $\mathrm{Y}$ & $\mathrm{Z}$ \\
\hline 1 & $\mathrm{C}$ & -3.787515 & -0.693238 & -0.397204 \\
\hline 2 & $\mathrm{C}$ & -2.429225 & -0.999921 & -0.306975 \\
\hline 3 & $\mathrm{C}$ & -1.922035 & -2.240669 & -0.823004 \\
\hline 4 & $\mathrm{C}$ & -2.891404 & -3.145423 & -1.390894 \\
\hline 5 & $\mathrm{C}$ & -4.263457 & -2.787140 & -1.454672 \\
\hline 6 & $\mathrm{C}$ & -4.710661 & -1.578036 & -0.974899 \\
\hline 7 & $\mathrm{H}$ & -4.140372 & 0.239300 & 0.028423 \\
\hline 8 & $\mathrm{C}$ & -0.542822 & -2.650230 & -0.833589 \\
\hline 9 & $\mathrm{C}$ & -2.492882 & -4.408234 & -1.895994 \\
\hline 10 & $\mathrm{H}$ & -4.960203 & -3.496182 & -1.893164 \\
\hline 11 & $\mathrm{H}$ & -5.761338 & -1.311075 & -1.023305 \\
\hline 12 & $\mathrm{C}$ & -1.171002 & -4.790607 & -1.866576 \\
\hline 13 & $\mathrm{C}$ & -0.210979 & -3.910540 & -1.348451 \\
\hline 14 & $\mathrm{H}$ & -3.253426 & -5.067992 & -2.303623 \\
\hline 15 & $\mathrm{H}$ & -0.863864 & -5.759510 & -2.247515 \\
\hline 16 & $\mathrm{H}$ & 0.833822 & -4.201342 & -1.341109 \\
\hline 17 & $\mathrm{C}$ & 0.575421 & -1.862079 & -0.417382 \\
\hline 18 & $\mathrm{C}$ & 1.716182 & -1.479398 & -0.198468 \\
\hline 19 & $\mathrm{C}$ & 3.064305 & -1.030970 & -0.068014 \\
\hline
\end{tabular}

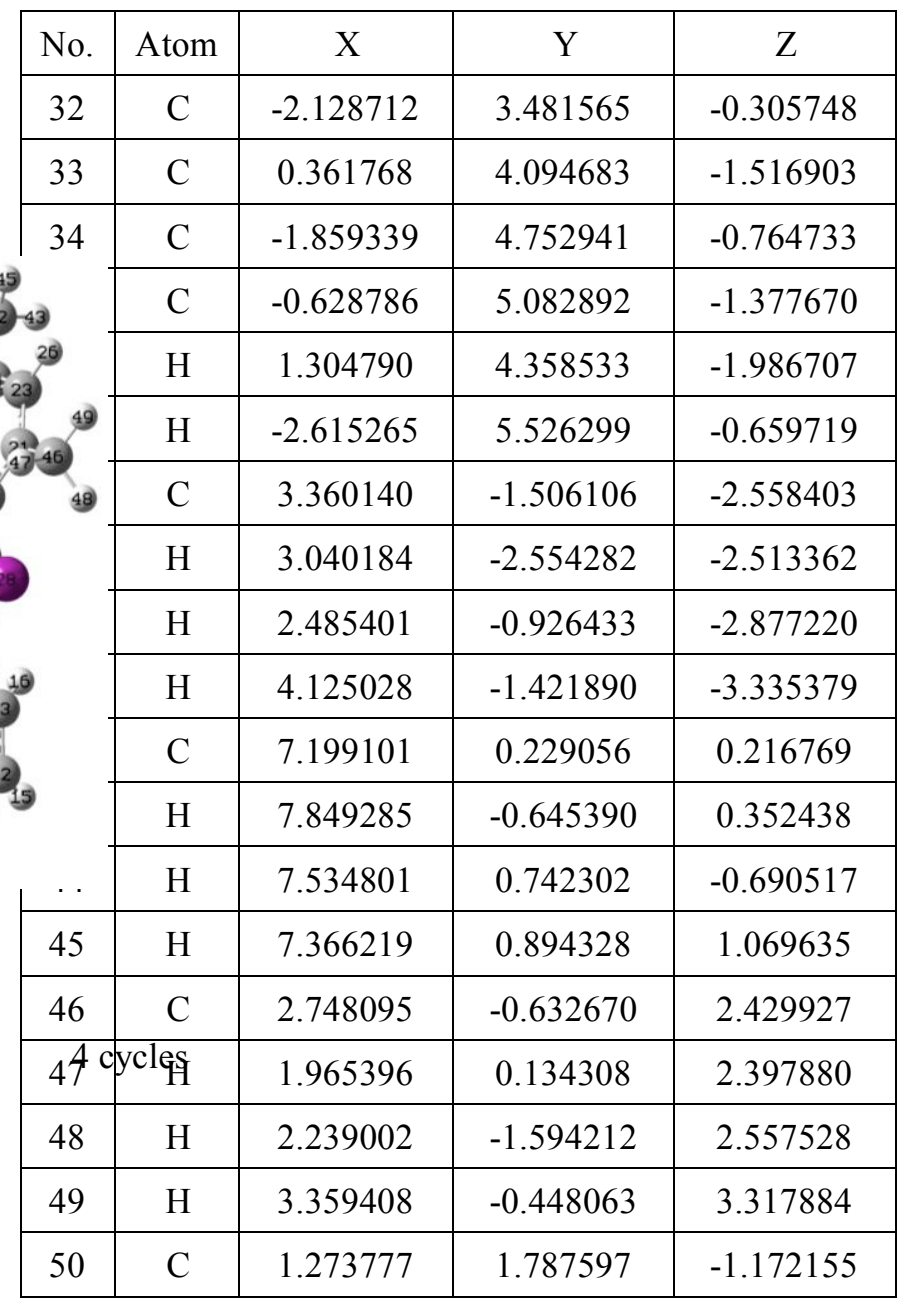




\begin{tabular}{|l|l|l|l|l|}
\hline 20 & $\mathrm{C}$ & 3.891715 & -1.037006 & -1.226004 \\
\hline 21 & $\mathrm{C}$ & 3.593564 & -0.616973 & 1.181516 \\
\hline 22 & $\mathrm{C}$ & 5.212692 & -0.608943 & -1.108794 \\
\hline 23 & $\mathrm{C}$ & 4.924648 & -0.202675 & 1.241870 \\
\hline 24 & $\mathrm{C}$ & 5.752945 & -0.189338 & 0.114028 \\
\hline 25 & $\mathrm{H}$ & 5.844032 & -0.610927 & -1.994536 \\
\hline 26 & $\mathrm{H}$ & 5.331064 & 0.109891 & 2.201022 \\
\hline 27 & $\mathrm{C}$ & -1.630243 & 0.014620 & 0.408506 \\
\hline 28 & $\mathrm{I}$ & -1.233225 & -0.252242 & 2.563158 \\
\hline 29 & $\mathrm{C}$ & -1.344189 & 1.196179 & -0.019370 \\
\hline 30 & $\mathrm{C}$ & -1.096939 & 2.464402 & -0.452631 \\
\hline 31 & $\mathrm{C}$ & 0.177340 & 2.800146 & -1.061682 \\
\hline
\end{tabular}

\begin{tabular}{|c|c|c|c|c|}
\hline 51 & $\mathrm{H}$ & 1.769785 & 1.651510 & -0.201927 \\
\hline 52 & $\mathrm{H}$ & 0.902840 & 0.801370 & -1.464437 \\
\hline 53 & $\mathrm{H}$ & 2.034641 & 2.112276 & -1.886012 \\
\hline 54 & $\mathrm{C}$ & -0.387033 & 6.484231 & -1.854179 \\
\hline 55 & $\mathrm{H}$ & -1.235749 & 6.850534 & -2.443261 \\
\hline 56 & $\mathrm{H}$ & -0.285895 & 7.160893 & -0.994398 \\
\hline 57 & $\mathrm{H}$ & 0.521330 & 6.563359 & -2.455838 \\
\hline 58 & $\mathrm{C}$ & -3.456134 & 3.157061 & 0.319881 \\
\hline 59 & $\mathrm{H}$ & -4.045363 & 2.506540 & -0.338199 \\
\hline 60 & $\mathrm{H}$ & -3.339083 & 2.636659 & 1.276533 \\
\hline 61 & $\mathrm{H}$ & -4.035526 & 4.066980 & 0.492884 \\
\hline & & & & \\
\hline
\end{tabular}


Vinyl Cation Intermediate 16c

\begin{tabular}{|c|c|c|c|c|c|}
\hline \multicolumn{6}{|c|}{ Atom } \\
\hline & 53 & $\mathrm{H}$ & 0.910449 & 3.008319 & 1.315648 \\
\hline & 54 & $\mathrm{H}$ & 6.709574 & 2.595018 & -2.703655 \\
\hline & 55 & $\mathrm{C}$ & 5.863597 & -2.286862 & 1.592932 \\
\hline & & $\mathrm{H}$ & 6.775235 & -2.480224 & 2.165491 \\
\hline 9 & & $\mathrm{H}$ & 5.676190 & -1.207567 & 1.610386 \\
\hline $9^{37}$ & & $\mathrm{H}$ & 5.023606 & -2.753448 & 2.120197 \\
\hline 52- (3) & & $\mathrm{C}$ & 3.880150 & -3.199401 & -3.013618 \\
\hline 6) 63 & & $\mathrm{H}$ & 3.004433 & -3.740379 & -2.634709 \\
\hline & & $\mathrm{H}$ & 3.555316 & -2.167938 & -3.197091 \\
\hline & & $\mathrm{H}$ & 4.159529 & -3.642833 & -3.973475 \\
\hline & & $\mathrm{C}$ & 3.419630 & 4.605266 & 1.105315 \\
\hline & & $\mathrm{H}$ & 2.384908 & 4.638560 & 0.742025 \\
\hline & & $\mathrm{H}$ & 3.426041 & 4.009857 & 2.024403 \\
\hline & & $\mathrm{H}$ & 3.714695 & 5.626971 & 1.356761 \\
\hline & & $\mathrm{C}$ & 5.196616 & 0.637848 & -1.639323 \\
\hline & & $\mathrm{H}$ & 5.720504 & 0.462900 & -2.582400 \\
\hline 729829 & $49 y$ & lesH & 5.717439 & 0.064449 & -0.861906 \\
\hline $\mathrm{V}$ & 70 & $\mathrm{H}$ & 4.189678 & 0.216364 & -1.706076 \\
\hline
\end{tabular}

SCF Done: $\quad \mathrm{E}(\mathrm{RB} 3 \mathrm{LYP})=-2097.64729829$

\begin{tabular}{|c|c|c|c|c|}
\hline No. & Atom & $\mathrm{X}$ & $\mathrm{Y}$ & $\mathrm{Z}$ \\
\hline 1 & $\mathrm{C}$ & -7.732067 & -2.143088 & 1.586551 \\
\hline 2 & $\mathrm{C}$ & -8.239590 & -3.245538 & 0.886341 \\
\hline 3 & $\mathrm{C}$ & -6.426252 & -1.690650 & 1.409818 \\
\hline 4 & $\mathrm{C}$ & -7.396972 & -3.904764 & -0.017660 \\
\hline 5 & $\mathrm{C}$ & -5.590230 & -2.373877 & 0.486340 \\
\hline 6 & $\mathrm{C}$ & -6.081029 & -3.499166 & -0.230157 \\
\hline 7 & $\mathrm{C}$ & 7.175784 & -3.406959 & -0.241401 \\
\hline 8 & $\mathrm{C}$ & -4.251159 & -1.949803 & 0.279316 \\
\hline 9 & $\mathrm{C}$ & 5.988560 & -2.811305 & 0.185451 \\
\hline 10 & $\mathrm{C}$ & -0.982967 & -2.591375 & -0.356793 \\
\hline 11 & $\mathrm{C}$ & 7.325522 & -3.929956 & -1.531010 \\
\hline 12 & $\mathrm{C}$ & -3.105035 & -1.572758 & 0.084405 \\
\hline 13 & $\mathrm{C}$ & 0.401240 & -2.591421 & -0.377625 \\
\hline 14 & $\mathrm{C}$ & 4.902195 & -2.728130 & -0.723788 \\
\hline 15 & $\mathrm{C}$ & -1.703540 & -1.425731 & -0.062976 \\
\hline 16 & $\mathrm{C}$ & 3.658335 & -2.140413 & -0.349536 \\
\hline 17 & $\mathrm{C}$ & 6.239333 & -3.841807 & -2.411017 \\
\hline 18 & $\mathrm{C}$ & -7.151023 & 3.789845 & -0.751376 \\
\hline
\end{tabular}




\begin{tabular}{|c|c|c|c|c|}
\hline 19 & $\mathrm{C}$ & 5.029128 & -3.260145 & -2.037030 \\
\hline 20 & $\mathrm{C}$ & 2.564489 & -1.637431 & -0.129774 \\
\hline 21 & $\mathrm{C}$ & 1.153490 & -1.454159 & -0.039487 \\
\hline 22 & $\mathrm{C}$ & -5.911647 & 3.332793 & -0.309518 \\
\hline 23 & $\mathrm{C}$ & -7.851970 & 3.152063 & -1.783082 \\
\hline 24 & $\mathrm{C}$ & -0.981756 & -0.203899 & 0.167365 \\
\hline 25 & $\mathrm{C}$ & 0.462756 & -0.236266 & 0.283846 \\
\hline 26 & $\mathrm{C}$ & -5.355193 & 2.174907 & -0.920106 \\
\hline 27 & $\mathrm{C}$ & -3.019011 & 1.265563 & -0.092807 \\
\hline 28 & $\mathrm{C}$ & -7.280205 & 2.017387 & -2.376213 \\
\hline 29 & $\mathrm{C}$ & -4.096419 & 1.696459 & -0.480423 \\
\hline 30 & $\mathrm{C}$ & -1.673508 & 1.055016 & 0.285254 \\
\hline 31 & $\mathrm{C}$ & -6.048292 & 1.511530 & -1.970806 \\
\hline 32 & $\mathrm{C}$ & 1.129920 & 0.961996 & 0.713329 \\
\hline 33 & $\mathrm{C}$ & 2.559087 & 1.086452 & 1.011138 \\
\hline 34 & $\mathrm{C}$ & 4.332964 & 4.030565 & 0.057485 \\
\hline 35 & $\mathrm{C}$ & -0.969737 & 2.189079 & 0.715768 \\
\hline 36 & $\mathrm{C}$ & 0.393474 & 2.129597 & 0.948725 \\
\hline 37 & $\mathrm{C}$ & 5.236192 & 4.839084 & -0.602304 \\
\hline 38 & $\mathrm{C}$ & 3.403078 & 1.801288 & 0.359755 \\
\hline 39 & $\mathrm{C}$ & 4.291260 & 2.620221 & -0.285739 \\
\hline 40 & $\mathrm{C}$ & 6.094477 & 4.340865 & -1.606237 \\
\hline 41 & $\mathrm{C}$ & 5.182146 & 2.096326 & -1.298331 \\
\hline 42 & $\mathrm{C}$ & 6.043928 & 2.976333 & -1.934501 \\
\hline 43 & $\mathrm{H}$ & -8.375018 & -1.623403 & 2.293349 \\
\hline 44 & $\mathrm{H}$ & -7.778248 & -4.760196 & -0.570614 \\
\hline 45 & $\mathrm{H}$ & 8.006934 & -3.472917 & 0.457074 \\
\hline 46 & $\mathrm{H}$ & -1.528965 & -3.509218 & -0.545152 \\
\hline 47 & $\mathrm{H}$ & 0.934590 & -3.503205 & -0.622568 \\
\hline 48 & $\mathrm{H}$ & -7.585047 & 4.668056 & -0.279228 \\
\hline 49 & $\mathrm{H}$ & 6.338212 & -4.243273 & -3.417110 \\
\hline 50 & $\mathrm{H}$ & -7.814005 & 1.514108 & -3.179051 \\
\hline 51 & $\mathrm{H}$ & 5.286492 & 5.894001 & -0.345789 \\
\hline 52 & $\mathrm{H}$ & -1.505625 & 3.123422 & 0.839528 \\
\hline
\end{tabular}

\begin{tabular}{|c|c|c|c|c|}
\hline 71 & $\mathrm{C}$ & -5.199974 & -4.238337 & -1.206982 \\
\hline 72 & $\mathrm{H}$ & -4.834146 & -3.577022 & -2.001630 \\
\hline 73 & $\mathrm{H}$ & -4.316116 & -4.655707 & -0.708555 \\
\hline 74 & $\mathrm{H}$ & -5.742715 & -5.063804 & -1.676039 \\
\hline 75 & $\mathrm{C}$ & -5.909637 & -0.512543 & 2.197415 \\
\hline 76 & $\mathrm{H}$ & -5.572972 & 0.294037 & 1.537316 \\
\hline 77 & $\mathrm{H}$ & -6.686405 & -0.114545 & 2.856622 \\
\hline 78 & $\mathrm{H}$ & -5.050222 & -0.795197 & 2.817499 \\
\hline 79 & $\mathrm{C}$ & -5.453879 & 0.301937 & -2.646963 \\
\hline 80 & $\mathrm{H}$ & -5.234486 & -0.492414 & -1.926200 \\
\hline 81 & $\mathrm{H}$ & -6.136852 & -0.096673 & -3.402434 \\
\hline 82 & $\mathrm{H}$ & -4.507815 & 0.550803 & -3.143520 \\
\hline 83 & $\mathrm{C}$ & -5.184439 & 4.046878 & 0.802906 \\
\hline 84 & $\mathrm{H}$ & -5.014212 & 3.387178 & 1.662220 \\
\hline 85 & $\mathrm{H}$ & -4.199929 & 4.400562 & 0.472101 \\
\hline 86 & $\mathrm{H}$ & -5.754884 & 4.913203 & 1.149150 \\
\hline 87 & $\mathrm{C}$ & 7.039885 & 5.270361 & -2.311418 \\
\hline 88 & $\mathrm{H}$ & 7.726995 & 4.733859 & -2.970214 \\
\hline 89 & $\mathrm{H}$ & 6.480961 & 5.994442 & -2.919205 \\
\hline 90 & $\mathrm{H}$ & 7.627652 & 5.851432 & -1.590913 \\
\hline 91 & $\mathrm{C}$ & 8.609908 & -4.601335 & -1.951758 \\
\hline 92 & $\mathrm{H}$ & 9.471789 & -4.194514 & -1.413135 \\
\hline 93 & $\mathrm{H}$ & 8.574360 & -5.678697 & -1.741961 \\
\hline 94 & $\mathrm{H}$ & 8.790694 & -4.486773 & -3.025549 \\
\hline 95 & $\mathrm{C}$ & -9.177751 & 3.686185 & -2.264703 \\
\hline 96 & $\mathrm{H}$ & -9.045342 & 4.312995 & -3.156852 \\
\hline 97 & $\mathrm{H}$ & -9.860404 & 2.875127 & -2.539711 \\
\hline 98 & $\mathrm{H}$ & -9.664992 & 4.301618 & -1.502201 \\
\hline 99 & $\mathrm{C}$ & -9.649233 & -3.728993 & 1.122879 \\
\hline 100 & $\mathrm{H}$ & -9.681509 & -4.450792 & 1.950126 \\
\hline 101 & $\mathrm{H}$ & -10.318226 & -2.903553 & 1.387315 \\
\hline 102 & $\mathrm{H}$ & -10.055649 & -4.230553 & 0.238708 \\
\hline 103 & I & 3.304335 & 0.414136 & 3.044789 \\
\hline
\end{tabular}


Vinyl Cation Intermediate 16a'

\begin{tabular}{|c|c|c|c|c|c|}
\hline & No. & Atom & $X$ & Y & Z \\
\hline & 23 & $\mathrm{C}$ & 4.020117 & 2.266761 & -0.302052 \\
\hline & 24 & $\mathrm{C}$ & 4.275864 & 3.638999 & -0.383353 \\
\hline & 25 & $\mathrm{H}$ & 3.418532 & 5.619811 & -0.398076 \\
\hline & & & 4.841434 & 1.557409 & -0.339358 \\
\hline & & & 2.514835 & 0.739949 & -0.106005 \\
\hline & & & 1.086559 & 4.808607 & -0.168888 \\
\hline & & & 5.296950 & 3.994940 & -0.484029 \\
\hline & & & -0.929067 & -0.982649 & 0.145360 \\
\hline & & & 0.276635 & -1.448288 & 0.065466 \\
\hline & & & 1.231916 & -1.666989 & 1.163782 \\
\hline & & & 1.207775 & -0.791169 & 2.266867 \\
\hline & & C & 2.121876 & -2.753820 & 1.157763 \\
\hline & 35 & $\mathrm{C}$ & 2.054117 & -1.014418 & 3.350472 \\
\hline$z$ & 36 & $\mathrm{H}$ & 0.544354 & 0.067662 & 2.255428 \\
\hline 0.293858 & 37 & $\mathrm{C}$ & 2.962616 & -2.967745 & 2.246571 \\
\hline 0.224859 & 38 & $\mathrm{H}$ & 2.152313 & -3.427195 & 0.307461 \\
\hline 0.255287 & 39 & $\mathrm{C}$ & 2.930075 & -2.102216 & 3.344416 \\
\hline 0.352618 & 40 & $\mathrm{H}$ & 2.035995 & -0.331500 & 4.194421 \\
\hline 0.418127 & 41 & $\mathrm{H}$ & 3.647405 & -3.810188 & 2.236483 \\
\hline 0.392108 & 42 & $\mathrm{H}$ & 3.592955 & -2.270878 & 4.187771 \\
\hline 0.265197 & 43 & I & 0.866940 & -1.821216 & -2.006048 \\
\hline 0.198049 & & & & & \\
\hline 0.386182 & & & & & \\
\hline
\end{tabular}

SCF Done: $\quad E($ RB3LYP $)=-1011.43146181$

\begin{tabular}{|c|c|c|c|c|}
\hline No. & Atom & $\mathrm{X}$ & $\mathrm{Y}$ & $\mathrm{Z}$ \\
\hline 1 & $\mathrm{C}$ & -3.054267 & -1.991636 & 0.293858 \\
\hline 2 & $\mathrm{C}$ & -2.276247 & -0.788539 & 0.224859 \\
\hline 3 & $\mathrm{C}$ & -2.943749 & 0.507680 & 0.255287 \\
\hline 4 & $\mathrm{C}$ & -4.375803 & 0.502431 & 0.352618 \\
\hline 5 & $\mathrm{C}$ & -5.077384 & -0.722620 & 0.418127 \\
\hline 6 & $\mathrm{C}$ & -4.433341 & -1.959938 & 0.392108 \\
\hline 7 & $\mathrm{H}$ & -2.525356 & -2.938661 & 0.265197 \\
\hline 8 & $\mathrm{C}$ & -2.270975 & 1.758829 & 0.198049 \\
\hline 9 & $\mathrm{C}$ & -5.106529 & 1.718369 & 0.386182 \\
\hline 10 & $\mathrm{H}$ & -6.161630 & -0.687991 & 0.491280 \\
\hline 11 & $\mathrm{H}$ & -5.004646 & -2.880413 & 0.443937 \\
\hline 12 & $\mathrm{C}$ & -4.439723 & 2.925534 & 0.328063 \\
\hline 13 & $\mathrm{C}$ & -3.043273 & 2.939285 & 0.236959 \\
\hline 14 & $\mathrm{H}$ & -6.189338 & 1.681407 & 0.458049 \\
\hline 15 & $\mathrm{H}$ & -4.986576 & 3.862378 & 0.352728 \\
\hline 16 & $\mathrm{H}$ & -2.518010 & 3.887527 & 0.192160 \\
\hline 17 & $\mathrm{C}$ & -0.864963 & 1.916957 & 0.100181 \\
\hline 18 & $\mathrm{C}$ & 0.297582 & 2.277152 & 0.001352 \\
\hline 19 & $\mathrm{C}$ & 1.642496 & 2.718974 & -0.124115 \\
\hline 20 & $\mathrm{C}$ & 1.910691 & 4.103246 & -0.205642 \\
\hline 21 & $\mathrm{C}$ & 2.714875 & 1.803482 & -0.173101 \\
\hline 22 & $\mathrm{C}$ & 3.219755 & 4.554261 & -0.334748 \\
\hline
\end{tabular}




\section{Vinyl Cation Intermediate 16b'}

\begin{tabular}{|c|c|c|c|c|}
\hline No. & Atom & $\mathrm{X}$ & Y & Z \\
\hline 32 & $\mathrm{C}$ & 0.854526 & -3.477614 & -2.124323 \\
\hline 33 & $\mathrm{C}$ & 3.178271 & -3.464040 & -1.508941 \\
\hline 34 & $\mathrm{C}$ & 2.141233 & -4.012896 & -2.272224 \\
\hline & $\mathrm{I}$ & 0.033964 & -3.901953 & -2.697691 \\
\hline & $\mathrm{I}$ & 4.179586 & -3.877385 & -1.600185 \\
\hline & [ & 0.759980 & -1.238573 & 2.517714 \\
\hline & $z$ & 4.132773 & -1.858167 & 0.165939 \\
\hline & $\mathrm{I}$ & 4.492007 & -0.908745 & -0.252998 \\
\hline & $\mathrm{I}$ & 3.871272 & -1.684079 & 1.215899 \\
\hline & $\mathrm{I}$ & 4.974168 & -2.555826 & 0.144181 \\
\hline & z & 2.398828 & -5.141833 & -3.237471 \\
\hline & $\mathrm{I}$ & 2.456800 & -4.766719 & -4.267784 \\
\hline & $\mathrm{I}$ & 3.341346 & -5.652084 & -3.018919 \\
\hline$y$ & $\mathrm{I}$ & 1.592137 & -5.881991 & -3.209448 \\
\hline 40 & ¿ & -0.817978 & -1.897955 & -1.108050 \\
\hline $47 \mathrm{c}$ & esH & -1.195725 & -2.042317 & -0.088286 \\
\hline 48 & $\mathrm{H}$ & -0.869358 & -0.824358 & -1.312587 \\
\hline 49 & $\mathrm{H}$ & -1.499617 & -2.411607 & -1.790909 \\
\hline 50 & $\mathrm{C}$ & -3.420316 & 0.693675 & -2.782044 \\
\hline
\end{tabular}

SCF Done: $\quad E(R B 3 L Y P)=-1247.33120316 \quad$ A.U. after

\begin{tabular}{|c|c|c|c|c|}
\hline No. & Atom & $\mathrm{X}$ & $\mathrm{Y}$ & $\mathrm{Z}$ \\
\hline 1 & $\mathrm{C}$ & 3.461213 & 1.833679 & 0.552705 \\
\hline 2 & $\mathrm{C}$ & 2.089515 & 1.751401 & 0.145588 \\
\hline 3 & $\mathrm{C}$ & 1.427952 & 2.933223 & -0.398670 \\
\hline 4 & $\mathrm{C}$ & 2.246248 & 4.100574 & -0.592813 \\
\hline 5 & $\mathrm{C}$ & 3.599301 & 4.100519 & -0.184623 \\
\hline 6 & $\mathrm{C}$ & 4.203023 & 2.992115 & 0.403515 \\
\hline 7 & $\mathrm{H}$ & 3.914312 & 0.947339 & 0.981374 \\
\hline 8 & $\mathrm{C}$ & 0.053946 & 3.009549 & -0.753846 \\
\hline 9 & $\mathrm{C}$ & 1.712891 & 5.275060 & -1.182873 \\
\hline 10 & $\mathrm{H}$ & 4.179048 & 5.007688 & -0.336204 \\
\hline 11 & $\mathrm{H}$ & 5.239322 & 3.028898 & 0.721474 \\
\hline 12 & $\mathrm{C}$ & 0.390741 & 5.312453 & -1.572832 \\
\hline 13 & $\mathrm{C}$ & -0.422449 & 4.195271 & -1.349570 \\
\hline 14 & $\mathrm{H}$ & 2.360349 & 6.136143 & -1.318578 \\
\hline 15 & $\mathrm{H}$ & -0.028786 & 6.202232 & -2.030872 \\
\hline 16 & $\mathrm{H}$ & -1.471578 & 4.232805 & -1.622771 \\
\hline 17 & $\mathrm{C}$ & -0.912145 & 1.990619 & -0.515620 \\
\hline 18 & $\mathrm{C}$ & -1.978903 & 1.408486 & -0.375854 \\
\hline 19 & $\mathrm{C}$ & -3.212411 & 0.713229 & -0.238873 \\
\hline
\end{tabular}




\begin{tabular}{|l|l|l|l|l|}
\hline 20 & $\mathrm{C}$ & -3.725008 & 0.415631 & 1.050412 \\
\hline 21 & $\mathrm{C}$ & -3.937658 & 0.350940 & -1.406114 \\
\hline 22 & $\mathrm{C}$ & -4.939419 & -0.263431 & 1.141926 \\
\hline 23 & $\mathrm{C}$ & -5.145300 & -0.327030 & -1.254144 \\
\hline 24 & $\mathrm{C}$ & -5.665766 & -0.645190 & 0.007479 \\
\hline 25 & $\mathrm{H}$ & -5.335672 & -0.495581 & 2.127637 \\
\hline 26 & $\mathrm{H}$ & -5.700918 & -0.612895 & -2.144269 \\
\hline 27 & $\mathrm{C}$ & 1.564832 & 0.490273 & 0.256679 \\
\hline 28 & $\mathrm{C}$ & 1.412311 & -0.776722 & 0.441913 \\
\hline 29 & $\mathrm{C}$ & 1.658658 & -1.877858 & -0.506646 \\
\hline 30 & $\mathrm{C}$ & 0.585258 & -2.422505 & -1.255168 \\
\hline 31 & $\mathrm{C}$ & 2.969566 & -2.403632 & -0.627076 \\
\hline
\end{tabular}

\begin{tabular}{|l|l|l|l|l|}
\hline 51 & $\mathrm{H}$ & -2.442389 & 0.236820 & -2.976339 \\
\hline 52 & $\mathrm{H}$ & -4.112075 & 0.349297 & -3.555596 \\
\hline 53 & $\mathrm{H}$ & -3.293748 & 1.776583 & -2.903000 \\
\hline 54 & $\mathrm{C}$ & -6.993027 & -1.350528 & 0.138604 \\
\hline 55 & $\mathrm{H}$ & -7.820697 & -0.629004 & 0.122930 \\
\hline 56 & $\mathrm{H}$ & -7.159046 & -2.050785 & -0.686519 \\
\hline 57 & $\mathrm{H}$ & -7.062296 & -1.905365 & 1.079393 \\
\hline 58 & $\mathrm{C}$ & -2.995536 & 0.853458 & 2.295506 \\
\hline 59 & $\mathrm{H}$ & -1.952265 & 0.520370 & 2.293134 \\
\hline 60 & $\mathrm{H}$ & -2.981644 & 1.947406 & 2.381081 \\
\hline 61 & $\mathrm{H}$ & -3.478464 & 0.454975 & 3.192064 \\
\hline & & & & \\
\hline
\end{tabular}




\section{Bridged Radical Intermediate 17a}

\begin{tabular}{|c|c|c|c|c|}
\hline No. & Atom & $\mathrm{X}$ & $\mathrm{Y}$ & $\mathrm{Z}$ \\
\hline 23 & $\mathrm{C}$ & 3.702214 & 2.105032 & -1.381893 \\
\hline 24 & $\mathrm{C}$ & 4.215815 & 3.346135 & -0.995544 \\
\hline 25 & $\mathrm{H}$ & 3.797788 & 5.218169 & -0.005765 \\
\hline 26 & $\mathrm{H}$ & 4.328893 & 1.398888 & -1.919557 \\
\hline 27 & $\mathrm{H}$ & 1.983176 & 0.800499 & -1.387234 \\
\hline 28 & $\mathrm{H}$ & 1.449815 & 4.621016 & 0.529119 \\
\hline 29 & $\mathrm{H}$ & 5.243659 & 3.608119 & -1.231744 \\
\hline 30 & $\mathrm{C}$ & -0.885695 & -0.831896 & 0.818544 \\
\hline 31 & $\mathrm{C}$ & 0.271726 & -1.122317 & 1.108469 \\
\hline 32 & $\mathrm{C}$ & 1.587011 & -1.344098 & 1.601764 \\
\hline 33 & $\mathrm{C}$ & 2.382842 & -0.252243 & 2.007587 \\
\hline 34 & $\mathrm{C}$ & 2.097071 & -2.653607 & 1.723046 \\
\hline $35 \mathrm{cycle} \mathrm{C}$ & 3.655933 & -0.472390 & 2.525524 \\
\hline 36 & $\mathrm{H}$ & 1.990984 & 0.754044 & 1.909701 \\
\hline 37 & $\mathrm{C}$ & 3.371477 & -2.860531 & 2.239437 \\
\hline 38 & $\mathrm{H}$ & 1.489886 & -3.491224 & 1.395967 \\
\hline 39 & $\mathrm{C}$ & 4.153468 & -1.773022 & 2.642714 \\
\hline 40 & $\mathrm{H}$ & 4.262727 & 0.373933 & 2.834612 \\
\hline 41 & $\mathrm{H}$ & 3.759262 & -3.871671 & 2.325083 \\
\hline 42 & $\mathrm{H}$ & 5.149199 & -1.939922 & 3.044340 \\
\hline 43 & $\mathrm{I}$ & 0.358542 & -2.055321 & -1.999978 \\
\hline & & & & \\
\hline
\end{tabular}

SCF Done: $\quad E(U B 3 L Y P)=-1011.66200888 \quad$ A.U. after

\begin{tabular}{|c|c|c|c|c|}
\hline No. & Atom & $X$ & Y & Z \\
\hline 1 & $\mathrm{C}$ & -3.019746 & -1.895235 & 0.776068 \\
\hline 2 & $\mathrm{C}$ & -2.291318 & -0.704864 & 0.672639 \\
\hline 3 & $\mathrm{C}$ & -2.983845 & 0.549959 & 0.511586 \\
\hline 4 & $\mathrm{C}$ & -4.420816 & 0.520565 & 0.540192 \\
\hline 5 & $\mathrm{C}$ & -5.108007 & -0.716511 & 0.655738 \\
\hline 6 & $\mathrm{C}$ & -4.421931 & -1.906317 & 0.755625 \\
\hline 7 & $\mathrm{H}$ & -2.470866 & -2.824848 & 0.881445 \\
\hline 8 & $\mathrm{C}$ & -2.341965 & 1.824981 & 0.332497 \\
\hline 9 & $\mathrm{C}$ & -5.164181 & 1.726536 & 0.453663 \\
\hline 10 & $\mathrm{H}$ & -6.194907 & -0.704032 & 0.661775 \\
\hline 11 & $\mathrm{H}$ & -4.954800 & -2.849053 & 0.834695 \\
\hline 12 & $\mathrm{C}$ & -4.525099 & 2.939033 & 0.334514 \\
\hline 13 & $\mathrm{C}$ & -3.122899 & 2.981826 & 0.265809 \\
\hline 14 & $\mathrm{H}$ & -6.249095 & 1.670575 & 0.484592 \\
\hline 15 & $\mathrm{H}$ & -5.094651 & 3.861826 & 0.273928 \\
\hline 16 & $\mathrm{H}$ & -2.617032 & 3.932625 & 0.133728 \\
\hline 17 & $\mathrm{C}$ & -0.938825 & 1.999837 & 0.154586 \\
\hline 18 & $\mathrm{C}$ & 0.207183 & 2.331747 & -0.087532 \\
\hline 19 & $\mathrm{C}$ & 1.559087 & 2.669340 & -0.388226 \\
\hline 20 & $\mathrm{C}$ & 2.085197 & 3.920252 & -0.004187 \\
\hline 21 & $\mathrm{C}$ & 2.385697 & 1.762104 & -1.083691 \\
\hline 22 & $\mathrm{C}$ & 3.403476 & 4.251196 & -0.306574 \\
\hline
\end{tabular}




\section{Bridged Radical Intermediate 17b}

\begin{tabular}{|c|c|c|c|c|}
\hline No. & Atom & $X$ & Y & Z \\
\hline 32 & $\mathrm{C}$ & -0.602740 & 3.767590 & -0.992644 \\
\hline 33 & $\mathrm{C}$ & 2.138460 & 3.524488 & -1.532768 \\
\hline 34 & $\mathrm{C}$ & 0.176609 & 4.888620 & -1.263061 \\
\hline & $\mathrm{C}$ & 1.548393 & 4.793208 & -1.533286 \\
\hline & $\mathrm{H}$ & 3.200910 & 3.432350 & -1.747399 \\
\hline & $\mathrm{H}$ & -0.297953 & 5.867461 & -1.265758 \\
\hline & $\mathrm{C}$ & 2.836589 & -2.768632 & -2.434248 \\
\hline 48 & $\mathrm{H}$ & 2.205645 & -3.665729 & -2.425194 \\
\hline & $\mathrm{H}$ & 2.221255 & -1.959776 & -2.846533 \\
\hline & $\mathrm{H}$ & 3.670208 & -2.945899 & -3.120716 \\
\hline & $\mathrm{C}$ & 6.689466 & -2.045295 & 0.751525 \\
\hline & $\mathrm{H}$ & 7.066533 & -3.045199 & 1.006361 \\
\hline & $\mathrm{H}$ & 7.247339 & -1.704636 & -0.127676 \\
\hline 45 & $\mathrm{H}$ & 6.935740 & -1.382938 & 1.588159 \\
\hline $46 \mathrm{c}$ & ${ }_{\text {les }}^{C}$ & 1.944231 & -1.633684 & 2.442529 \\
\hline 47 & $\mathrm{H}$ & 1.270469 & -0.794002 & 2.237287 \\
\hline 48 & $\mathrm{H}$ & 1.304360 & -2.507672 & 2.615363 \\
\hline 49 & $\mathrm{H}$ & 2.482534 & -1.417899 & 3.370414 \\
\hline 50 & $\mathrm{C}$ & 2.087117 & 1.027091 & -1.233263 \\
\hline 51 & $\mathrm{H}$ & 2.217556 & 0.677353 & -0.202682 \\
\hline
\end{tabular}

SCF Done: $\quad E(U B 3 L Y P)=-1247.56318824 \quad$ A.U. after

\begin{tabular}{|c|c|c|c|c|}
\hline No. & Atom & $\mathrm{X}$ & Y & Z \\
\hline 1 & $\mathrm{C}$ & -3.897872 & 0.222296 & -0.722828 \\
\hline 2 & $\mathrm{C}$ & -2.680574 & -0.470518 & -0.691386 \\
\hline 3 & $\mathrm{C}$ & -2.679337 & -1.911320 & -0.765487 \\
\hline 4 & $\mathrm{C}$ & -3.953887 & -2.564839 & -0.920397 \\
\hline 5 & $\mathrm{C}$ & -5.153448 & -1.807169 & -0.951257 \\
\hline 6 & $\mathrm{C}$ & -5.129171 & -0.435668 & -0.843654 \\
\hline 7 & $\mathrm{H}$ & -3.871815 & 1.303632 & -0.649899 \\
\hline 8 & $\mathrm{C}$ & -1.509935 & -2.744254 & -0.702219 \\
\hline 9 & $\mathrm{C}$ & -4.030609 & -3.976084 & -1.044894 \\
\hline 10 & $\mathrm{H}$ & -6.095857 & -2.337633 & -1.060492 \\
\hline 11 & $\mathrm{H}$ & -6.049243 & 0.140784 & -0.860319 \\
\hline 12 & $\mathrm{C}$ & -2.892815 & -4.747064 & -1.015170 \\
\hline 13 & $\mathrm{C}$ & -1.644781 & -4.128899 & -0.838955 \\
\hline 14 & $\mathrm{H}$ & -5.009600 & -4.432734 & -1.164269 \\
\hline 15 & $\mathrm{H}$ & -2.949935 & -5.827275 & -1.113091 \\
\hline 16 & $\mathrm{H}$ & -0.745902 & -4.734217 & -0.788647 \\
\hline 17 & $\mathrm{C}$ & -0.174435 & -2.286848 & -0.472551 \\
\hline 18 & $\mathrm{C}$ & 1.022944 & -2.235335 & -0.246587 \\
\hline 19 & $\mathrm{C}$ & 2.426311 & -2.175576 & 0.005182 \\
\hline 20 & $\mathrm{C}$ & 3.337440 & -2.434589 & -1.050082 \\
\hline
\end{tabular}




\begin{tabular}{|l|l|l|l|l|}
\hline 21 & $\mathrm{C}$ & 2.904772 & -1.879515 & 1.306167 \\
\hline 22 & $\mathrm{C}$ & 4.705978 & -2.373603 & -0.786807 \\
\hline 23 & $\mathrm{C}$ & 4.284718 & -1.830747 & 1.515083 \\
\hline 24 & $\mathrm{C}$ & 5.202297 & -2.073623 & 0.487375 \\
\hline 25 & $\mathrm{H}$ & 5.405092 & -2.566590 & -1.598286 \\
\hline 26 & $\mathrm{H}$ & 4.653440 & -1.598570 & 2.512127 \\
\hline 27 & $\mathrm{C}$ & -1.535602 & 0.373816 & -0.635540 \\
\hline 28 & $\mathrm{I}$ & -1.283050 & 1.281794 & 2.538060 \\
\hline 29 & $\mathrm{C}$ & -0.779444 & 1.340080 & -0.743456 \\
\hline 30 & $\mathrm{C}$ & 0.019883 & 2.485920 & -0.989857 \\
\hline 31 & $\mathrm{C}$ & 1.408535 & 2.368430 & -1.260762 \\
\hline
\end{tabular}

\begin{tabular}{|l|l|l|l|l|}
\hline 52 & $\mathrm{H}$ & 1.495696 & 0.263390 & -1.746971 \\
\hline 53 & $\mathrm{H}$ & 3.077876 & 1.074671 & -1.694920 \\
\hline 54 & $\mathrm{C}$ & 2.370278 & 6.031812 & -1.793181 \\
\hline 55 & $\mathrm{H}$ & 1.789757 & 6.795197 & -2.322538 \\
\hline 56 & $\mathrm{H}$ & 2.711260 & 6.480786 & -0.850359 \\
\hline 57 & $\mathrm{H}$ & 3.261648 & 5.805813 & -2.387442 \\
\hline 58 & $\mathrm{C}$ & -2.075121 & 3.915292 & -0.706611 \\
\hline 59 & $\mathrm{H}$ & -2.680822 & 3.374232 & -1.444066 \\
\hline 60 & $\mathrm{H}$ & -2.326567 & 3.510203 & 0.281303 \\
\hline 61 & $\mathrm{H}$ & -2.373472 & 4.967549 & -0.732424 \\
\hline & & & & \\
\hline
\end{tabular}




\section{Bridged Radical Intermediate 17c}

SCF Done: $\quad E(U B 3 L Y P)=-2097.86588200$

A.U. after

\begin{tabular}{|c|c|c|c|c|}
\hline No. & Atom & $\mathrm{X}$ & $\mathrm{Y}$ & $\mathrm{Z}$ \\
\hline 1 & $\mathrm{C}$ & -7.758731 & 2.591598 & -0.817719 \\
\hline 2 & $\mathrm{C}$ & -8.396123 & 3.180880 & 0.280271 \\
\hline 3 & $\mathrm{C}$ & -6.439765 & 2.141795 & -0.755737 \\
\hline 4 & $\mathrm{C}$ & -7.674584 & 3.313343 & 1.472060 \\
\hline 5 & $\mathrm{C}$ & -5.729150 & 2.283197 & 0.463220 \\
\hline 6 & $\mathrm{C}$ & -6.353345 & 2.880774 & 1.587401 \\
\hline 7 & $\mathrm{C}$ & 6.025931 & 3.940326 & -1.562272 \\
\hline 8 & $\mathrm{C}$ & -4.379319 & 1.836060 & 0.558687 \\
\hline 9 & $\mathrm{C}$ & 4.837881 & 3.292217 & -1.225780 \\
\hline 10 & $\mathrm{C}$ & -1.112435 & 2.287485 & 1.292287 \\
\hline 11 & $\mathrm{C}$ & 7.119036 & 3.992154 & -0.689827 \\
\hline 12 & $\mathrm{C}$ & -3.234767 & 1.419265 & 0.623525 \\
\hline 13 & $\mathrm{C}$ & 0.279268 & 2.306528 & 1.289623 \\
\hline 14 & $\mathrm{C}$ & 4.739441 & 2.661514 & 0.043352 \\
\hline 15 & $\mathrm{C}$ & -1.827824 & 1.224931 & 0.737075 \\
\hline 16 & $\mathrm{C}$ & 3.510239 & 2.018671 & 0.390520 \\
\hline 17 & $\mathrm{C}$ & 6.992926 & 3.380902 & 0.560635 \\
\hline 18 & $\mathrm{C}$ & -7.557771 & -3.259999 & -1.282205 \\
\hline 19 & $\mathrm{C}$ & 5.827512 & 2.715328 & 0.946524 \\
\hline
\end{tabular}

\begin{tabular}{|c|c|c|c|c|}
\hline No. & Atom & $\mathrm{X}$ & $\mathrm{Y}$ & $\mathrm{Z}$ \\
\hline 53 & $\mathrm{H}$ & 0.900065 & -3.010399 & -1.093038 \\
\hline 54 & $\mathrm{H}$ & 6.208780 & -1.887834 & 4.259502 \\
\hline 55 & $\mathrm{C}$ & 3.678774 & 3.273394 & -2.190068 \\
\hline 6 & $\mathrm{H}$ & 3.911737 & 3.851354 & -3.089721 \\
\hline 7 & $\mathrm{H}$ & 3.435242 & 2.249022 & -2.494201 \\
\hline 8 & $\mathrm{H}$ & 2.774711 & 3.696429 & -1.735552 \\
\hline 9 & $\mathrm{C}$ & 5.749248 & 2.061573 & 2.303236 \\
\hline 0 & $\mathrm{H}$ & 4.793635 & 2.268401 & 2.797096 \\
\hline 1 & $\mathrm{H}$ & 5.841231 & 0.971358 & 2.223072 \\
\hline 2 & $\mathrm{H}$ & 6.555766 & 2.414891 & 2.953846 \\
\hline 3 & $\mathrm{C}$ & 5.161595 & -3.857826 & -0.657155 \\
\hline 4 & $\mathrm{H}$ & 4.128942 & -4.219616 & -0.720798 \\
\hline 5 & $\mathrm{H}$ & 5.273212 & -3.106941 & -1.449466 \\
\hline 6 & $\mathrm{H}$ & 5.834090 & -4.691843 & -0.879015 \\
\hline 7 & $\mathrm{C}$ & 3.893072 & -0.891285 & 3.287476 \\
\hline 68 & $\mathrm{H}$ & 4.320676 & -0.497627 & 4.214517 \\
\hline 39 & $\mathrm{H}$ & 3.599833 & -0.050866 & 2.652671 \\
\hline 70 & $\mathrm{H}$ & 2.972524 & -1.430842 & 3.546407 \\
\hline 71 & $\mathrm{C}$ & -5.603225 & 3.039943 & 2.887252 \\
\hline
\end{tabular}




\begin{tabular}{|c|c|c|c|c|}
\hline 20 & $\mathrm{C}$ & 2.419127 & 1.503115 & 0.575615 \\
\hline 21 & $\mathrm{C}$ & 1.022347 & 1.252568 & 0.755799 \\
\hline 22 & $\mathrm{C}$ & -6.236645 & -2.815336 & -1.330466 \\
\hline 23 & $\mathrm{C}$ & -8.347106 & -3.115675 & -0.135421 \\
\hline 24 & $\mathrm{C}$ & -1.111613 & 0.070692 & 0.260935 \\
\hline 25 & $\mathrm{C}$ & 0.333227 & 0.075465 & 0.301082 \\
\hline 26 & $\mathrm{C}$ & -5.683967 & -2.191470 & -0.182552 \\
\hline 27 & $\mathrm{C}$ & -3.195945 & -1.297457 & -0.218394 \\
\hline 28 & $\mathrm{C}$ & -7.779318 & -2.501711 & 0.987187 \\
\hline 29 & $\mathrm{C}$ & -4.337658 & -1.729303 & -0.210231 \\
\hline 30 & $\mathrm{C}$ & -1.790302 & -1.087768 & -0.263902 \\
\hline 31 & $\mathrm{C}$ & -6.464170 & -2.037848 & 0.992216 \\
\hline 32 & $\mathrm{C}$ & 1.043687 & -1.104031 & -0.127042 \\
\hline 33 & $\mathrm{C}$ & 2.408471 & -1.346172 & 0.168375 \\
\hline 34 & $\mathrm{C}$ & 5.457815 & -3.276255 & 0.697991 \\
\hline 35 & $\mathrm{C}$ & -1.041757 & -2.136670 & -0.809095 \\
\hline 36 & $\mathrm{C}$ & 0.344819 & -2.151117 & -0.733399 \\
\hline 37 & $\mathrm{C}$ & 6.585627 & -3.685194 & 1.409496 \\
\hline 38 & $\mathrm{C}$ & 3.453300 & -1.827374 & 0.602342 \\
\hline 39 & $\mathrm{C}$ & 4.604773 & -2.298653 & 1.280125 \\
\hline 40 & $\mathrm{C}$ & 6.890467 & -3.180237 & 2.678149 \\
\hline 41 & $\mathrm{C}$ & 4.868257 & -1.809361 & 2.593118 \\
\hline 42 & $\mathrm{C}$ & 6.008070 & -2.257187 & 3.256078 \\
\hline 43 & $\mathrm{H}$ & -8.305760 & 2.480005 & -1.751785 \\
\hline 44 & $\mathrm{H}$ & -8.155386 & 3.765990 & 2.337053 \\
\hline 45 & $\mathrm{H}$ & 6.098669 & 4.422867 & -2.534880 \\
\hline 46 & $\mathrm{H}$ & -1.662448 & 3.144601 & 1.666045 \\
\hline 47 & $\mathrm{H}$ & 0.806925 & 3.183778 & 1.648842 \\
\hline 48 & $\mathrm{H}$ & -7.984584 & -3.731542 & -2.165140 \\
\hline 49 & $\mathrm{H}$ & 7.825895 & 3.423062 & 1.259712 \\
\hline 50 & $\mathrm{H}$ & -8.379727 & -2.381028 & 1.886606 \\
\hline 51 & $\mathrm{H}$ & 7.244302 & -4.425712 & 0.961050 \\
\hline 52 & $\mathrm{H}$ & -1.568758 & -2.985249 & -1.231560 \\
\hline
\end{tabular}

\begin{tabular}{|c|c|c|c|c|}
\hline 72 & $\mathrm{H}$ & -5.283426 & 2.070585 & 3.288738 \\
\hline 73 & $\mathrm{H}$ & -4.693613 & 3.638005 & 2.752461 \\
\hline 74 & $\mathrm{H}$ & -6.224659 & 3.532087 & 3.641631 \\
\hline 75 & $\mathrm{C}$ & -5.775748 & 1.532715 & -1.965583 \\
\hline 76 & $\mathrm{H}$ & -5.374275 & 0.538296 & -1.742972 \\
\hline 77 & $\mathrm{H}$ & -6.482050 & 1.444262 & -2.796727 \\
\hline 78 & $\mathrm{H}$ & -4.929373 & 2.144496 & -2.302228 \\
\hline 79 & $\mathrm{C}$ & -5.875061 & -1.404677 & 2.228066 \\
\hline 80 & $\mathrm{H}$ & -5.452106 & -0.418611 & 2.009087 \\
\hline 81 & $\mathrm{H}$ & -6.632878 & -1.292904 & 3.009490 \\
\hline 82 & $\mathrm{H}$ & -5.057785 & -2.014975 & 2.632919 \\
\hline 83 & $\mathrm{C}$ & -5.413916 & -2.985334 & -2.584022 \\
\hline 84 & $\mathrm{H}$ & -5.088881 & -2.018182 & -2.986404 \\
\hline 85 & $\mathrm{H}$ & -4.503463 & -3.565121 & -2.388913 \\
\hline 86 & $\mathrm{H}$ & -5.985279 & -3.501722 & -3.361284 \\
\hline 87 & $\mathrm{C}$ & 8.140249 & -3.613288 & 3.404577 \\
\hline 88 & $\mathrm{H}$ & 8.980723 & -2.945364 & 3.171258 \\
\hline 89 & $\mathrm{H}$ & 8.001956 & -3.592369 & 4.490864 \\
\hline 90 & $\mathrm{H}$ & 8.441795 & -4.625964 & 3.116848 \\
\hline 91 & $\mathrm{C}$ & 8.402780 & 4.675603 & -1.097610 \\
\hline 92 & $\mathrm{H}$ & 9.027149 & 4.011815 & -1.710924 \\
\hline 93 & $\mathrm{H}$ & 8.207466 & 5.574404 & -1.693330 \\
\hline 94 & $\mathrm{H}$ & 8.996694 & 4.968448 & -0.225358 \\
\hline 95 & $\mathrm{C}$ & -9.764705 & -3.635433 & -0.099437 \\
\hline 96 & $\mathrm{H}$ & -9.798154 & -4.670479 & 0.267351 \\
\hline 97 & $\mathrm{H}$ & -10.394939 & -3.035959 & 0.566304 \\
\hline 98 & $\mathrm{H}$ & -10.219906 & -3.629895 & -1.095594 \\
\hline 99 & $\mathrm{C}$ & -9.815076 & 3.687423 & 0.172413 \\
\hline 100 & $\mathrm{H}$ & -9.839238 & 4.729135 & -0.175758 \\
\hline 101 & $\mathrm{H}$ & -10.399488 & 3.094987 & -0.539847 \\
\hline 102 & $\mathrm{H}$ & -10.326735 & 3.657482 & 1.140414 \\
\hline 103 & I & 3.810819 & -0.950972 & -2.928718 \\
\hline
\end{tabular}




\section{Iodovinyl Cation 18}

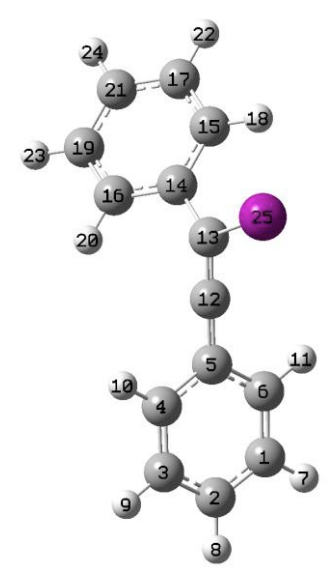

SCF Done: $\quad E($ RB3LYP $)=-550.687703113$

A.U. after

\begin{tabular}{|c|c|c|c|c|}
\hline No. & Atom & $\mathrm{X}$ & $\mathrm{Y}$ & $\mathrm{Z}$ \\
\hline 14 & $\mathrm{C}$ & -1.586691 & 0.940518 & 0.011621 \\
\hline 15 & $\mathrm{C}$ & -2.699562 & 0.696105 & 0.827648 \\
\hline 16 & $\mathrm{C}$ & -1.528170 & 2.106151 & -0.769737 \\
\hline 17 & $\mathrm{C}$ & -3.738117 & 1.616884 & 0.865351 \\
\hline 18 & $\mathrm{H}$ & -2.751891 & -0.206754 & 1.424902 \\
\hline 19 & $\mathrm{C}$ & -2.575362 & 3.018588 & -0.725849 \\
\hline 20 & $\mathrm{H}$ & -0.680479 & 2.280979 & -1.422825 \\
\hline 21 & $\mathrm{C}$ & -3.678169 & 2.777234 & 0.091477 \\
\hline 22 & $\mathrm{H}$ & -4.600017 & 1.426431 & 1.494561 \\
\hline 23 & $\mathrm{H}$ & -2.534755 & 3.912437 & -1.337878 \\
\hline 24 & $\mathrm{H}$ & -4.496706 & 3.487711 & 0.119064 \\
\hline 25 & $\mathrm{I}$ & -0.734298 & -2.144469 & -0.133663 \\
\hline & & & & \\
\hline 6
\end{tabular}

\begin{tabular}{|c|c|c|c|c|}
\hline No. & Atom & $\mathrm{X}$ & $\mathrm{Y}$ & $\mathrm{Z}$ \\
\hline 1 & $\mathrm{C}$ & 4.164864 & 0.878557 & 1.369442 \\
\hline 2 & $\mathrm{C}$ & 4.863651 & 1.115053 & 0.175908 \\
\hline 3 & $\mathrm{C}$ & 4.227539 & 1.060242 & -1.073820 \\
\hline 4 & $\mathrm{C}$ & 2.882006 & 0.771648 & -1.142167 \\
\hline 5 & $\mathrm{C}$ & 2.149234 & 0.526247 & 0.062197 \\
\hline 6 & $\mathrm{C}$ & 2.820284 & 0.580567 & 1.324967 \\
\hline 7 & $\mathrm{H}$ & 4.683378 & 0.927988 & 2.319424 \\
\hline 8 & $\mathrm{H}$ & 5.923023 & 1.345047 & 0.220069 \\
\hline 9 & $\mathrm{H}$ & 4.794352 & 1.245301 & -1.978406 \\
\hline 10 & $\mathrm{H}$ & 2.366602 & 0.721151 & -2.093930 \\
\hline 11 & $\mathrm{H}$ & 2.256556 & 0.390480 & 2.230445 \\
\hline 12 & $\mathrm{C}$ & 0.809541 & 0.255964 & 0.012082 \\
\hline 13 & $\mathrm{C}$ & -0.451427 & 0.010587 & -0.011003 \\
\hline
\end{tabular}

6 cycles 


\section{Bridged Iodonium Ion 18'}

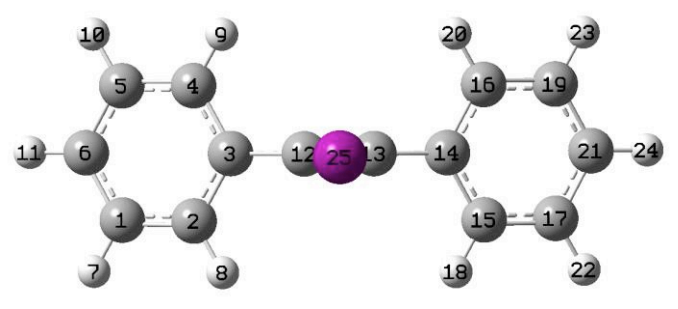

SCF Done: $\quad E(R B 3 L Y P)=-550.563465340$

A.U. after

\begin{tabular}{|c|c|c|c|c|}
\hline No. & Atom & $\mathrm{X}$ & $\mathrm{Y}$ & $\mathrm{Z}$ \\
\hline 1 & $\mathrm{C}$ & -1.220105 & 3.938844 & -1.514466 \\
\hline 2 & $\mathrm{C}$ & -1.230695 & 2.636655 & -1.038340 \\
\hline 3 & $\mathrm{C}$ & 0.000000 & 1.971081 & -0.808103 \\
\hline 4 & $\mathrm{C}$ & 1.230695 & 2.636655 & -1.038340 \\
\hline 5 & $\mathrm{C}$ & 1.220105 & 3.938844 & -1.514466 \\
\hline 6 & $\mathrm{C}$ & 0.000000 & 4.587831 & -1.751056 \\
\hline 7 & $\mathrm{H}$ & -2.156285 & 4.454758 & -1.701040 \\
\hline 8 & $\mathrm{H}$ & -2.165662 & 2.121272 & -0.844995 \\
\hline 9 & $\mathrm{H}$ & 2.165662 & 2.121272 & -0.844995 \\
\hline 10 & $\mathrm{H}$ & 2.156285 & 4.454758 & -1.701040 \\
\hline 11 & $\mathrm{H}$ & 0.000000 & 5.609035 & -2.120484 \\
\hline 12 & $\mathrm{C}$ & 0.000000 & 0.632932 & -0.367353 \\
\hline 13 & $\mathrm{C}$ & 0.000000 & -0.632932 & -0.367353 \\
\hline
\end{tabular}

\begin{tabular}{|c|c|c|c|c|}
\hline No. & Atom & $\mathrm{X}$ & Y & Z \\
\hline 14 & $\mathrm{C}$ & 0.000000 & -1.971081 & -0.808103 \\
\hline 15 & $\mathrm{C}$ & -1.230695 & -2.636655 & -1.038340 \\
\hline 16 & $\mathrm{C}$ & 1.230695 & -2.636655 & -1.038340 \\
\hline 17 & $\mathrm{C}$ & -1.220105 & -3.938844 & -1.514466 \\
\hline 18 & $\mathrm{H}$ & -2.165662 & -2.121272 & -0.844995 \\
\hline 19 & $\mathrm{C}$ & 1.220105 & -3.938844 & -1.514466 \\
\hline 20 & & 62 & -28131272 & -0.844995 \\
\hline 211 & & 0.000000 & -4.587831 & $-d_{4} 751056$ \\
\hline 22 & $\mathrm{H}$ & -2.156285 & -4.454758 & -1.701040 \\
\hline 23 & $\mathrm{H}$ & 2.156285 & -4.454758 & -1.701040 \\
\hline 24 & $\mathrm{H}$ & 0.000000 & -5.609035 & -2.120484 \\
\hline 25 & I & 0.000000 & 0.000000 & 2.090766 \\
\hline & & & & \\
\hline
\end{tabular}




\section{Iodoethyl Cation 19}

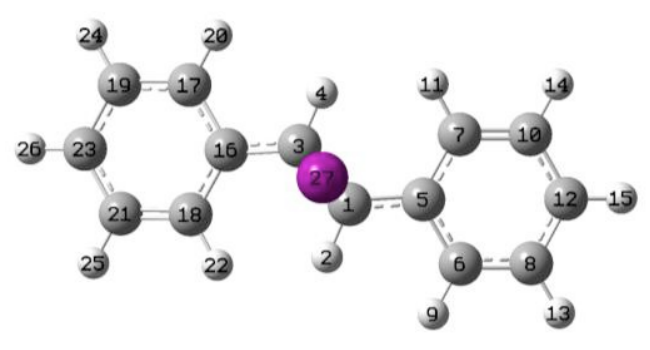

SCF Done: $\quad E(R B 3 L Y P)=-551.823823016$

A.U. after

\begin{tabular}{|c|c|c|c|c|}
\hline No. & Atom & $\mathrm{X}$ & $\mathrm{Y}$ & $\mathrm{Z}$ \\
\hline 1 & $\mathrm{C}$ & -0.550405 & 0.440540 & 0.472650 \\
\hline 2 & $\mathrm{H}$ & -0.279971 & 0.447590 & 1.524321 \\
\hline 3 & $\mathrm{C}$ & 0.550328 & 0.441019 & -0.472826 \\
\hline 4 & $\mathrm{H}$ & 0.279971 & 0.448326 & -1.524519 \\
\hline 5 & $\mathrm{C}$ & -1.909442 & 0.809518 & 0.182489 \\
\hline 6 & $\mathrm{C}$ & -2.398348 & 1.032540 & -1.128846 \\
\hline 7 & $\mathrm{C}$ & -2.784218 & 0.988423 & 1.282785 \\
\hline 8 & $\mathrm{C}$ & -3.711579 & 1.429872 & -1.321691 \\
\hline 9 & $\mathrm{H}$ & -1.756226 & 0.893108 & -1.992569 \\
\hline 10 & $\mathrm{C}$ & -4.097448 & 1.389219 & 1.081732 \\
\hline 11 & $\mathrm{H}$ & -2.418365 & 0.810887 & 2.290540 \\
\hline 12 & $\mathrm{C}$ & -4.562377 & 1.608433 & -0.219846 \\
\hline 13 & $\mathrm{H}$ & -4.082634 & 1.603067 & -2.326617 \\
\hline 14 & $\mathrm{H}$ & -4.759593 & 1.529310 & 1.929844 \\
\hline
\end{tabular}

\begin{tabular}{|c|c|c|c|c|}
\hline No. & Atom & $\mathrm{X}$ & $\mathrm{Y}$ & $\mathrm{Z}$ \\
\hline 15 & $\mathrm{H}$ & -5.590596 & 1.918717 & -0.380273 \\
\hline 16 & $\mathrm{C}$ & 1.909318 & 0.809821 & -0.182523 \\
\hline 17 & $\mathrm{C}$ & 2.784173 & 0.988825 & -1.282752 \\
\hline 18 & $\mathrm{C}$ & 2.398221 & 1.032654 & 1.128895 \\
\hline 19 & $\mathrm{C}$ & 27.097475 & 1.389364 & -1.081594 \\
\hline 20 & $\mathrm{H}$ & 2.418358 & 0.811562 & -2.290570 \\
\hline 21 & $\mathbf{C}$ & 23711482 & 1.429713 & 1.321840 \\
\hline 23252 & $\mathrm{H}$ & 1.755972 & 89.5832998 & 1.992537 \\
\hline 23 & $\mathrm{C}$ & 4.562382 & 1.608261 & 0.220032 \\
\hline 24 & $\mathrm{H}$ & 4.759654 & 1.529502 & -1.929669 \\
\hline $85 \mathrm{cyclesH}$ & 4.082549 & 1.602747 & 2.326788 \\
\hline 26 & $\mathrm{H}$ & 5.590648 & 1.918333 & 0.380573 \\
\hline 27 & $\mathrm{I}$ & -0.000054 & 2.015012 & -0.000046 \\
\hline & & & & \\
\hline
\end{tabular}




\section{Bridged Iodonium Ion 19'}

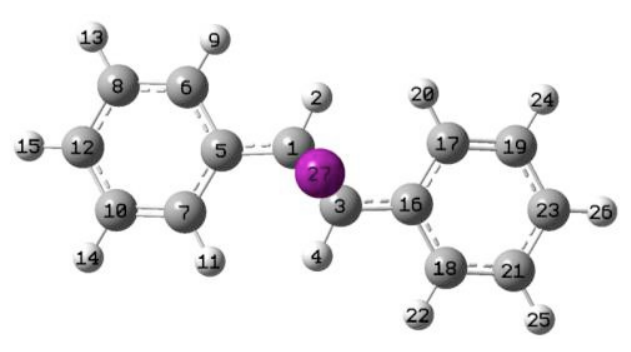

SCF Done: $\quad E(R B 3 L Y P)=-551.823930929$

A.U. after

\begin{tabular}{|c|c|c|c|c|}
\hline No. & Atom & $\mathrm{X}$ & $\mathrm{Y}$ & $\mathrm{Z}$ \\
\hline 1 & $\mathrm{C}$ & 0.000000 & 0.725695 & -0.437179 \\
\hline 2 & $\mathrm{H}$ & 0.973919 & 1.205514 & -0.438917 \\
\hline 3 & $\mathrm{C}$ & 0.000000 & -0.725695 & -0.437179 \\
\hline 4 & $\mathrm{H}$ & -0.973919 & -1.205514 & -0.438917 \\
\hline 5 & $\mathrm{C}$ & -1.103662 & 1.568465 & -0.809302 \\
\hline 6 & $\mathrm{C}$ & -0.842657 & 2.952530 & -0.964956 \\
\hline 7 & $\mathrm{C}$ & -2.410960 & 1.082113 & -1.060591 \\
\hline 8 & $\mathrm{C}$ & -1.848671 & 3.818903 & -1.368139 \\
\hline 9 & $\mathrm{H}$ & 0.155666 & 3.333722 & -0.767296 \\
\hline 10 & $\mathrm{C}$ & -3.410541 & 1.953669 & -1.461834 \\
\hline 11 & $\mathrm{H}$ & -2.643564 & 0.028625 & -0.943851 \\
\hline 12 & $\mathrm{C}$ & -3.132893 & 3.320750 & -1.615525 \\
\hline 13 & $\mathrm{H}$ & -1.639804 & 4.876773 & -1.489183 \\
\hline 14 & $\mathrm{H}$ & -4.409544 & 1.578087 & -1.658041 \\
\hline
\end{tabular}

\begin{tabular}{|c|c|c|c|c|}
\hline No. & Atom & $X$ & Y & $Z$ \\
\hline 15 & $\mathrm{H}$ & -3.922509 & 3.997000 & -1.929305 \\
\hline 16 & $\mathrm{C}$ & 1.103662 & -1.568465 & -0.809302 \\
\hline 17 & $\mathrm{C}$ & 2.410960 & -1.082113 & -1.060591 \\
\hline 18 & $\mathrm{C}$ & 0.842657 & -2.952530 & -0.964956 \\
\hline 19 & $\mathrm{C}$ & $3.4+0541$ & -1.953669 & -1.461834 \\
\hline 20 & $\mathrm{H}$ & 2.643564 & -0.028625 & -0.943851 \\
\hline \multicolumn{4}{|c|}{$213) 6 C_{7}-1.8486716-223.818903$} & -1.368139 \\
\hline 52 & $\mathrm{H}$ & -0.155666 & -3.333722 & -0.767296 \\
\hline 23 & $\mathrm{C}$ & 3.132893 & -3.320750 & -1.615525 \\
\hline 24 & $\mathrm{H}$ & 4.409544 & -1.578087 & -1.658041 \\
\hline$\xi 5^{y}$ & ${ }^{2} \mathrm{H}$ & 1.639804 & -4.876773 & -1.489183 \\
\hline 26 & $\mathrm{H}$ & 3.922509 & -3.997000 & -1.929305 \\
\hline 27 & I & 0.000000 & 0.000000 & 2.020066 \\
\hline
\end{tabular}




\section{Bridged Iodonium Radical 20'}

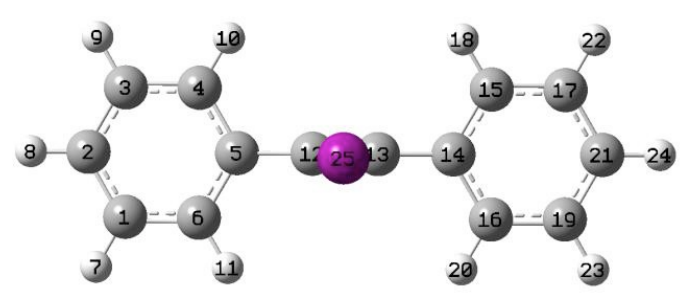

SCF Done: $\quad E(U B 3 L Y P)=-550.822608880$

A.U. after

\begin{tabular}{|c|c|c|c|c|}
\hline No. & Atom & $\mathrm{X}$ & $\mathrm{Y}$ & $\mathrm{Z}$ \\
\hline 14 & $\mathrm{C}$ & 2.028961 & 1.116855 & 0.000000 \\
\hline 15 & $\mathrm{C}$ & 2.737869 & 1.190158 & 1.217011 \\
\hline 16 & $\mathrm{C}$ & 2.737864 & 1.190137 & -1.217015 \\
\hline 17 & $\mathrm{C}$ & 4.121292 & 1.336689 & 1.211033 \\
\hline 18 & $\mathrm{H}$ & 2.192184 & 1.126192 & 2.152898 \\
\hline 19 & $\mathrm{C}$ & 4.121287 & 1.336668 & -1.211046 \\
\hline 20 & $\mathrm{H}$ & 2.1921174 & 1.126155 & -2.152898 \\
\hline $21^{71}$ & $\mathrm{C}$ & 4.816072 & 1.410407 & -0.000008 \\
\hline 22 & $\mathrm{H}$ & 4.660552 & 1.390841 & 2.152452 \\
\hline 23 cycle & 4.660543 & 1.390803 & -2.152467 \\
\hline 24 & $\mathrm{H}$ & 5.896702 & 1.522619 & -0.000011 \\
\hline 25 & $\mathrm{I}$ & -0.000001 & -2.187830 & 0.000006 \\
\hline & & & & \\
\hline
\end{tabular}

\begin{tabular}{|c|c|c|c|c|}
\hline No. & Atom & $\mathrm{X}$ & $\mathrm{Y}$ & $\mathrm{Z}$ \\
\hline 1 & $\mathrm{C}$ & -4.121289 & 1.336664 & -1.211045 \\
\hline 2 & $\mathrm{C}$ & -4.816072 & 1.410407 & -0.000007 \\
\hline 3 & $\mathrm{C}$ & -4.121290 & 1.336694 & 1.211034 \\
\hline 4 & $\mathrm{C}$ & -2.737866 & 1.190165 & 1.217011 \\
\hline 5 & $\mathrm{C}$ & -2.028960 & 1.116857 & -0.000001 \\
\hline 6 & $\mathrm{C}$ & -2.737864 & 1.190135 & -1.217016 \\
\hline 7 & $\mathrm{H}$ & -4.660545 & 1.390795 & -2.152466 \\
\hline 8 & $\mathrm{H}$ & -5.896702 & 1.522618 & -0.000008 \\
\hline 9 & $\mathrm{H}$ & -4.660549 & 1.390849 & 2.152453 \\
\hline 10 & $\mathrm{H}$ & -2.192180 & 1.126203 & 2.152897 \\
\hline 11 & $\mathrm{H}$ & -2.192177 & 1.126150 & -2.152899 \\
\hline 12 & $\mathrm{C}$ & -0.613050 & 0.989230 & -0.000001 \\
\hline 13 & $\mathrm{C}$ & 0.613052 & 0.989225 & 0.000003 \\
\hline
\end{tabular}

